University of South Carolina

Scholar Commons

$1-5-2001$

\title{
An Ellam Scheme for Multidimensional Advection-Reaction Equations and Its Optimal-Order Error Estimate
}

\author{
Hong Wang \\ University of South Carolina - Columbia, wang@math.sc.edu \\ Xiquan Shi \\ Richard E. Ewing
}

Follow this and additional works at: https://scholarcommons.sc.edu/math_facpub

Part of the Mathematics Commons

\section{Publication Info}

Published in Siam Journal of Numerical Analysis, Volume 38, Issue 6, 2001, pages 1846-1885. (C) SIAM Journal On Numerical Analysis 2001, Society for Industrial and Applied Mathematics Wang, H., Shi, X., \& Ewing, R. (2001). An ELLAM Scheme for Multidimensional Advection-Reaction Equations and Its Optimal-Order Error Estimate. SIAM Journal On Numerical Analysis, 38(6), 1846-1885. doi: $10.1137 /$ s0036142999362389

This Article is brought to you by the Mathematics, Department of at Scholar Commons. It has been accepted for inclusion in Faculty Publications by an authorized administrator of Scholar Commons. For more information, please contactdigres@mailbox.sc.edu. 


\title{
AN ELLAM SCHEME FOR MULTIDIMENSIONAL ADVECTION-REACTION EQUATIONS AND ITS OPTIMAL-ORDER ERROR ESTIMATE*
}

\author{
HONG WANG ${ }^{\dagger}$, XIQUAN SHI ${ }^{\ddagger}$, AND RICHARD E. EWING ${ }^{\S}$
}

\begin{abstract}
We present an Eulerian-Lagrangian localized adjoint method (ELLAM) scheme for initial-boundary value problems for advection-reaction partial differential equations in multiple space dimensions. The derived numerical scheme is not subject to the Courant-Friedrichs-Lewy condition and generates accurate numerical solutions even if large time steps are used. Moreover, the scheme naturally incorporates boundary conditions into its formulation without any artificial outflow boundary conditions needed, and it conserves mass. An optimal-order error estimate is proved for the scheme. Numerical experiments are performed to verify the theoretical estimate.
\end{abstract}

Key words. characteristic methods, convergence analysis, error estimates, Eulerian-Lagrangian methods, numerical simulation of advection-reaction equations

AMS subject classifications. 65M25, 65M60, 76M10, 76S05

PII. S0036142999362389

1. Introduction. Advection-dominated reactive transport partial differential equations (PDEs) arise in petroleum reservoir simulation, subsurface contaminant transport, and many other applications and often present serious numerical difficulties $[2,13]$. Space-centered finite difference or finite element methods tend to generate numerical solutions with severe nonphysical oscillations. Upstream weighting techniques are commonly used in industrial applications to stabilize the numerical approximations in most large-scale simulators. However, they produce excessive artificial numerical dispersion, which is of the order of the grid spacing size, and potentially spurious effects related to the orientation of the grid $[13,21]$.

Many specialized schemes have been developed to overcome the difficulties mentioned. Most such methods are based on upstream weighting techniques. The optimal test function methods $[1,5]$ minimize the spatial error and yield an upstream bias in the resulting schemes. The streamline diffusion finite element method [3, 19] adds a numerical diffusion only in the direction of streamlines with no crosswind diffusion introduced. The high resolution methods are well suited for the solution of nonlinear hyperbolic conservation laws and resolve shock discontinuities in the solutions without excessive smearing or spurious oscillations $[7,8,17,24,25,26]$. Because of the hyperbolic nature of advective transport, many characteristic methods have been developed

${ }^{*}$ Received by the editors October 4, 1999; accepted for publication (in revised form) August 16, 2000; published electronically January 5, 2001.

http://www.siam.org/journals/sinum/38-6/36238.html

†Department of Mathematics, University of South Carolina, Columbia, SC 29208 (hwang@ math.sc.edu). This author was supported in part by DOE grant DE-FG05-95ER 25266 and by ONR grant N00014-94-1-1163.

$\ddagger$ Department of Mathematics, Dalian University of Technology, Dalian 116024, China (xqshi@ dlut.edu.cn). This author was supported in part by funding from the Climbing Project Foundation of China, Natural Science Foundation of China, and the Morning-side Mathematics Center of Academia Sinica.

§Institute for Scientific Computation, Texas A\&M University, College Station, TX 77843-3404 (richard-ewing@tamu.edu). This author's work was supported by NSF grants DMS-9626179, DMS9706985, DMS-9707930, NCR9710337, DMS-9972147, and INT-9901498, EPA grant 825207, two generous awards from Mobil Technology Company, and Texas Higher Education Coordinating Board Advanced Research and Technology Program grants 010366-168 and 010366-0336. 
to solve advection-dominated PDEs $[12,16,22,23,27]$. Traditional forward tracking or particle tracking methods [16] advance the grids or the mass associated with the nodes following the characteristics and greatly reduce temporal errors. However, these methods often distort the evolving grids severely or redistribute the mass to the nodes in the future time steps in an ad hoc manner. Other characteristic methods, such as the modified method of characteristics (MMOC) [12], follow the flow by tracking the characteristics backward from a fixed grid at the current time step and, hence, avoid the grid distortion or mass redistribution problems. These methods symmetrize and stabilize the governing equations, greatly reduce temporal errors, thus allowing for large time steps in a simulation without the loss of accuracy, and eliminate the excessive numerical dispersion and grid orientation effects [13]. However, many characteristic methods fail to conserve mass, which is of great concern in virtually all applications.

The difficulties encountered by numerical methods for advection-reaction PDEs are also reflected in their suboptimal-order convergence rates. The linear Galerkin finite element method and upstream weighting method were proven to have a suboptimal-order convergence rate of $\mathcal{O}\left(h+\Delta t\right.$ ) in $L^{2}$ (where $h$ and $\Delta t$ are the sizes of the spatial grids and time steps, respectively) [18]. Despite that characteristic methods have greatly improved accuracy and efficiency, they considerably increase the complexities in their theoretical analyses. The best available estimate for the MMOC (with approximating spaces of piecewise polynomials of degree at most $m$ on a general finite element mesh) for multidimensional linear advection PDEs is only of a suboptimal order $\mathcal{O}\left(h^{m}+\Delta t\right)$ in $L^{2}$, which was proven in [10] under a periodic assumption. It is only in the context of a one-dimensional, constant-coefficient advection equation with periodic data that an optimal-order convergence rate of $\mathcal{O}\left(h^{2}+\Delta t\right)$ in $L^{2}$ was proven for the corresponding MMOC under a fairly restrictive assumption that $\Delta t=\mathcal{O}\left(h^{2}\right)[9]$.

The Eulerian-Lagrangian localized adjoint method (ELLAM) was originally presented in [4] for the solution of one-dimensional, constant-coefficient advection-diffusion PDEs. The ELLAM framework provides a mass-conservative, characteristic solution procedure, and overcomes the principal shortcoming of many characteristic methods while maintaining their numerical advantages. We previously developed an ELLAM scheme for advection-reaction PDEs, which generates a well-conditioned, symmetric and positive-definite coefficient matrix and can be solved efficiently by, for example, the conjugate gradient method in an optimal-order number of iterations without any preconditioning needed. The numerical experiments showed that the ELLAM scheme often outperforms many widely used and well-received methods [28, 29]. Furthermore, in contrast to many previous methods that either impose a periodicity assumption on the advection-reaction PDEs or require that an artificial outflow boundary condition be supplemented, the ELLAM scheme naturally incorporates inflow boundary conditions into its formulation without any artificial outflow boundary conditions needed, and it conserves mass.

The theoretical analysis for ELLAM schemes introduces further difficulties to the already fairly complicated analyses of characteristic methods. These issues include simultaneous a priori estimates for unknowns in interior and at outflow boundaries, and those due to the special treatment of the inflow boundary for mass conservation. Previously, the authors derived an optimal-order error estimate for the ELLAM scheme for the initial-boundary value problems for one-dimensional advection-reaction PDEs $[14,15]$, without requiring the periodic assumption or the restriction that $\Delta t=\mathcal{O}\left(h^{2}\right)$. 
In this paper we derive an optimal order error estimate for the ELLAM scheme for the initial-boundary value problems of multidimensional, advection-reaction PDEs. Because the techniques used in analyzing the one-dimensional ELLAM scheme depend on the Sobolev embedding theorem that the Sobolev space $H^{1}(\Omega) \hookrightarrow C(\bar{\Omega})$ (the space of all continuous functions) which is true only in one space dimension, the analyses in $[14,15]$ would not carry over to multidimensional problems. In this paper we utilize the blending or Boolean interpolation $[11,20]$ and adopt a different approach to derive the error estimate. Then we perform numerical experiments to verify the theoretically proven convergence rates.

The rest of the paper is organized as follows. In section 2, we present an ELLAM scheme. In section 3, we introduce some preliminary notions and blending interpolation estimates. In section 4 , we derive the main result, an optimal-order $L^{2}$ error estimate. In sections 5-8, we prove some lemmas used in section 4 . In section 9 , we perform numerical experiments to verify the theoretical estimates.

2. An ELLAM formulation. We consider the following initial-boundary value problem for the advection-reaction PDE

$$
\begin{aligned}
& u_{t}(\mathbf{x}, t)+\nabla \cdot(\mathbf{v} u(\mathbf{x}, t))+R(\mathbf{x}, t) u=f(\mathbf{x}, t), \quad(\mathbf{x}, t) \in \Omega \times(0, T], \\
& u(\mathbf{x}, t)=g(\mathbf{x}, t), \quad \mathbf{x} \in S^{(I)}, \\
& u(\mathbf{x}, 0)=u_{0}(\mathbf{x}), \quad \mathbf{x} \in \Omega
\end{aligned}
$$

where $\Omega=(a, b) \times(c, d)$ is a rectangular domain with the boundary $\Gamma:=\partial \Omega$. $\mathbf{v}(\mathbf{x}, t):=\left(V_{1}(\mathbf{x}, t), V_{2}(\mathbf{x}, t)\right)$ is a velocity field, $R(\mathbf{x}, t)$ is a first-order reaction coefficient, $f(\mathbf{x}, t)$ is a given function describing source terms, and $u(\mathbf{x}, t)$ is the unknown function representing the solute concentration of a dissolved substance. $S^{(I)}$ is the space-time inflow boundary defined by

$$
S^{(I)}:=\{(\mathbf{x}, t) \in \Gamma \times[0, T] \mid \mathbf{v}(\mathbf{x}, t) \cdot \mathbf{n}(x)<0\}
$$

with $\mathbf{n}(\mathbf{x})$ being the unit outward normal. $g(\mathbf{x}, t)$ and $u_{0}(\mathbf{x})$ are the prescribed inflow boundary and initial conditions, respectively.

2.1. Characteristic tracking and partitions of the domain. Let $N$ be a positive integer. We define a partition of the time interval $[0, T]$ by

$$
t^{n}:=n \Delta t, \quad n=0,1, \ldots, N, \quad \text { with } \Delta t:=\frac{T}{N} .
$$

Multiplying the governing PDE in (2.1) by space-time test functions $w(\mathbf{x}, t)$ that are continuous and piecewise smooth, vanish outside the space-time strip $\Omega \times\left(t^{n}, t^{n+1}\right]$, and are discontinuous in time at time $t^{n}$, we obtain a space-time weak formulation

$$
\begin{aligned}
& \int_{\Omega} u\left(\mathbf{x}, t^{n+1}\right) w\left(\mathbf{x}, t^{n+1}\right) d \mathbf{x}+\int_{t^{n}}^{t^{n+1}} \int_{\Gamma} \mathbf{v} \cdot \mathbf{n}(\mathbf{x}) u(\mathbf{x}, t) w(\mathbf{x}, t) d s d t \\
& -\int_{t^{n}}^{t^{n+1}} \int_{\Omega} u(\mathbf{x}, t)\left(w_{t}+\mathbf{v} \cdot \nabla w-R w\right)(\mathbf{x}, t) d \mathbf{x} d t \\
& =\int_{\Omega} u\left(\mathbf{x}, t^{n}\right) w\left(\mathbf{x}, t_{+}^{n}\right) d \mathbf{x}+\int_{t^{n}}^{t^{n+1}} \int_{\Omega} f(\mathbf{x}, t) w(\mathbf{x}, t) d \mathbf{x} d t
\end{aligned}
$$


where $w\left(\mathbf{x}, t_{+}^{n}\right):=\lim _{t \rightarrow t_{+}^{n}} w(\mathbf{x}, t)$, which takes into account the fact that $w(x, t)$ is discontinuous in time at time $t^{n}$, and

$$
\begin{aligned}
& S_{n}^{(I)}:=\left\{(\mathbf{x}, t) \in \Gamma \times\left[t^{n}, t^{n+1}\right] \mid \mathbf{v}(\mathbf{x}, t) \cdot \mathbf{n}(x)<0\right\}, \\
& S_{n}^{(N)}:=\left\{(\mathbf{x}, t) \in \Gamma \times\left[t^{n}, t^{n+1}\right] \mid \mathbf{v}(\mathbf{x}, t) \cdot \mathbf{n}(x)=0\right\}, \\
& S_{n}^{(O)}:=\left\{(\mathbf{x}, t) \in \Gamma \times\left[t^{n}, t^{n+1}\right] \mid \mathbf{v}(\mathbf{x}, t) \cdot \mathbf{n}(x)>0\right\} .
\end{aligned}
$$

In the ELLAM framework, we choose the test functions $w$ to satisfy the adjoint equation of the governing $\mathrm{PDE}$ in (2.1)

$$
w_{t}+\mathbf{v} \cdot \nabla w-R w=0 .
$$

Equation (2.4) implies that the test functions $w$ should vary exponentially along the characteristics defined by the ordinary differential equation

$$
\frac{d \mathbf{x}}{d \theta}=\mathbf{v}(\mathbf{x}, \theta)
$$

Because one cannot solve (2.5) exactly in practice, one has to use numerical means. For simplicity, we use an Euler quadrature to approximate the characteristics. For any $\mathbf{x} \in \Omega$, we define an approximate characteristic $\mathbf{r}\left(\theta ; \mathbf{x}, t^{n+1}\right)$ emanating backward from $\mathbf{x}$ at time $t^{n+1}$ by

$$
\mathbf{r}\left(\theta ; \mathbf{x}, t^{n+1}\right):=\mathbf{x}-\mathbf{v}\left(\mathbf{x}, t^{n+1}\right)\left(t^{n+1}-\theta\right), \quad \theta \in\left[t^{*}(\mathbf{x}), t^{n+1}\right],
$$

where $t^{*}(\mathbf{x})$ is the time instant when $\mathbf{r}\left(\theta ; \mathbf{x}, t^{n+1}\right)$ backtracks to the boundary $\Gamma$ during the time period $\left[t^{n}, t^{n+1}\right]$, and $t^{*}(\mathbf{x}):=t^{n}$ otherwise.

For any $(\mathbf{x}, t) \in S_{n}^{(O)}$ we define an approximate characteristic $\mathbf{r}(\theta ; \mathbf{x}, t)$ extending backward from $(\mathbf{x}, t)$ by

$$
\mathbf{r}(\theta ; \mathbf{x}, t):=\mathbf{x}-\mathbf{v}(\mathbf{x}, t)(t-\theta), \quad \theta \in\left[t^{*}(\mathbf{x}, t), t\right],
$$

where $t^{*}(\mathbf{x}, t)$ is the time instant when $\mathbf{r}(\theta ; \mathbf{x}, t)$ backtracks to the boundary $\Gamma$ during the time period $\left[t^{n}, t\right]$, and $t^{*}(\mathbf{x}, t):=t^{n}$ otherwise. To accurately measure the effect of the reaction and source terms on a particle traveling from the previous time $t^{n}$ or the inflow boundary $S_{n}^{(I)}$ to the current time $t^{n+1}$ or the outflow boundary $S_{n}^{(O)}$, we define location-dependent time steps $\Delta t^{(I)}(\mathbf{x})=t^{n+1}-t^{*}(\mathbf{x})$ and $\Delta t^{(O)}(\mathbf{x}, t):=t-t^{*}(\mathbf{x}, t)$.

Instead of defining the test functions $w$ to be exponential along the characteristics determined by (2.5), we define the test functions $w$ to be exponential along the approximate characteristics defined by (2.6) and (2.7)

$$
w(\mathbf{r}(\theta ; \overline{\mathbf{x}}, \bar{t}), \theta):=w(\overline{\mathbf{x}}, \bar{t}) e^{-R(\overline{\mathbf{x}}, \bar{t})(\bar{t}-\theta)},
$$

where $\theta \in\left[t^{*}(\mathbf{x}), t^{n+1}\right]$ for $(\overline{\mathbf{x}}, \bar{t})=\left(\mathbf{x}, t^{n+1}\right)$ with $\mathbf{x} \in \Omega$ and $\theta \in\left[t^{*}(\mathbf{x}, t), t\right]$ for $(\overline{\mathbf{x}}, \bar{t})=(\mathbf{x}, t) \in S_{n}^{(O)}$.

2.2. A reference equation. We now evaluate the second term on the righthand side of (2.2). To avoid confusion we replace the dummy variables $\mathbf{x}$ and $t$ in this term by $\mathbf{y}$ and $\theta$, and we reserve $\mathbf{x}$ and $t$ for use in $\Omega$ at time $t^{n+1}$ or at the space-time outflow boundary $S_{n}^{(O)}$. We define

$$
\begin{aligned}
& \Omega^{(O)}(\theta):=\left\{\mathbf{x} \in \Omega \mid \exists \gamma \in\left[\theta, t^{n+1}\right] \text {, such that (s.t.) } \mathbf{r}(\gamma ; \mathbf{x}, \theta) \in \Gamma\right\}, \\
& \Omega^{(I)}(\theta):=\left\{\mathbf{x} \in \Omega \mid \exists(\mathbf{y}, \gamma) \in S_{n}^{(I)} \text { with } \gamma \in\left[t^{n}, \theta\right] \text {, s.t. } \mathbf{x}=\mathbf{r}(\theta ; \mathbf{y}, \gamma)\right\} \text {. }
\end{aligned}
$$


Namely, $\Omega^{(O)}(\theta) \subset \Omega$ is the set of points that will flow out of $\Omega$ during the time period $\left[\theta, t^{n+1}\right]$ and $\Omega^{(I)}(\theta) \subset \Omega$ is the set of points that flowed into $\Omega$ during the time period $\left[t^{n}, \theta\right]$.

For any $\mathbf{y} \in \Omega \backslash \Omega^{(O)}(\theta)$, there exists an $\mathbf{x} \in \Omega$ such that $\mathbf{y}=\mathbf{r}\left(\theta ; \mathbf{x}, t^{n+1}\right)$. Similarly, for any $\mathbf{y} \in \Omega^{(O)}(\theta)$, there exists a pair $(\mathbf{x}, t) \in S_{n}^{(O)}$ such that $\mathbf{y}=\mathbf{r}(\theta ; \mathbf{x}, t)$. Hence

$$
\begin{aligned}
& \int_{t^{n}}^{t^{n+1}} \int_{\Omega^{\prime}} f(\mathbf{y}, \theta) w(\mathbf{y}, \theta) d \mathbf{y} d \theta \\
& =\int_{t^{n}}^{t^{n+1}} \int_{\Omega \backslash \Omega^{(O)}(\theta)} f\left(\mathbf{r}\left(\theta ; \mathbf{x}, t^{n+1}\right), \theta\right) w\left(\mathbf{r}\left(\theta ; \mathbf{x}, t^{n+1}\right), \theta\right) d \mathbf{r} d \theta \\
& \quad+\int_{t^{n}}^{t^{n+1}} \int_{\Omega^{(O)}(\theta)} f(\mathbf{r}(\theta ; \mathbf{x}, t), \theta) w(\mathbf{r}(\theta ; \mathbf{x}, t), \theta) d \mathbf{r} d \theta
\end{aligned}
$$

Enforcing the backward Euler quadrature at $t^{n+1}$ to the first term yields

$$
\begin{aligned}
\int_{t^{n}}^{t^{n+1}} & \int_{\Omega \backslash \Omega^{(O)}(\theta)} f\left(\mathbf{r}\left(\theta ; \mathbf{x}, t^{n+1}\right), \theta\right) w\left(\mathbf{r}\left(\theta ; \mathbf{x}, t^{n+1}\right), \theta\right) d \mathbf{r} d \theta \\
\quad & \int_{\Omega} \int_{t^{*}(\mathbf{x})}^{t^{n+1}} f\left(\mathbf{r}\left(\theta ; \mathbf{x}, t^{n+1}\right), \theta\right) w\left(\mathbf{r}\left(\theta ; \mathbf{x}, t^{n+1}\right), \theta\right) \operatorname{det} \mathbf{J}\left(\theta ; \mathbf{x}, t^{n+1}\right) d \theta d \mathbf{x} \\
& =\int_{\Omega} f\left(\mathbf{x}, t^{n+1}\right) w\left(\mathbf{x}, t^{n+1}\right)\left[\int_{t^{*}(\mathbf{x})}^{t^{n+1}} e^{-R\left(\mathbf{x}, t^{n+1}\right)\left(t^{n+1}-\theta\right)} d \theta\right] d \mathbf{x}+E_{1}(f, w) \\
& =\int_{\Omega} \Psi^{(1)}\left(\mathbf{x}, t^{n+1}\right) f\left(\mathbf{x}, t^{n+1}\right) w\left(\mathbf{x}, t^{n+1}\right) d \mathbf{x}+E_{1}(f, w),
\end{aligned}
$$

where $\operatorname{det} \mathbf{J}\left(\theta ; \mathbf{x}, t^{n+1}\right):=\operatorname{det}\left(\frac{\partial \mathbf{r}\left(\theta ; \mathbf{x}, t^{n+1}\right)}{\partial \mathbf{x}}\right)$ is the Jacobian determinant of the transformation from $\mathbf{r}$ to $\mathbf{x} . \Psi^{(1)}\left(\mathbf{x}, t^{n+1}\right):=\left(1-e^{-R\left(\mathbf{x}, t^{n+1}\right) \Delta t^{(I)}(\mathbf{x})}\right) / R\left(\mathbf{x}, t^{n+1}\right)$ if $R\left(\mathbf{x}, t^{n+1}\right) \neq$ 0 and $\Psi^{(1)}\left(\mathbf{x}, t^{n+1}\right):=\Delta t^{(I)}(\mathbf{x})$ otherwise. $E_{1}(f, w)$ is defined by

$$
\begin{aligned}
E_{1}(f, w):=\int_{\Omega} & \int_{t^{*}(\mathbf{x})}^{t^{n+1}}\left[f\left(\mathbf{r}\left(\theta ; \mathbf{x}, t^{n+1}\right), \theta\right) \operatorname{det} \mathbf{J}\left(\theta ; \mathbf{x}, t^{n+1}\right)-f\left(\mathbf{x}, t^{n+1}\right)\right] \\
& \times w\left(\mathbf{x}, t^{n+1}\right) e^{-R\left(\mathbf{x}, t^{n+1}\right)\left(t^{n+1}-\theta\right)} d \theta d \mathbf{x} .
\end{aligned}
$$

The second term on the right-hand side of (2.10) is written as

$$
\begin{aligned}
& \int_{t^{n}}^{t^{n+1}} \int_{\Omega^{(O)}(\theta)} f(\mathbf{r}(\theta ; \mathbf{x}, t), \theta) w(\mathbf{r}(\theta ; \mathbf{x}, t), \theta) d \mathbf{r} d \theta \\
& =\int_{S_{n}^{(O)}} \int_{t^{*}(\mathbf{x}, t)}^{t} f(\mathbf{r}(\theta ; \mathbf{x}, t), \theta) w(\mathbf{r}(\theta ; \mathbf{x}, t), \theta) \operatorname{det} \mathbf{J}(\theta ; \mathbf{x}, t) \mathbf{v}(\mathbf{x}, t) \cdot \mathbf{n}(\mathbf{x}) d \theta d S \\
& =\int_{S_{n}^{(O)}} \mathbf{v}(\mathbf{x}, t) \cdot \mathbf{n}(\mathbf{x}) f(\mathbf{x}, t) w(\mathbf{x}, t)\left[\int_{t^{*}(\mathbf{x}, t)}^{t} e^{-R(\mathbf{x}, t)(t-\theta)} d \theta\right] d S+E_{2}(f, w) \\
& =\int_{S_{n}^{(O)}} \mathbf{v}(\mathbf{x}, t) \cdot \mathbf{n}(\mathbf{x}) \Psi^{(2)}(\mathbf{x}, t) f(\mathbf{x}, t) w(\mathbf{x}, t) d S+E_{2}(f, w),
\end{aligned}
$$

where $\Psi^{(2)}(\mathbf{x}, t):=\left(1-e^{-R(\mathbf{x}, t) \Delta t^{(O)}(\mathbf{x}, t)}\right) / R(\mathbf{x}, t)$ if $R(\mathbf{x}, t) \neq 0$ and $\Psi^{(2)}(\mathbf{x}, t):=$ $\Delta t^{(O)}(\mathbf{x}, t)$ otherwise. $E_{2}(f, w)$ is defined by

$$
\begin{aligned}
E_{2}(f, w):= & \int_{S_{n}^{(O)}} \int_{t^{*}(\mathbf{x}, t)}^{t} \mathbf{v}(\mathbf{x}, t) \cdot \mathbf{n}(\mathbf{x})[f(\mathbf{r}(\theta ; \mathbf{x}, t), \theta) \operatorname{det} \mathbf{J}(\theta ; \mathbf{x}, t) \\
& -f(\mathbf{x}, t)] \times w(\mathbf{x}, t) e^{-R(\mathbf{x}, t)(t-\theta)} d \theta d S
\end{aligned}
$$


Substituting (2.10), (2.11), and (2.13) for the second term on the right-hand side of (2.2) and incorporating the inflow boundary condition in (2.1) into (2.2), we obtain a weak formulation for the governing PDE in (2.1):

$$
\begin{aligned}
& \int_{\Omega} u\left(\mathbf{x}, t^{n+1}\right) w\left(\mathbf{x}, t^{n+1}\right) d \mathbf{x}+\int_{S_{n}^{(O)}} \mathbf{v}(\mathbf{x}, t) \cdot \mathbf{n}(\mathbf{x}) u(\mathbf{x}, t) w(\mathbf{x}, t) d S \\
& \quad=\int_{\Omega} u\left(\mathbf{x}, t^{n}\right) w\left(\mathbf{x}, t_{+}^{n}\right) d \mathbf{x}+\int_{\Omega} \Psi^{(1)}\left(\mathbf{x}, t^{n+1}\right) f\left(\mathbf{x}, t^{n+1}\right) w\left(\mathbf{x}, t^{n+1}\right) d \mathbf{x} \\
& \quad+\int_{S_{n}^{(O)}} \mathbf{v}(\mathbf{x}, t) \cdot \mathbf{n}(\mathbf{x}) \Psi^{(2)}(\mathbf{x}, t) f(\mathbf{x}, t) w(\mathbf{x}, t) d S \\
& -\int_{S_{n}^{(I)}} \mathbf{v}(\mathbf{x}, t) \cdot \mathbf{n}(\mathbf{x}) g(\mathbf{x}, t) w(\mathbf{x}, t) d S+E(w)
\end{aligned}
$$

with

$$
\begin{aligned}
E(w):= & E_{1}(f, w)+E_{2}(f, w) \\
& +\int_{t^{n}}^{t^{n+1}} \int_{\Omega} u(\mathbf{x}, t)\left(w_{t}+\mathbf{v} \cdot \nabla w-R w\right)(\mathbf{x}, t) d \mathbf{x} d t .
\end{aligned}
$$

2.3. A numerical scheme. We define a uniform rectangular partition on $\Omega$

$$
\begin{array}{ll}
\mathcal{T}^{x}: \quad x_{i}:=a+i \Delta x, \quad i=1,2, \ldots, I, & \text { with } \quad \Delta x:=\frac{b-a}{I}, \\
\mathcal{T}^{y}: & y_{j}:=a+j \Delta y, \quad j=1,2, \ldots, J,
\end{array}
$$

Let $h=\left[(\Delta x)^{2}+(\Delta y)^{2}\right]^{1 / 2}$ be the diameter of the partition; we assume that the partition is quasi-uniform, i.e.,

$$
1 \leq \frac{h}{\min \{\Delta x, \Delta y\}} \leq M_{1}<+\infty
$$

Without loss of generality, we assume that $V_{1}(\mathbf{x}, t)$ and $V_{2}(\mathbf{x}, t)$ are positive on the space-time boundary $\Gamma \times[0, T]$. In this case, the spatial inflow and outflow boundaries $\Gamma^{(I)}$ and $\Gamma^{(O)}$ are independent of time, and the $S_{n}^{(I)}$ and $S_{n}^{(O)}$ defined in $(2.3)$ are reduced to

$$
\begin{aligned}
& \Gamma^{(I)}=\{(a, y) \mid y \in[c, d]\} \cup\{(x, c) \mid x \in[a, b]\}, S_{n}^{(I)}=\Gamma^{(I)} \times\left[t^{n}, t^{n+1}\right] \\
& \Gamma^{(O)}=\{(b, y) \mid y \in[c, d]\} \cup\{(x, d) \mid x \in[a, b]\}, S_{n}^{(O)}=\Gamma^{(O)} \times\left[t^{n}, t^{n+1}\right]
\end{aligned}
$$

Notice that during the time period $\left[t^{n}, t^{n+1}\right]$, the number of spatial degrees of freedom crossing the outflow boundary $S_{n}^{(O)}$ is essentially the Courant number in the normal direction. To preserve the information, we should refine in time at the outflow boundary $S_{n}^{(O)}$ with the same number of degrees of freedom. Let

$$
C r^{(O)}:=\max _{(\mathbf{x}, t) \in S_{n}^{(O)}}\left\{\frac{\left|V_{1}(\mathbf{x}, t)\right| \Delta t}{\Delta x}, \frac{\left|V_{2}(\mathbf{x}, t)\right| \Delta t}{\Delta y}\right\}
$$

be the Courant number with $\left[\mathrm{Cr}^{(\mathrm{O})}\right]$ being its integer part. We define a local refinement in time at the space-time outflow boundary $S_{n}^{(O)}$ by

$$
\mathcal{T}^{t}: \quad t_{n, k}:=t^{n+1}-k \Delta t_{f}, \quad k=0,1, \ldots, I C, \quad \text { with } \Delta t_{f}:=\frac{\Delta t}{I C},
$$


with $I C=\left[C r^{(O)}\right]+1$. The combination of the local refinement $(2.19)$ with the spatial partition $(2.17)$ forms a partition on the space-time boundary $S_{n}^{(O)}$.

Let $\phi(\mathbf{x})$ be any piecewise-bilinear function defined on $\bar{\Omega}$ with the partition $\mathcal{T}^{x} \times \mathcal{T}^{y}$, and let $\phi(\mathbf{x}, t)$ be any piecewise-bilinear function on the space-time outflow boundary $S_{n}^{(O)}$ with the partition $\mathcal{T}^{x} \times \mathcal{T}^{t}$ or $\mathcal{T}^{y} \times \mathcal{T}^{t}$. Then the expression (2.8) leads to the following definitions of the test functions $w(\mathbf{x}, t)$ in the space-time strip $\bar{\Omega} \times\left[t^{n}, t^{n+1}\right]:$

$$
\begin{aligned}
w\left(\mathbf{r}\left(\theta ; \mathbf{x}, t^{n+1}\right), \theta\right) & :=\phi(\mathbf{x}) e^{-R\left(\mathbf{x}, t^{n+1}\right)\left(t^{n+1}-\theta\right)}, \quad \theta \in\left[t^{*}(\mathbf{x}), t^{n+1}\right], \mathbf{x} \in \bar{\Omega} \\
w(\mathbf{r}(\theta ; \mathbf{x}, t), \theta) & :=\phi(\mathbf{x}, t) e^{-R(\mathbf{x}, t)(t-\theta)}, \quad \theta \in\left[t^{*}(\mathbf{x}, t), t\right],(\mathbf{x}, t) \in S_{n}^{(O)}
\end{aligned}
$$

where $t^{*}(\mathbf{x})$ and $t^{*}(\mathbf{x}, t)$ are defined below (2.7).

With the known solution $u\left(\mathbf{x}, t^{n}\right)$ on $\Omega$ at time $t^{n}$ and the inflow boundary condition on $S_{n}^{(I)}$, the weak formulation (2.15) solves for $u\left(\mathbf{x}, t^{n+1}\right)$ on $\Omega$ at time $t^{n+1}$ and $u(\mathbf{x}, t)$ on the space-time outflow boundary $S_{n}^{(O)}$ with no equations being imposed on $S_{n}^{(I)}$ at time $t^{n+1}$ or on $S_{n}^{(O)}$ at time $t^{n}$ [4]. However, to conserve mass, all the test functions in the numerical scheme should sum to one on $\bar{\Omega}$ at time $t^{n+1}$ and on the space-time outflow boundary $S_{n}^{(O)}$. Therefore, we add the basis functions at the nodes on $S_{n}^{(O)}$ at $t^{n}$ to those at their neighboring nodes on $S_{n}^{(O)}$ at $t_{n, I C-1}$, leading to the modified nodal basis functions $\hat{\phi}_{i, J, k}$ and $\hat{\phi}_{I, j, k}$. To maintain the stability and coercivity of the ELLAM scheme, we use these modified basis functions for both trial and test functions. Similarly, we add the basis functions of the test functions at the nodes on $S_{n}^{(I)}$ with $t=t^{n+1}$ to those at their neighboring nodes on $\Omega$ at time $t^{n+1}$. For example, at the nodes $\mathbf{x}_{1, j}(j=1,2, \ldots, J)$ the basis functions $\hat{\phi}_{1, j}(\mathbf{x}):=\phi_{0, j}(\mathbf{x})+\phi_{1, j}(\mathbf{x})$ are constant in $x$ direction on the interval $\left[x_{0}, x_{1}\right]$. At all other nodes that are not adjacent to a node on $S_{n}^{(I)}$ at $t^{n+1}$, the functions $\hat{\phi}_{i, j}=\phi_{i, j}$. One can easily see that the test functions $\hat{w}(\mathbf{x}, t)$ defined this way sum to one on $\Omega$ at time $t^{n+1}$ and on the space-time outflow boundary $S_{n}^{(O)}$. However, to have second-order accuracy in space, we cannot use $\hat{\phi}_{i, j}(\mathbf{x})$ as the same basis functions for the trial functions in $(2.20)$. Instead, we use the standard bilinear functions $\phi_{i, j}(\mathbf{x})$ on $\Omega$ at time $t^{n+1}$ for the trial functions. In summary, the trial functions are defined on $\bar{\Omega}$ at time $t^{n+1}$ and on the space-time outflow boundary $S_{n}^{(O)}$, which have the following expressions under the assumption (2.18):

$$
\begin{aligned}
& U\left(\mathbf{x}, t^{n+1}\right):=\sum_{j=1}^{J} \sum_{i=1}^{I} U\left(\mathbf{x}_{i, j}, t^{n+1}\right) \phi_{i, j}(\mathbf{x})+\sum_{i=0}^{I} g\left(\mathbf{x}_{i, 0}, t^{n+1}\right) \phi_{i, 0}(\mathbf{x}) \\
&+\sum_{j=1}^{J} g\left(\mathbf{x}_{0, j}, t^{n+1}\right) \phi_{0, j}(\mathbf{x}), \quad \mathbf{x} \in \bar{\Omega}, \\
& U(\mathbf{x}, t) \quad:=\sum_{j=0}^{J-1} \sum_{k=0}^{I C-1} U\left(\mathbf{x}_{I, j}, t_{n, k}\right) \hat{\phi}_{I, j, k}(\mathbf{x}, t) \\
&+\sum_{i=0}^{I} \sum_{k=0}^{I C-1} U\left(\mathbf{x}_{i, J}, t_{n, k}\right) \hat{\phi}_{i, J, k}(\mathbf{x}, t), \quad(\mathbf{x}, t) \in S_{n}^{(O)},
\end{aligned}
$$

where $\mathbf{x}_{i, j}:=\left(x_{i}, y_{j}\right) . \quad \phi_{i, j}(\mathbf{x})(0 \leq i \leq I, 0 \leq j \leq J)$ are the nodal basis functions satisfying $\phi_{i, j}\left(\mathbf{x}_{l, m}\right)=\delta_{i, l} \delta_{j, m}$, where $\delta_{i, j}=1$ if $i=j$ and 0 otherwise. $\phi_{I, j, k}(\mathbf{x}, t)(0 \leq j \leq J)$ and $\phi_{i, J, k}(\mathbf{x}, t)(0 \leq i \leq I)$ are the nodal basis functions 
satisfying $\phi_{I, j, k}\left(\mathbf{x}_{I, l}, t_{n, m}\right)=\delta_{j, l} \delta_{k, m}$ and $\phi_{i, J, k}\left(\mathbf{x}_{l, J}, t_{n, m}\right)=\delta_{i, l} \delta_{k, m} \cdot \hat{\phi}_{I, j, k}=\phi_{I, j, k}$ and $\hat{\phi}_{i, J, k}=\phi_{i, J, k}$ for $k=0,1, \ldots, I C-2$, and $\hat{\phi}_{I, j, I C-1}=\phi_{I, j, I C-1}+\phi_{I, j, I C}$ and $\hat{\phi}_{i, J, I C-1}=\phi_{i, J, I C-1}+\phi_{i, J, I C \text {. }}$

Incorporating the trial and test functions into (2.15) and dropping the error term on the right-hand side, we obtain the following ELLAM scheme:

$$
\begin{aligned}
& \int_{\Omega} U\left(\mathbf{x}, t^{n+1}\right) \hat{w}\left(\mathbf{x}, t^{n+1}\right) d \mathbf{x}+\int_{S_{n}^{(O)}} \mathbf{v} \cdot \mathbf{n} U(\mathbf{x}, t) \hat{w}(\mathbf{x}, t) d S \\
& =\int_{\Omega} U\left(\mathbf{x}, t^{n}\right) \hat{w}\left(\mathbf{x}, t_{+}^{n}\right) d \mathbf{x}+\int_{\Omega} \Psi^{(1)}\left(\mathbf{x}, t^{n+1}\right) f\left(\mathbf{x}, t^{n+1}\right) \hat{w}\left(\mathbf{x}, t^{n+1}\right) d \mathbf{x} \\
& +\int_{S_{n}^{(O)}} \Psi^{(2)}(\mathbf{x}, t) \mathbf{v} \cdot \mathbf{n} f(\mathbf{x}, t) \hat{w}(\mathbf{x}, t) d S-\int_{S_{n}^{(I)}} \mathbf{v} \cdot \mathbf{n} g(\mathbf{x}, t) \hat{w}(\mathbf{x}, t) d S
\end{aligned}
$$

where $U(\mathbf{x}, 0)$ has the same form as $(2.20)$ and is defined to be the $L^{2}$ projection of $u_{0}(\mathbf{x})$ given in $(2.1)$.

It is easy to see that the ELLAM scheme (2.21) generates a regularly structured, well-conditioned, symmetric, and positive-definite coefficient matrix. Hence, the resulting algebraic system can be solved efficiently by, for example, the conjugate gradient method in an optimal order number of iterations without any preconditioning needed. Moreover, in contrast to many previous methods which either impose a periodicity assumption on the advection-reaction PDE in (2.1) or require that an artificial outflow boundary condition be supplemented, the ELLAM scheme (2.21) naturally incorporates the inflow boundary condition in (2.1) into its formulation and yields a mass-conservative scheme without any artificial outflow boundary conditions needed. Furthermore, by a judicious choice of the test functions that appear in the weak form (2.2), the relative importance of the advection and reaction components in the governing PDE in (2.1) is directly incorporated into the ELLAM scheme (2.21). We refer readers to $[28,29]$ for more detailed information on the implementational issues of the ELLAM scheme.

\section{Preliminaries and blending interpolation.}

3.1. Preliminary notions. Let $L^{p}(\Omega), 1 \leq p \leq+\infty$, be the standard normed spaces of $p$ th power Lebesgue integrable functions. Then we define the Sobolev spaces

$$
W^{m, p}(\Omega)=\left\{v(\mathbf{x}) \mid \frac{\partial^{i+j} v(x, y)}{\partial x^{i} \partial y^{j}} \in L^{p}(\Omega), \quad 0 \leq i+j \leq m\right\}
$$

with the norms

$$
\|v\|_{W^{m, p}(\Omega)}= \begin{cases}{\left[\sum_{0 \leq i+j \leq m}\left\|\frac{\partial^{i+j} v(x, y)}{\partial x^{i} \partial y^{j}}\right\|_{L^{p}(\Omega)}^{p}\right]^{\frac{1}{p}},} & 1 \leq p<+\infty \\ \max _{0 \leq i+j \leq m}\left\|\frac{\partial^{i+j} v(x, y)}{\partial x^{i} \partial y^{j}}\right\|_{L^{\infty}(\Omega)}, & p=+\infty .\end{cases}
$$

In particular, we let $H^{m}(\Omega)=W^{m, 2}(\Omega)$ and $H^{0}(\Omega)=L^{2}(\Omega)$. In addition, for $1 \leq p, q \leq+\infty$ we define the normed spaces

$$
\begin{aligned}
& L^{q}\left(t_{1}, t_{2} ; W^{m, p}(\Omega)\right) \\
& \quad=\left\{w(\mathbf{x}, t) \mid w(\cdot, t):\left(t_{1}, t_{2}\right) \longmapsto W^{m, p}(\Omega), \quad\|w(\cdot, t)\|_{W^{m, p}(\Omega)} \in L^{q}\left(t_{1}, t_{2}\right)\right\}
\end{aligned}
$$


with the norms

$$
\begin{aligned}
\|w\|_{L^{q}\left(t_{1}, t_{2} ; W^{m, p}(\Omega)\right)} & =\|\| w(\cdot, t)\left\|_{W^{m, p}(\Omega)}\right\|_{L^{q}\left(t_{1}, t_{2}\right)}, \quad 1 \leq p, q \leq+\infty \\
\|w\|_{\hat{L}^{\infty}\left(0, T ; L^{2}(\Omega)\right)} & =\max _{0 \leq n \leq N}\left\|w\left(\cdot, t^{n}\right)\right\|_{L^{2}(\Omega)}
\end{aligned}
$$

Let $p(x) \in C[a, b]$, the space of continuous functions on $[a, b]$, and $\mathcal{T}^{x}$ be a partition on $[a, b]$ given in (2.17). We define $\Pi^{x}$ to be an interpolation operator from $C[a, b]$ to the space of piecewise-linear functions on $[a, b]$ with the partition $\mathcal{T}^{x}$ :

$$
\left.\left(\Pi^{x} p\right)(x)\right|_{\left[x_{i-1}, x_{i}\right]}:=p\left(x_{i-1}\right) \phi_{i}^{(1)}(x)+p\left(x_{i}\right) \phi_{i}^{(2)}(x), \quad 1 \leq i \leq I,
$$

with

$$
\phi_{i}^{(1)}(x):=\frac{x_{i}-x}{\Delta x}, \quad \phi_{i}^{(2)}(x):=\frac{x-x_{i-1}}{\Delta x}, \quad x \in\left[x_{i-1}, x_{i}\right], \quad 1 \leq i \leq I .
$$

Similarly, for any $q(x, y) \in C(\bar{\Omega})$, the space of continuous functions on $\bar{\Omega}$, we define $\Pi^{\mathrm{I}}$ to be an interpolation operator from $C(\bar{\Omega})$ to $Q_{h}(\bar{\Omega})$, the space of piecewisebilinear functions on $\bar{\Omega}$ with the partition $\mathcal{T}^{x} \times \mathcal{T}^{y}$ defined in (2.17):

$$
\begin{aligned}
\left.\left(\Pi^{\mathrm{I}} q\right)(x, y)\right|_{\bar{\Omega}_{i, j}} & :=\left(\Pi^{x} \Pi^{y} q\right)(x, y)=\left(\Pi^{y} \Pi^{x} q\right)(x, y) \\
& =\sum_{l, m=1}^{2} q\left(x_{i+l-2}, y_{j+m-2}\right) \phi_{i, j}^{(l, m)}(x, y), \quad 1 \leq i \leq I, \quad 1 \leq j \leq J
\end{aligned}
$$

where

$$
\begin{aligned}
\phi_{i, j}^{(l, m)}(x, y) & :=\phi_{i}^{(l)}(x) \phi_{j}^{(m)}(y), \quad(x, y) \in \bar{\Omega}_{i, j}:=\left[x_{i-1}, x_{i}\right] \times\left[y_{j-1}, y_{j}\right] \\
& 1 \leq l, m \leq 2, \quad 1 \leq i \leq I, \quad 1 \leq j \leq J
\end{aligned}
$$

It is well known that the following estimates hold [6]:

$$
\begin{aligned}
\left\|\Pi^{\mathrm{I}} q-q\right\|_{W^{m, p}(\Omega)} \leq M h^{2-m}\|q\|_{W^{2, p}(\Omega)} & \forall q \in H^{2}(\Omega), \quad m=0,1 \\
\|q\|_{W^{m, \infty}(\Omega)} \leq M h^{-1}\|q\|_{H^{m}(\Omega)} & \forall q \in Q_{h}(\bar{\Omega}), \quad m=0,1 .
\end{aligned}
$$

Although the estimate in (3.2) is of optimal-order, it is not refined enough. We need a more refined expansion in our analysis. For example, it is well known that in the one-dimensional case, the following error expansions hold:

$$
\begin{aligned}
\left(\Pi^{x} p\right)(x)-\left.p(x)\right|_{\left[x_{i-1}, x_{i}\right]} & =\int_{x_{i-1}}^{x_{i}} K_{i}^{(m)}(\alpha ; x) \frac{d^{m} p(\alpha)}{d^{m} \alpha} d \alpha, \quad 1 \leq i \leq I \\
m & =1,2
\end{aligned}
$$

where the Kernels $K_{i}^{(1)}(\alpha ; x)$ and $K_{i}^{(2)}(\alpha ; x)$ are defined by

$$
K_{i}^{(1)}(\alpha ; x)=\left\{\begin{aligned}
-\phi_{i}^{(1)}(x) & =-\frac{x_{i}-x}{\Delta x}, & & \alpha \in\left[x_{i-1}, x\right] \\
\phi_{i}^{(2)}(x) & =\frac{x-x_{i-1}}{\Delta x}, & & \alpha \in\left[x, x_{i}\right]
\end{aligned}\right.
$$

and

$$
K_{i}^{(2)}(\alpha ; x)= \begin{cases}\phi_{i}^{(1)}(x)\left(\alpha-x_{i-1}\right)=\frac{\left(x_{i}-x\right)\left(\alpha-x_{i-1}\right)}{\Delta x}, & \alpha \in\left[x_{i-1}, x\right], \\ \phi_{i}^{(2)}(x)\left(x_{i}-\alpha\right)=\frac{\left(x-x_{i-1}\right)\left(x_{i}-\alpha\right)}{\Delta x}, & \alpha \in\left[x, x_{i}\right] .\end{cases}
$$

Equation (3.3) holds for $p \in H^{m}(a, b)$ with $m=1$ or 2 . 
3.2. Blending interpolation. To derive an analogous expansion for the error of bilinear interpolation $\left(\Pi^{\mathrm{I}} q\right)(x, y)-q(x, y)$, we utilize the blending or Boolean interpolation $\left(\Pi^{\mathrm{B}} q\right)(x, y)[11,20]$ defined by

$$
\begin{aligned}
\left(\Pi^{\mathrm{B}} q\right) & \left.(x, y)\right|_{\bar{\Omega}_{i, j}}:=\left(\Pi^{x}+\Pi^{y}-\Pi^{x} \Pi^{y} q\right)(x, y) \\
& =\sum_{l=1}^{2}\left[q\left(x_{i+l-2}, y\right) \phi_{i}^{(l)}(x)+q\left(x, y_{j+l-2}\right) \phi_{j}^{(l)}(y)\right] \\
& -\sum_{l, m=1}^{2} q\left(x_{i+l-2}, y_{j+m-2}\right) \phi_{i, j}^{(l, m)}(x, y), \quad 1 \leq i \leq I, 1 \leq j \leq J .
\end{aligned}
$$

Using (3.3), we obtain the following error expansions for the blending interpolant:

$$
\begin{gathered}
\left(\Pi^{\mathrm{B}} q\right)(x, y)-\left.q(x, y)\right|_{\bar{\Omega}_{i, j}}=-\left.\left(\Pi^{y}-\mathrm{I}\right)\left(\Pi^{x}-\mathrm{I}\right) q(x, y)\right|_{\bar{\Omega}_{i, j}} \\
=-\left(\Pi^{y}-\mathrm{I}\right) \int_{x_{i-1}}^{x_{i}} K_{i}^{(l)}(\alpha ; x) \frac{\partial^{l} q}{\partial \alpha^{l}}(\alpha, y) d \alpha \\
=-\int_{y_{j-1}}^{y_{j}} \int_{x_{i-1}}^{x_{i}} K_{i, j}^{(l, m)}(\alpha, \beta ; x, y) \frac{\partial^{l+m} q}{\partial \alpha^{l} \beta^{m}}(\alpha, \beta) d \alpha d \beta, \\
1 \leq i \leq I, \quad 1 \leq j \leq J, \quad 1 \leq l, m \leq 2,
\end{gathered}
$$

with

$$
K_{i, j}^{(l, m)}(\alpha, \beta ; x, y)=K_{i}^{(l)}(\alpha ; x) K_{j}^{(m)}(\beta ; y) .
$$

Equation (3.6) holds for $q \in H^{l+m}(\Omega)$ with $1 \leq l, m \leq 2$.

Using (3.3), we obtain an expansion for $\left(\bar{\Pi}^{\mathrm{I}}-\Pi^{\mathrm{B}}\right) q(x, y)$ :

$$
\begin{aligned}
\left.\left(\Pi^{\mathrm{I}}-\Pi^{\mathrm{B}}\right) q(x, y)\right|_{\Omega_{i, j}} & {\left.\left[\Pi^{y}\left(\Pi^{x}-\mathrm{I}\right)+\Pi^{x}\left(\Pi^{y}-\mathrm{I}\right)\right] q(x, y)\right|_{\bar{\Omega}_{i, j}} } \\
= & \Pi^{y} \int_{x_{i-1}}^{x_{i}} K_{i}^{(2)}(\alpha ; x) q_{\alpha \alpha}(\alpha, y) d \alpha+\Pi^{x} \int_{y_{j-1}}^{y_{j}} K_{j}^{(2)}(\beta ; y) q_{\beta \beta}(x, \beta) d \beta \\
= & \int_{x_{i-1}}^{x_{i}} K_{i}^{(2)}(\alpha ; x) \Pi^{y} q_{\alpha \alpha}(\alpha, y) d \alpha+\int_{y_{j-1}}^{y_{j}} K_{j}^{(2)}(\beta ; y) \Pi^{x} q_{\beta \beta}(x, \beta) d \beta \\
= & \int_{x_{i-1}}^{x_{i}} K_{i}^{(2)}(\alpha ; x) q_{\alpha \alpha}(\alpha, y) d \alpha+\int_{y_{j-1}}^{y_{j}} K_{j}^{(2)}(\beta ; y) q_{\beta \beta}(x, \beta) d \beta \\
& +\int_{y_{j-1}}^{y_{j}} \int_{x_{i-1}}^{x_{i}} K_{i, j}^{(2,1)}(\alpha, \beta ; x, y) q_{\alpha \alpha \beta}(\alpha, \beta) d \alpha d \beta \\
& +\int_{y_{j-1}}^{y_{j}} \int_{x_{i-1}}^{x_{i}} K_{i, j}^{(1,2)}(\alpha, \beta ; x, y) q_{\alpha \beta \beta}(\alpha, \beta) d \alpha d \beta, \quad 1 \leq i \leq I, \quad 1 \leq j \leq J .
\end{aligned}
$$

Using (3.6) and (3.8), we obtain an error expansion for $\left(\Pi^{\mathrm{I}}-\mathrm{I}\right) q$ :

$$
\begin{aligned}
\left.\left(\Pi^{\mathrm{I}}-\mathrm{I}\right) q(x, y)\right|_{\bar{\Omega}_{i, j}}=\left.\left(\Pi^{\mathrm{I}}-\Pi^{\mathrm{B}}\right) q(x, y)\right|_{\bar{\Omega}_{i, j}}+\left.\left(\Pi^{\mathrm{B}}-\mathrm{I}\right) q(x, y)\right|_{\bar{\Omega}_{i, j}} \\
\quad=\int_{x_{i-1}}^{x_{i}} K_{i}^{(2)}(\alpha ; x) q_{\alpha \alpha}(\alpha, y) d \alpha+\int_{y_{j-1}}^{y_{j}} K_{j}^{(2)}(\beta ; y) q_{\beta \beta}(x, \beta) d \beta \\
+\int_{y_{j-1}}^{y_{j}} \int_{x_{i-1}}^{x_{i}} K_{i, j}^{(m, 3-m)}(\alpha, \beta ; x, y) \frac{\partial^{3} q}{\partial \alpha^{m} \beta^{3-m}}(\alpha, \beta) d \alpha d \beta, \\
\quad 1 \leq i \leq I, \quad 1 \leq j \leq J, \quad m=1,2 .
\end{aligned}
$$


3.3. Notations. To derive an optimal-order error estimate for the global truncation errors

$$
\begin{array}{llll}
e\left(\mathbf{x}, t^{n}\right) & :=U\left(\mathbf{x}, t^{n}\right)-u\left(\mathbf{x}, t^{n}\right), & \mathbf{x} \in \bar{\Omega}, & n=0,1, \ldots, N, \\
e(\mathbf{x}, t) & :=U(\mathbf{x}, t)-u(\mathbf{x}, t), & (\mathbf{x}, t) \in S_{n}^{(O)}, & n=0,1, \ldots, N-1,
\end{array}
$$

we introduce the following auxiliary functions in light of the definition of the trial functions $U$ in (2.20):

$$
\begin{aligned}
\Pi^{\mathrm{I}} u\left(\mathbf{x}, t^{n}\right) & :=\sum_{j=1}^{J} \sum_{i=1}^{I} u\left(\mathbf{x}_{i, j}, t^{n}\right) \phi_{i, j}(\mathbf{x})+\sum_{i=0}^{I} g\left(\mathbf{x}_{i, 0}, t^{n}\right) \phi_{i, 0}(\mathbf{x}) \\
& +\sum_{j=1}^{J} g\left(\mathbf{x}_{0, j}, t^{n}\right) \phi_{0, j}(\mathbf{x}), \quad \mathbf{x} \in \bar{\Omega}, \quad n=0,1, \ldots, N, \\
\Pi^{\mathrm{I}} u(\mathbf{x}, t) \quad:= & \sum_{j=0}^{J-1} \sum_{k=0}^{I C-1} u\left(\mathbf{x}_{I, j}, t_{n, k}\right) \hat{\phi}_{I, j, k}(\mathbf{x}, t) \\
& +\sum_{i=0}^{I} \sum_{k=0}^{I C-1} u\left(\mathbf{x}_{i, J}, t_{n, k}\right) \hat{\phi}_{i, J, k}(\mathbf{x}, t), \\
& (\mathbf{x}, t) \in S_{n}^{(O)}, \quad n=0,1, \ldots, N-1 .
\end{aligned}
$$

Then we can decompose the global truncation errors $e\left(\mathbf{x}, t^{n}\right)$ for $\mathbf{x} \in \bar{\Omega}$ and $e(\mathbf{x}, t)$ for $(\mathbf{x}, t) \in S_{n}^{(O)}$ as follows:

$$
\begin{array}{rlrl}
e\left(\mathbf{x}, t^{n}\right) & =\xi\left(\mathbf{x}, t^{n}\right)-\eta\left(\mathbf{x}, t^{n}\right), & \mathbf{x} \in \bar{\Omega}, & n=0,1, \ldots, N, \\
e(\mathbf{x}, t)=\xi(\mathbf{x}, t)-\eta(\mathbf{x}, t), & (\mathbf{x}, t) \in S_{n}^{(O)}, & n=0,1, \ldots, N-1,
\end{array}
$$

with

$$
\begin{aligned}
& \xi\left(\mathbf{x}, t^{n}\right):=U\left(\mathbf{x}, t^{n}\right)-\Pi^{\mathrm{I}} u\left(\mathbf{x}, t^{n}\right), \quad \xi(\mathbf{x}, t):=U(\mathbf{x}, t)-\Pi^{\mathrm{I}} u(\mathbf{x}, t), \\
& \eta\left(\mathbf{x}, t^{n}\right):=\Pi^{\mathrm{I}} u\left(\mathbf{x}, t^{n}\right)-u\left(\mathbf{x}, t^{n}\right), \quad \eta(\mathbf{x}, t):=\Pi^{\mathrm{I}} u(\mathbf{x}, t)-u(\mathbf{x}, t), \\
& \mathbf{x} \in \bar{\Omega}, \quad n=0,1, \ldots, N, \quad(\mathbf{x}, t) \in S_{n}^{(O)}, \quad n=0,1, \ldots, N-1 .
\end{aligned}
$$

Notice that the error estimates for $\eta\left(\mathbf{x}, t^{n}\right)$ and $\eta(\mathbf{x}, t)$ are known from (3.2), (3.3), and (3.9). Our main objective is to derive an optimal-order error estimate for $\xi\left(\mathbf{x}, t^{n}\right)$ and $\xi(\mathbf{x}, t)$. From definitions $(2.20)$ and (3.10), we obtain the following expressions for $\xi\left(\mathbf{x}, t^{n}\right)$ and $\xi(\mathbf{x}, t)$ :

$$
\begin{aligned}
\xi\left(\mathbf{x}, t^{n}\right)= & \sum_{j=1}^{J} \sum_{i=1}^{I} \xi\left(\mathbf{x}_{i, j}, t^{n}\right) \phi_{i, j}(\mathbf{x}), \quad \mathbf{x} \in \bar{\Omega}, \quad n=0,1, \ldots, N \\
\xi(\mathbf{x}, t)= & \sum_{j=0}^{J-1} \sum_{k=0}^{I C-1} \xi\left(\mathbf{x}_{I, j}, t_{n, k}\right) \hat{\phi}_{I, j, k}(\mathbf{x}, t) \\
& +\sum_{i=0}^{I} \sum_{k=0}^{I C-1} \xi\left(\mathbf{x}_{i, J}, t_{n, k}\right) \hat{\phi}_{i, J, k}(\mathbf{x}, t) \\
& (\mathbf{x}, t) \in S_{n}^{(O)}, \quad n=0,1, \ldots, N-1 .
\end{aligned}
$$


We also introduce the following notation for $\mathbf{x} \in \bar{\Omega}$ and $n=0,1, \ldots, N$ :

$$
\begin{aligned}
\hat{\xi}\left(\mathbf{x}, t^{n}\right) & :=\sum_{j=1}^{J} \sum_{i=1}^{I} \xi\left(\mathbf{x}_{i, j}, t^{n}\right) \phi_{i, j}(\mathbf{x})+\sum_{i=1}^{I} \xi\left(\mathbf{x}_{i, 1}, t^{n}\right) \phi_{i, 0}(\mathbf{x}) \\
& +\sum_{j=1}^{J} \xi\left(\mathbf{x}_{1, j}, t^{n}\right) \phi_{0, j}(\mathbf{x})+\xi\left(\mathbf{x}_{1,1}, t^{n}\right) \phi_{0,0}(\mathbf{x}) .
\end{aligned}
$$

The following expressions, which come from (3.11)-(3.12), will be used frequently in our theoretical analysis:

$$
\begin{aligned}
\xi\left(\mathbf{x}, t^{n}\right) & =\eta\left(\mathbf{x}, t^{n}\right)=0, & & \mathbf{x} \in \Gamma^{(I)}, 0 \leq n \leq N \\
\hat{\xi}\left(\mathbf{x}, t^{n}\right) & =\xi\left(x_{1}, y, t^{n}\right), & & (x, y) \in\left[a, x_{1}\right] \times\left[y_{1}, d\right], n=0,1, \ldots, N \\
\hat{\xi}\left(\mathbf{x}, t^{n}\right) & =\xi\left(x, y_{1}, t^{n}\right), & & (x, y) \in\left[x_{1}, b\right] \times\left[c, y_{1}\right], n=0,1, \ldots, N \\
\hat{\xi}\left(\mathbf{x}, t^{n}\right) & =\xi\left(x_{1}, y_{1}, t^{n}\right), & & (x, y) \in\left[a, x_{1}\right] \times\left[c, y_{1}\right], n=0,1, \ldots, N \\
\xi(\mathbf{x}, t) & =\xi\left(\mathbf{x}, t_{n, I C-1}\right), & & \mathbf{x} \in \Gamma^{(O)}, t \in\left[t^{n}, t_{n, I C-1}\right], 0 \leq n \leq N-1 .
\end{aligned}
$$

We use $\varepsilon$ to denote an arbitrarily small positive number and $M$ to denote a generic positive constant, which may assume different values at different places.

4. Main results. In this section, we derive an optimal-order $L^{2}$ error estimate for the ELLAM scheme (2.21). Subtracting (2.21) from (2.15) we obtain

$$
\begin{aligned}
& \int_{\Omega} e\left(\mathbf{x}, t^{n+1}\right) \hat{\xi}\left(\mathbf{x}, t^{n+1}\right) d \mathbf{x}+\int_{S_{n}^{(O)}} \mathbf{v}(\mathbf{x}, t) \cdot \mathbf{n}(\mathbf{x}) e(\mathbf{x}, t) \xi(\mathbf{x}, t) d S \\
& =\int_{\Omega} e\left(\mathbf{x}, t^{n}\right) \hat{\xi}\left(\mathbf{x}, t_{+}^{n}\right) d \mathbf{x}-E(\hat{\xi})
\end{aligned}
$$

Then the error equation above is rewritten in terms of $\xi$ and $\eta$ as follows:

$$
\begin{aligned}
& \int_{\Omega} \xi\left(\mathbf{x}, t^{n+1}\right) \hat{\xi}\left(\mathbf{x}, t^{n+1}\right) d \mathbf{x}+\int_{S_{n}^{(O)}} \mathbf{v}(\mathbf{x}, t) \cdot \mathbf{n}(\mathbf{x}) \xi^{2}(\mathbf{x}, t) d S \\
(4.1)= & \int_{\Omega} \xi\left(\mathbf{x}, t^{n}\right) \hat{\xi}\left(\mathbf{x}, t_{+}^{n}\right) d \mathbf{x}+\int_{\Omega} \eta\left(\mathbf{x}, t^{n}\right) \hat{\xi}\left(\mathbf{x}, t_{+}^{n}\right) d \mathbf{x} \\
& -\int_{\Omega} \eta\left(\mathbf{x}, t^{n+1}\right) \hat{\xi}\left(\mathbf{x}, t^{n+1}\right) d \mathbf{x}-\int_{S_{n}^{(O)}} \mathbf{v}(\mathbf{x}, t) \cdot \mathbf{n}(\mathbf{x}) \eta(\mathbf{x}, t) \xi(\mathbf{x}, t) d S-E(\hat{\xi}) .
\end{aligned}
$$

Using (3.13) we rewrite the first term on the right-hand side of (4.1) as

$$
\begin{aligned}
& \int_{\Omega} \xi\left(\mathbf{x}, t^{n+1}\right) \hat{\xi}\left(\mathbf{x}, t^{n+1}\right) d \mathbf{x} \\
& =\int_{y_{1}}^{d} \int_{x_{1}}^{b} \xi^{2}\left(\mathbf{x}, t^{n+1}\right) d \mathbf{x}+\int_{y_{1}}^{d} \int_{a}^{x_{1}} \xi\left(\mathbf{x}, t^{n+1}\right) \xi\left(x_{1}, y, t^{n+1}\right) d \mathbf{x} \\
(4.2)+ & \int_{c}^{y_{1}} \int_{x_{1}}^{b} \xi\left(\mathbf{x}, t^{n+1}\right) \xi\left(x, y_{1}, t^{n+1}\right) d \mathbf{x}+\int_{c}^{y_{1}} \int_{a}^{x_{1}} \xi\left(\mathbf{x}, t^{n+1}\right) \xi\left(x_{1}, y_{1}, t^{n+1}\right) d \mathbf{x} \\
& =\int_{y_{1}}^{d} \int_{x_{1}}^{b} \xi^{2}\left(\mathbf{x}, t^{n+1}\right) d \mathbf{x}+\frac{\Delta x}{2} \int_{y_{1}}^{d} \xi^{2}\left(x_{1}, y, t^{n+1}\right) d y \\
& +\frac{\Delta y}{2} \int_{x_{1}}^{b} \xi^{2}\left(x, y_{1}, t^{n+1}\right) d x+\frac{\Delta x \Delta y}{4} \xi^{2}\left(\mathbf{x}_{1,1}, t^{n+1}\right) .
\end{aligned}
$$


We now turn to the right-hand side of (4.1). The first term is decomposed as

$$
\begin{aligned}
& \quad \int_{\Omega} \xi\left(\mathbf{y}, t^{n}\right) \hat{\xi}\left(\mathbf{y}, t_{+}^{n}\right) d \mathbf{y} \\
& =\int_{\Omega_{\backslash \Omega^{(O)}\left(t^{n}\right)}} \xi\left(\mathbf{y}, t^{n}\right) \hat{\xi}\left(\mathbf{y}, t_{+}^{n}\right) d \mathbf{y}+\int_{\Omega_{n}^{(O)}} \xi\left(\mathbf{y}, t^{n}\right) \xi\left(\mathbf{y}, t_{+}^{n}\right) d \mathbf{y} \\
& =\int_{\Omega_{\backslash \Omega^{(I)}\left(t^{n+1}\right)}} \xi\left(\mathbf{x}^{*}, t^{n}\right) \hat{\xi}\left(\mathbf{x}, t^{n+1}\right) \operatorname{det} \mathbf{J}\left(t^{n} ; \mathbf{x}, t^{n+1}\right) e^{-R\left(\mathbf{x}, t^{n+1}\right) \Delta t} d \mathbf{x} \\
& \quad+\int_{\Omega_{n}^{(O)}} \xi\left(\mathbf{y}, t^{n}\right) \xi\left(\mathbf{y}, t_{+}^{n}\right) d \mathbf{y} \\
& =\int_{\Omega \backslash \Omega^{(I)}\left(t^{n+1}\right)} \xi\left(\mathbf{x}^{*}, t^{n}\right) \hat{\xi}\left(\mathbf{x}, t^{n+1}\right)\left(\operatorname{det} \mathbf{J}\left(t^{n} ; \mathbf{x}, t^{n+1}\right) e^{-R\left(\mathbf{x}, t^{n+1}\right) \Delta t}-1\right) d \mathbf{x} \\
& +\int_{\Omega \backslash \Omega^{(I)}\left(t^{n+1}\right)} \xi\left(\mathbf{x}^{*}, t^{n}\right) \hat{\xi}\left(\mathbf{x}, t^{n+1}\right) d \mathbf{x}+\int_{\Omega_{n}^{(O)}} \xi\left(\mathbf{y}, t^{n}\right) \xi\left(\mathbf{y}, t_{+}^{n}\right) d \mathbf{y},
\end{aligned}
$$

where $\Omega^{(I)}(\theta)$ and $\Omega^{(O)}(\theta)$ are defined in $(2.9)$.

The first and third terms on the right-hand side of (4.3) are bounded by

$$
\begin{aligned}
& \mid \int_{\Omega \backslash \Omega^{(I)}\left(t^{n+1}\right)} \xi\left(\mathbf{x}^{*}, t^{n}\right) \hat{\xi}\left(\mathbf{x}, t^{n+1}\right)\left(\operatorname{det} \mathbf{J}\left(t^{n} ; \mathbf{x}, t^{n+1}\right) e^{-R\left(\mathbf{x}, t^{n+1}\right) \Delta t}-1\right) d \mathbf{x} \\
& +\int_{\Omega_{n}^{(O)}} \xi\left(\mathbf{y}, t^{n}\right) \xi\left(\mathbf{y}, t_{+}^{n}\right) d \mathbf{y} \mid \\
& \leq \frac{1}{2} \int_{\Omega_{n}^{(O)}} \xi^{2}\left(\mathbf{y}, t_{+}^{n}\right) d \mathbf{y}+\frac{1}{2} \int_{\Omega_{n}^{(O)}} \xi^{2}\left(\mathbf{y}, t^{n}\right) d \mathbf{y} \\
& +M \Delta t \int_{\Omega \backslash \Omega^{(I)}\left(t^{n+1}\right)}\left|\xi\left(\mathbf{x}^{*}, t^{n}\right) \hat{\xi}\left(\mathbf{x}, t^{n+1}\right)\right| d \mathbf{x} \\
& \leq \frac{1+M \Delta t}{2} \int_{S_{n}^{(O)}} \mathbf{v}(\mathbf{x}, t) \cdot \mathbf{n}(\mathbf{x}) \xi^{2}(\mathbf{x}, t) d S+\frac{1}{2} \int_{\Omega_{n}^{(O)}} \xi^{2}\left(\mathbf{y}, t^{n}\right) d \mathbf{y} \\
& +M \Delta t\left\|\xi\left(\mathbf{x}, t^{n+1}\right)\right\|_{L^{2}(\Omega)}^{2}+M \Delta t\left\|\xi\left(\mathbf{x}, t^{n}\right)\right\|_{L^{2}(\Omega)}^{2}
\end{aligned}
$$

However, the estimate for the second term on the right-hand side of (4.3) is very delicate and is derived in Lemma 1 in section 5 . We present only the result here:

$$
\begin{aligned}
(4.4) & +\frac{\Delta x}{4} \int_{y_{1}}^{d} K^{(3)}\left(x_{1}, y\right) \xi^{2}\left(x_{1}, y, t^{n+1}\right) d y+\frac{\Delta y}{4} \int_{x_{1}}^{b} K^{(4)}\left(x, y_{1}\right) \xi^{2}\left(x, y_{1}, t^{n+1}\right) d x \\
& +\frac{\Delta x}{4} \int_{y_{1}}^{d^{*}\left(x_{1}, d\right)} \xi^{2}\left(x_{1}, y, t^{n}\right) d y+\frac{\Delta y}{4} \int_{x_{1}}^{b^{*}\left(b, y_{1}\right)} \xi^{2}\left(x, y_{1}, t^{n}\right) d x \\
& +\frac{\Delta x \Delta y}{8} K^{(3)}\left(\mathbf{x}_{1,1}\right) K^{(4)}\left(\mathbf{x}_{1,1}\right) \xi^{2}\left(\mathbf{x}_{1,1}, t^{n+1}\right)+\frac{\Delta x \Delta y}{8} \xi^{2}\left(\mathbf{x}_{1,1}, t^{n}\right) \\
& +M \Delta t\left\|\xi\left(\mathbf{x}, t^{n+1}\right)\right\|_{L^{2}(\Omega)}^{2}+M \Delta t\left\|\xi\left(\mathbf{x}, t^{n}\right)\right\|_{L^{2}(\Omega)}^{2},
\end{aligned}
$$$$
\left|\int_{\Omega \backslash \Omega^{(I)}\left(t^{n+1}\right)} \xi\left(\mathbf{x}^{*}, t^{n}\right) \hat{\xi}\left(\mathbf{x}, t^{n+1}\right) d \mathbf{x}\right|
$$$$
\leq \frac{1}{2} \int_{\Omega_{4}} \xi^{2}\left(\mathbf{x}, t^{n+1}\right) d \mathbf{x}+\frac{1}{2} \int_{\left(\Omega \backslash \Omega^{(O)}\left(t^{n}\right)\right) \cap\left(\left[x_{1}, b\right] \times\left[y_{1}, d\right]\right)} \xi^{2}\left(\mathbf{x}, t^{n}\right) d \mathbf{x}
$$

where

$$
\begin{aligned}
& K^{(3)}(\mathbf{x}):=\left(1-K^{(5)}(\mathbf{x})\right)^{2}, \quad K^{(5)}(\mathbf{x}):=\min \left\{1, \frac{\left|V_{1}\left(\mathbf{x}, t^{n+1}\right)\right| \Delta t}{\Delta x}\right\} \\
& K^{(4)}(\mathbf{x}):=\left(1-K^{(6)}(\mathbf{x})\right)^{2}, \quad K^{(6)}(\mathbf{x}):=\min \left\{1, \frac{\left|V_{2}\left(\mathbf{x}, t^{n+1}\right)\right| \Delta t}{\Delta y}\right\} .
\end{aligned}
$$




$$
\begin{aligned}
& x^{*}(x, y) \quad:=x-V_{1}\left(x, y, t^{n+1}\right) \Delta t, \quad y^{*}(x, y) \quad:=y-V_{2}\left(x, y, t^{n+1}\right) \Delta t, \\
& b^{*}\left(b, y_{1}\right):=b-V_{1}\left(b, y_{1}, t^{n+1}\right) \Delta t, \quad d^{*}\left(x_{1}, d\right):=d-V_{2}\left(x_{1}, d, t^{n+1}\right) \Delta t,
\end{aligned}
$$

$$
\begin{aligned}
& \Omega_{1}:=\left(\Omega \backslash \Omega^{(I)}\left(t^{n+1}\right)\right) \cap\left(\left[a, x_{1}\right] \times\left[y_{1}, d\right]\right), \\
& \Omega_{2}:=\left(\Omega \backslash \Omega^{(I)}\left(t^{n+1}\right)\right) \cap\left(\left[a, x_{1}\right] \times\left[c, y_{1}\right]\right), \\
& \Omega_{3}:=\left(\Omega \backslash \Omega^{(I)}\left(t^{n+1}\right)\right) \cap\left(\left[x_{1}, b\right] \times\left[c, y_{1}\right]\right), \\
& \Omega_{4}:=\left(\Omega \backslash \Omega^{(I)}\left(t^{n+1}\right)\right) \cap\left(\left[x_{1}, b\right] \times\left[y_{1}, d\right]\right) .
\end{aligned}
$$

Combining (4.3)-(4.4), we have bounded the first term on the right-hand side of (4.1). As with (4.3), we rewrite the second term on the right-hand side of (4.1) as

$$
\begin{aligned}
& \int_{\Omega} \eta\left(\mathbf{y}, t^{n}\right) \hat{\xi}\left(\mathbf{y}, t_{+}^{n}\right) d \mathbf{y} \\
& =\int_{\Omega \backslash \Omega^{(O)}\left(t^{n}\right)} \eta\left(\mathbf{y}, t^{n}\right) \hat{\xi}\left(\mathbf{y}, t_{+}^{n}\right) d \mathbf{y}+\int_{\Omega_{n}^{(O)}} \eta\left(\mathbf{y}, t^{n}\right) \hat{\xi}\left(\mathbf{y}, t_{+}^{n}\right) d \mathbf{y} \\
& (4.8)=\int_{\Omega \backslash \Omega^{(I)}\left(t^{n+1}\right)} \eta\left(\mathbf{x}^{*}, t^{n}\right) \hat{\xi}\left(\mathbf{x}, t^{n+1}\right)\left(\operatorname{det} \mathbf{J}\left(t^{n} ; \mathbf{x}, t^{n+1}\right) e^{-R\left(\mathbf{x}, t^{n+1}\right) \Delta t}-1\right) d \mathbf{x} \\
& +\int_{\Omega \backslash \Omega^{(I)}\left(t^{n+1}\right)} \eta\left(\mathbf{x}^{*}, t^{n}\right) \hat{\xi}\left(\mathbf{x}, t^{n+1}\right) d \mathbf{x} \\
& +\int_{S_{n}^{(O)}} \mathbf{v}(\mathbf{x}, t) \cdot \mathbf{n}(\mathbf{x}) \eta\left(\mathbf{x}^{*}(\mathbf{x}, t), t^{n}\right) \xi(\mathbf{x}, t) e^{-R(\mathbf{x}, t)\left(t-t^{n}\right)} d S .
\end{aligned}
$$

The first term on the right-hand side of $(4.8)$ is bounded by

$$
\begin{aligned}
& \left|\int_{\Omega \backslash \Omega^{(I)}\left(t^{n+1}\right)} \eta\left(\mathbf{x}^{*}, t^{n}\right) \hat{\xi}\left(\mathbf{x}, t^{n+1}\right)\left(\operatorname{det} \mathbf{J}\left(t^{n} ; \mathbf{x}, t^{n+1}\right) e^{-R\left(\mathbf{x}, t^{n+1}\right) \Delta t}-1\right) d \mathbf{x}\right| \\
& \leq M \Delta t \int_{\Omega \backslash \Omega^{(I)}\left(t^{n+1}\right)}\left|\eta\left(\mathbf{x}^{*}, t^{n}\right) \hat{\xi}\left(\mathbf{x}, t^{n+1}\right)\right| d \mathbf{x} \\
& \leq M \Delta t\left\|\eta\left(\mathbf{x}, t^{n}\right)\right\|_{L^{2}(\Omega)}\left\|\xi\left(\mathbf{x}, t^{n+1}\right)\right\|_{L^{2}(\Omega)} \\
& \leq M \Delta t\left\|\xi\left(\mathbf{x}, t^{n+1}\right)\right\|_{L^{2}(\Omega)}^{2}+M \Delta t h^{4}\left\|u\left(\mathbf{x}, t^{n}\right)\right\|_{H^{2}(\Omega)}^{2} .
\end{aligned}
$$

The second term on the right-hand side of (4.8) and the third term on the righthand side of (4.1) are combined in the error analysis. When $C r \geq 1$, where

$$
C r:=\max _{(\mathbf{x}, t) \in \bar{\Omega} \times[0, T]}\left\{\frac{\left|V_{1}(\mathbf{x}, t)\right| \Delta t}{\Delta x}, \frac{\left|V_{2}(\mathbf{x}, t)\right| \Delta t}{\Delta y}\right\},
$$

$h \leq M \Delta t$. These two terms are bounded by

$$
\begin{aligned}
& \left|\int_{\Omega \backslash \Omega^{(I)}\left(t^{n+1}\right)} \eta\left(\mathbf{x}^{*}, t^{n}\right) \hat{\xi}\left(\mathbf{x}, t^{n+1}\right) d \mathbf{x}-\int_{\Omega} \eta\left(\mathbf{x}, t^{n+1}\right) \hat{\xi}\left(\mathbf{x}, t^{n+1}\right) d \mathbf{x}\right| \\
& \leq\left|\int_{\Omega \backslash \Omega^{(I)}\left(t^{n+1}\right)} \eta\left(\mathbf{x}^{*}, t^{n}\right) \hat{\xi}\left(\mathbf{x}, t^{n+1}\right) d \mathbf{x}\right|+\left|\int_{\Omega} \eta\left(\mathbf{x}, t^{n+1}\right) \hat{\xi}\left(\mathbf{x}, t^{n+1}\right) d \mathbf{x}\right| \\
& \leq M\left\|\xi\left(\mathbf{x}, t^{n+1}\right)\right\|_{L^{2}(\Omega)}\left(\left\|\eta\left(\mathbf{x}, t^{n+1}\right)\right\|_{L^{2}(\Omega)}+\left\|\eta\left(\mathbf{x}, t^{n}\right)\right\|_{L^{2}(\Omega)}\right) \\
& \leq M h^{2}\left\|\xi\left(\mathbf{x}, t^{n+1}\right)\right\|_{L^{2}(\Omega)}\|u\|_{L^{\infty}\left(0, T ; H^{2}(\Omega)\right)} \\
& \leq M \Delta t\left\|\xi\left(\mathbf{x}, t^{n+1}\right)\right\|_{L^{2}(\Omega)}^{2}+M(\Delta t)^{3}\|u\|_{L^{\infty}\left(0, T ; H^{2}(\Omega)\right)}^{2} .
\end{aligned}
$$


When $C r<1$, we decompose these two terms as follows:

$$
\begin{aligned}
& \int_{\Omega \backslash \Omega^{(I)}\left(t^{n+1}\right)} \eta\left(\mathbf{x}^{*}, t^{n}\right) \hat{\xi}\left(\mathbf{x}, t^{n+1}\right) d \mathbf{x}-\int_{\Omega} \eta\left(\mathbf{x}, t^{n+1}\right) \hat{\xi}\left(\mathbf{x}, t^{n+1}\right) d \mathbf{x} \\
& =-\int_{\Omega \backslash \Omega^{(I)}\left(t^{n+1}\right)}\left[\int_{t^{n}}^{t^{n+1}} \eta_{t}(\mathbf{x}, t) d t\right] \hat{\xi}\left(\mathbf{x}, t^{n+1}\right) d \mathbf{x} \\
& \quad-\int_{\Omega^{(I)}\left(t^{n+1}\right)} \eta\left(\mathbf{x}, t^{n+1}\right) \hat{\xi}\left(\mathbf{x}, t^{n+1}\right) d \mathbf{x} \\
& \quad-\int_{\Omega \backslash \Omega^{(I)}\left(t^{n+1}\right)}\left[\eta\left(\mathbf{x}, t^{n}\right)-\eta\left(\mathbf{x}^{*}, t^{n}\right)\right] \hat{\xi}\left(\mathbf{x}, t^{n+1}\right) d \mathbf{x} .
\end{aligned}
$$

The first term on the right-hand side of $(4.10)$ is bounded by

$$
\begin{aligned}
& \left|\int_{\Omega \backslash \Omega^{(I)}\left(t^{n+1}\right)}\left[\int_{t^{n}}^{t^{n+1}} \eta_{t}(\mathbf{x}, t) d t\right] \hat{\xi}\left(\mathbf{x}, t^{n+1}\right) d \mathbf{x}\right| \\
& \leq(\Delta t)^{1 / 2} \int_{\Omega \backslash \Omega^{(I)}\left(t^{n+1}\right)}\left[\int_{t^{n}}^{t^{n+1}} \eta_{t}^{2}(\mathbf{x}, t) d t\right]^{1 / 2}\left|\hat{\xi}\left(\mathbf{x}, t^{n+1}\right)\right| d \mathbf{x} \\
& \leq M \Delta t\left\|\xi\left(\mathbf{x}, t^{n+1}\right)\right\|_{L^{2}(\Omega)}^{2}+M\left\|\eta_{t}\right\|_{L^{2}\left(t^{n}, t^{n+1} ; L^{2}(\Omega)\right)}^{2} \\
& \leq M \Delta t\left\|\xi\left(\mathbf{x}, t^{n+1}\right)\right\|_{L^{2}(\Omega)}^{2}+M h^{4}\left\|u_{t}\right\|_{L^{2}\left(t^{n}, t^{n+1} ; H^{2}(\Omega)\right)} .
\end{aligned}
$$

However, the remaining two terms on the right-hand side of (4.10) are more difficult to bound. The techniques in the previous analyses for MMOC $[10,12]$ lead only to a suboptimal-order error estimate that does not reflect the strength of the ELLAM scheme. To derive an optimal-order error estimate, we develop new techniques to analyze these terms and present the detailed analyses in Lemmas 2 and 3 in sections 6 and 7 ; there we obtain

$$
\begin{aligned}
& \left|\int_{\Omega^{(I)}\left(t^{n+1}\right)} \eta\left(\mathbf{x}, t^{n+1}\right) \hat{\xi}\left(\mathbf{x}, t^{n+1}\right) d \mathbf{x}+\int_{\Omega \backslash \Omega^{(I)}\left(t^{n+1}\right)}\left[\eta\left(\mathbf{x}, t^{n}\right)-\eta\left(\mathbf{x}^{*}, t^{n}\right)\right] \hat{\xi}\left(\mathbf{x}, t^{n+1}\right) d \mathbf{x}\right| \\
& \leq \varepsilon_{1} \int_{S_{n}^{(O)}} \mathbf{v} \cdot \mathbf{n} \xi^{2}(\mathbf{x}, t) d S+\varepsilon_{1} \Delta x \Delta y\left[K^{(5)}\left(\mathbf{x}_{1,1}\right)+K^{(6)}\left(\mathbf{x}_{1,1}\right)\right] \xi^{2}\left(\mathbf{x}_{1,1}, t^{n+1}\right) \\
& +\varepsilon_{1} \Delta x \int_{y_{1}}^{d} K^{(5)}\left(x_{1}, y\right) \xi^{2}\left(x_{1}, y, t^{n+1}\right) d y+\varepsilon_{1} \Delta y \int_{x_{1}}^{b} K^{(6)}\left(x, y_{1}\right) \xi^{2}\left(x, y_{1}, t^{n+1}\right) d x \\
& +M \Delta t\left\|\xi\left(\mathbf{x}, t^{n+1}\right)\right\|_{L^{2}(\Omega)}^{2}+M \Delta t\left[h^{4}+(\Delta t)^{2}\right]\left\|u\left(\mathbf{x}, t^{n+1}\right)\right\|_{H^{3}(\Omega)}^{2},
\end{aligned}
$$

where $\varepsilon_{1}=0$ for $C r \geq 1$ (recall (4.9)).

The third term on the right-hand side of $(4.8)$ is bounded by

$$
\begin{aligned}
& \left|\int_{S_{n}^{(O)}} \mathbf{v} \cdot \mathbf{n} \eta\left(\mathbf{x}^{*}(\mathbf{x}, t), t^{n}\right) \xi(\mathbf{x}, t) e^{-R(\mathbf{x}, t)\left(t-t^{n}\right)} d S\right| \\
& \leq M\left[\int_{S_{n}^{(O)}} \mathbf{v} \cdot \mathbf{n} \xi^{2}(\mathbf{x}, t) d S\right]^{1 / 2}\left[\int_{S_{n}^{(O)}} \mathbf{v} \cdot \mathbf{n} \eta^{2}\left(\mathbf{x}^{*}(\mathbf{x}, t), t^{n}\right) d S\right]^{1 / 2} \\
& \leq \varepsilon_{2} \int_{S_{n}^{(O)}} \mathbf{v} \cdot \mathbf{n} \xi^{2}(\mathbf{x}, t) d S+M \int_{\Omega^{(O)}\left(t^{n}\right)} \eta^{2}\left(\mathbf{x}, t^{n}\right) d \mathbf{x} \\
& \leq \varepsilon_{2} \int_{S_{n}^{(O)}} \mathbf{v} \cdot \mathbf{n} \xi^{2}(\mathbf{x}, t) d S+M(\Delta t)^{3}\left\|u\left(\mathbf{x}, t^{n}\right)\right\|_{H^{2}(\Omega)}^{2} \\
& +\lambda M \Delta t\left[h^{4}+(\Delta t)^{2}\right]\left\|u\left(\mathbf{x}, t^{n}\right)\right\|_{H^{3}(\Omega)}^{2},
\end{aligned}
$$


where $\lambda=1$ if $C r<1$ and 0 otherwise. At the last step we have used (6.2), which will appear in section 6 .

Recalling (3.10), $\Pi^{\mathrm{I}} u(\mathbf{x}, t)=u\left(\mathbf{x}, t_{n, I C-1}\right)$ for $\mathbf{x} \in \Gamma^{(O)}$ and $t \in\left[t^{n}, t_{n, I C-1}\right]$. When $C r \geq 1, h \leq M \Delta t$. Hence,

$$
\begin{aligned}
\int_{S_{n}^{(O)}} \eta^{2}(\mathbf{x}, t) d S & \leq M\left[h^{2}+\left(\Delta t_{f}\right)^{2}\right]\left(\left\|u_{s}\right\|_{L^{2}\left(S_{n}^{(O)}\right)}^{2}+\left\|u_{t}\right\|_{L^{2}\left(S_{n}^{(O)}\right)}^{2}\right) \\
& \leq M(\Delta t)^{2}\left(\left\|u_{s}\right\|_{L^{2}\left(S_{n}^{(O)}\right)}^{2}+\left\|u_{t}\right\|_{L^{2}\left(S_{n}^{(O)}\right)}^{2}\right)
\end{aligned}
$$

where $u_{s}$ is the tangential derivative of $u$ on $\Gamma$ and $\Delta t_{f}$ is defined in (2.19).

When $C r<1, t_{n, I C-1}=t^{n+1}$. So, $\Pi^{\mathrm{I}} u(\mathbf{x}, t)=u\left(\mathbf{x}, t^{n+1}\right)$ for $(\mathbf{x}, t) \in S_{n}^{(O)}$. Thus,

$$
\begin{aligned}
\int_{S_{n}^{(O)}} \eta^{2}(\mathbf{x}, t) d S & \leq M \Delta t \int_{\Gamma^{(O)}} \eta^{2}\left(\mathbf{x}, t^{n+1}\right) d s+M \int_{S_{n}^{(O)}}\left[\int_{t}^{t^{n+1}} u_{t}(\mathbf{x}, \theta) d \theta\right]^{2} d S \\
& \leq \Delta t h^{4}\left\|u_{s s}\right\|_{L^{\infty}\left(0, T ; L^{2}\left(S_{n}^{(O)}\right)\right)}^{2}+M(\Delta t)^{2}\left\|u_{t}\right\|_{L^{2}\left(S_{n}^{(O)}\right)}^{2} .
\end{aligned}
$$

Combining the two preceding estimates, we bound the fourth term on the righthand side of (4.1):

$$
\begin{aligned}
& \left|\int_{S_{n}^{(O)}} \mathbf{v}(\mathbf{x}, t) \cdot \mathbf{n}(\mathbf{x}) \eta(\mathbf{x}, t) \xi(\mathbf{x}, t) d S\right| \\
& \quad \leq\left[\int_{S_{\eta}^{(O)}} \mathbf{v}(\mathbf{x}, t) \cdot \mathbf{n}(\mathbf{x}) \eta^{2}(\mathbf{x}, t) d S\right]^{1 / 2}\left[\int_{S_{\eta}^{(O)}} \mathbf{v}(\mathbf{x}, t) \cdot \mathbf{n}(\mathbf{x}) \xi^{2}(\mathbf{x}, t) d S\right]^{1 / 2} \\
& \quad \leq \varepsilon_{2} \int_{S_{n}^{(O)}} \mathbf{v}(\mathbf{x}, t) \cdot \mathbf{n}(\mathbf{x}) \xi^{2}(\mathbf{x}, t) d S+M \int_{S_{n}^{(O)}} \eta^{2}(\mathbf{x}, t) d S \\
& \quad \leq \varepsilon_{2} \int_{S_{n}^{(O)}} \mathbf{v}(\mathbf{x}, t) \cdot \mathbf{n}(\mathbf{x}) \xi^{2}(\mathbf{x}, t) d S+\lambda M \Delta t h^{4}\|u\|_{L^{\infty}\left(0, T ; H^{3}(\Omega)\right)}^{2} \\
& \quad+M(\Delta t)^{2}\left[\|u\|_{L^{2}\left(t^{n}, t^{n+1} ; H^{2}(\Omega)\right)}^{2}+\left\|u_{t}\right\|_{L^{2}\left(t^{n}, t^{n+1} ; H^{1}(\Omega)\right)}^{2}\right]
\end{aligned}
$$

where $\lambda=1$ if $C r<1$ and 0 otherwise. At the last step we have used the trace theorem in the Sobolev spaces

$$
\|u(\cdot, t)\|_{L^{2}(\Gamma)} \leq M\|u(\cdot, t)\|_{H^{1}(\Omega)}
$$

The last term on the right-hand side of (4.1) is estimated in Lemma 4 in section 8. We obtain

$$
\begin{aligned}
& |E(\hat{\xi})| \leq \varepsilon_{3} \int_{S_{n}^{(O)}} \mathbf{v} \cdot \mathbf{n} \xi^{2}(\mathbf{x}, t) d S+\varepsilon_{3} \int_{\Omega^{(I)}\left(t^{n+1}\right) \cap\left(\left[x_{1}, b\right] \times\left[y_{1}, d\right]\right)} \xi^{2}\left(\mathbf{x}, t^{n+1}\right) d \mathbf{x} \\
& \quad+\varepsilon_{3} \Delta x \int_{y_{1}}^{d} K^{(5)}\left(x_{1}, y\right) \xi^{2}\left(x_{1}, y, t^{n+1}\right) d y \\
& \quad+\varepsilon_{3} \Delta y \int_{x_{1}}^{b} K^{(6)}\left(x, y_{1}\right) \xi^{2}\left(x, y_{1}, t^{n+1}\right) d x \\
& \quad+\varepsilon_{3} \Delta x \Delta y K^{(5)}\left(\mathbf{x}_{1,1}\right) K^{(6)}\left(\mathbf{x}_{1,1}\right) \xi^{2}\left(\mathbf{x}_{1,1}, t^{n+1}\right)+M \Delta t\left\|\xi\left(\mathbf{x}, t^{n+1}\right)\right\|_{L^{2}(\Omega)}^{2} \\
& \quad+M \Delta t\|\xi(\mathbf{x}, t)\|_{L^{2}\left(S_{n}^{(O)}\right)}^{2}+M(\Delta t)^{3}\|u\|_{L^{\infty}\left(0, T ; H^{1}(\Omega)\right)}^{2} \\
& \quad+M(\Delta t)^{2}\left[\|f\|_{L^{2}\left(t^{n}, t^{n+1} ; L^{2}(\Omega)\right)}^{2}+\left\|f_{\tau}\right\|_{L^{2}\left(t^{n}, t^{n+1} ; L^{2}(\Omega)\right)}^{2}\right]
\end{aligned}
$$

where $f_{\tau}$ is the derivative of $f$ along the (approximate) characteristics. 
Incorporating (4.2)-(4.4), (4.8)-(4.12), and (4.14) into (4.1), we obtain

$$
\begin{aligned}
& \int_{y_{1}}^{d} \int_{x_{1}}^{b} \xi^{2}\left(\mathbf{x}, t^{n+1}\right) d \mathbf{x}+\frac{\Delta x}{2} \int_{y_{1}}^{d} \xi^{2}\left(x_{1}, y, t^{n+1}\right) d y \\
& +\frac{\Delta y}{2} \int_{x_{1}}^{b} \xi^{2}\left(x, y_{1}, t^{n+1}\right) d x+\frac{\Delta x \Delta y}{4} \xi^{2}\left(\mathbf{x}_{1,1}, t^{n+1}\right)+\int_{S_{n}^{(O)}} \mathbf{v} \cdot \mathbf{n} \xi^{2}(\mathbf{x}, t) d S \\
& \leq \frac{1}{2} \int_{y_{1}}^{d} \int_{x_{1}}^{b} \xi^{2}\left(\mathbf{x}, t^{n+1}\right) d \mathbf{x}+\frac{1}{2} \int_{y_{1}}^{d} \int_{x_{1}}^{b} \xi^{2}\left(\mathbf{x}, t^{n}\right) d \mathbf{x} \\
& +\frac{\Delta x \Delta y}{8}\left[K^{(3)}\left(\mathbf{x}_{1,1}\right) K^{(4)}\left(\mathbf{x}_{1,1}\right)+8 \varepsilon_{1} K^{(5)}\left(\mathbf{x}_{1,1}\right)+8 \varepsilon_{1} K^{(6)}\left(\mathbf{x}_{1,1}\right)\right. \\
& \left.+8 \varepsilon_{3} K^{(5)}\left(\mathbf{x}_{1,1}\right) K^{(6)}\left(\mathbf{x}_{1,1}\right)\right] \xi^{2}\left(\mathbf{x}_{1,1}, t^{n+1}\right) \\
& \times \frac{\Delta x \Delta y}{8} \xi^{2}\left(\mathbf{x}_{1,1}, t^{n}\right)+\frac{\Delta x}{4} \int_{y_{1}}^{d} \xi^{2}\left(x_{1}, y, t^{n}\right) d y+\frac{\Delta y}{4} \int_{x_{1}}^{b} \xi^{2}\left(x, y_{1}, t^{n}\right) d x \\
& +\frac{\Delta x}{4} \int_{y_{1}}^{d}\left[K^{(3)}\left(x_{1}, y\right)+4\left(\varepsilon_{1}+\varepsilon_{3}\right) K^{(5)}\left(x_{1}, y\right)\right] \xi^{2}\left(x_{1}, y, t^{n+1}\right) d y \\
& +\frac{\Delta y}{4} \int_{x_{1}}^{b}\left[K^{(4)}\left(x, y_{1}\right)+4\left(\varepsilon_{1}+\varepsilon_{3}\right) K^{(6)}\left(x, y_{1}\right)\right] \xi^{2}\left(x, y_{1}, t^{n+1}\right) d x \\
& +\left(\frac{1}{2}+M \Delta t+\varepsilon_{1}+2 \varepsilon_{2}+\varepsilon_{3}\right) \int_{S_{n}^{(O)}} \mathbf{v}(\mathbf{x}, t) \cdot \mathbf{n}(\mathbf{x}) \xi^{2}(\mathbf{x}, t) d S \\
& +M \Delta t\|\xi(\mathbf{x}, t)\|_{L^{2}\left(S_{n}^{(O)}\right)}^{2}+M \Delta t\left\|\xi\left(\mathbf{x}, t^{n+1}\right)\right\|_{L^{2}(\Omega)}^{2}+M \Delta t\left\|\xi\left(\mathbf{x}, t^{n}\right)\right\|_{L^{2}(\Omega)}^{2} \\
& +M \Delta t\left[h^{4}+(\Delta t)^{2}\right]\|u\|_{L^{\infty}\left(0, T ; H^{2}(\Omega)\right)}^{2}+M(\Delta t)^{2}\left\|u_{t}\right\|_{L^{2}\left(t^{n}, t^{n+1} ; H^{1}(\Omega)\right)}^{2} \\
& +M h^{4}\left\|u_{t}\right\|_{L^{2}\left(t^{n}, t^{n+1} ; H^{2}(\Omega)\right)}^{2}+\lambda M \Delta t\left[h^{4}+(\Delta t)^{2}\right]\|u\|_{L^{\infty}\left(0, T ; H^{3}(\Omega)\right)}^{2} \\
& +M(\Delta t)^{2}\left[\|f\|_{L^{2}\left(t^{n}, t^{n+1} ; L^{2}(\Omega)\right)}^{2}+\left\|f_{\tau}\right\|_{L^{2}\left(t^{n}, t^{n+1} ; L^{2}(\Omega)\right)}^{2}\right],
\end{aligned}
$$

where we have used the fact that

$$
\begin{aligned}
& \int_{\Omega_{n}^{(O)}} \xi^{2}\left(\mathbf{x}, t^{n}\right) d \mathbf{x}+\int_{\left(\Omega \backslash \Omega^{(O)}\left(t^{n}\right)\right) \cap\left(\left[x_{1}, b\right] \times\left[y_{1}, d\right]\right)} \xi^{2}\left(\mathbf{x}, t^{n}\right) d \mathbf{x} \\
& \quad \leq \int_{y_{1}}^{d} \int_{x_{1}}^{b} \xi^{2}\left(\mathbf{x}, t^{n}\right) d \mathbf{x}+\int_{d^{*}\left(x_{1}, d\right)}^{x_{a}^{x_{1}}} \xi^{2}\left(\mathbf{x}, t^{n}\right) d \mathbf{x}+\int_{b^{*}\left(b, y_{1}\right)}^{b} \int_{c}^{y_{1}} \xi^{2}\left(\mathbf{x}, t^{n}\right) d \mathbf{x} \\
& \quad \leq \int_{y_{1}}^{d} \int_{x_{1}}^{b} \xi^{2}\left(\mathbf{x}, t^{n}\right) d \mathbf{x}+\frac{\Delta x}{4} \int_{d^{*}\left(x_{1}, d\right)}^{d} \xi^{2}\left(x_{1}, y, t^{n}\right) d y+\frac{\Delta y}{4} \int_{b^{*}\left(b, y_{1}\right)}^{b} \xi^{2}\left(x, y_{1}, t^{n}\right) d x .
\end{aligned}
$$

In (4.15) we choose $\varepsilon_{1}=\varepsilon_{2}=\varepsilon_{3}=1 / 24$. By the definitions of $K^{(3)}(\mathbf{x})-K^{(6)}(\mathbf{x})$ in (4.5), we have the following estimates:

$$
\begin{aligned}
& K^{(3)}(\mathbf{x})+4\left(\varepsilon_{1}+\varepsilon_{3}\right) K^{(5)}(\mathbf{x}) \\
& \quad \leq\left[1-K^{(5)}(\mathbf{x})\right]^{2}+K^{(5)}(\mathbf{x})=1-K^{(5)}(\mathbf{x})\left[1-K^{(5)}(\mathbf{x})\right] \leq 1, \\
& K^{(4)}(\mathbf{x})+4\left(\varepsilon_{1}+\varepsilon_{3}\right) K^{(6)}(\mathbf{x}) \\
& \quad \leq\left[1-K^{(6)}(\mathbf{x})\right]^{2}+K^{(6)}(\mathbf{x})=1-K^{(6)}(\mathbf{x})\left[1-K^{(6)}(\mathbf{x})\right] \leq 1, \\
& K^{(3)}(\mathbf{x}) K^{(4)}(\mathbf{x})+8 \varepsilon_{1} K^{(5)}(\mathbf{x})+8 \varepsilon_{1} K^{(6)}(\mathbf{x})+8 \varepsilon_{3} K^{(5)}(\mathbf{x}) K^{(6)}(\mathbf{x}) \\
& \quad \leq\left[1-K^{(5)}(\mathbf{x})\right]\left[1-K^{(6)}(\mathbf{x})\right]+\frac{1}{3}\left[K^{(5)}(\mathbf{x})+K^{(6)}(\mathbf{x})+K^{(5)}(\mathbf{x}) K^{(6)}(\mathbf{x})\right] \\
& \quad=1-\frac{2}{3} K^{(5)}(\mathbf{x})\left[1-K^{(6)}(\mathbf{x})\right]-\frac{2}{3} K^{(6)}(\mathbf{x})\left[1-K^{(5)}(\mathbf{x})\right] \leq 1 .
\end{aligned}
$$


Canceling the corresponding terms in (4.15) and then multiplying both sides by 2 , we obtain

$$
\begin{aligned}
\int_{y_{1}}^{d} & \int_{x_{1}}^{b} \xi^{2}\left(\mathbf{x}, t^{n+1}\right) d \mathbf{x}+\frac{\Delta x}{2} \int_{y_{1}}^{d} \xi^{2}\left(x_{1}, y, t^{n+1}\right) d y+\frac{\Delta y}{2} \int_{x_{1}}^{b} \xi^{2}\left(x, y_{1}, t^{n+1}\right) d x \\
& +\frac{\Delta x \Delta y}{4} \xi^{2}\left(\mathbf{x}_{1,1}, t^{n+1}\right)+\frac{2}{3} \int_{S_{n}^{(O)}} \mathbf{v}(\mathbf{x}, t) \cdot \mathbf{n}(\mathbf{x}) \xi^{2}(\mathbf{x}, t) d S \\
\leq & \frac{1}{2} \int_{y_{1}}^{d} \int_{x_{1}}^{b} \xi^{2}\left(\mathbf{x}, t^{n}\right) d \mathbf{x}+\frac{\Delta x \Delta y}{4} \xi^{2}\left(\mathbf{x}_{1,1}, t^{n}\right)+\frac{\Delta x}{2} \int_{y_{1}}^{d} \xi^{2}\left(x_{1}, y, t^{n}\right) d y \\
& +\frac{\Delta y}{2} \int_{x_{1}}^{b} \xi^{2}\left(x, y_{1}, t^{n}\right) d x+M \Delta t \int_{S_{n}^{(O)}} \mathbf{v}(\mathbf{x}, t) \cdot \mathbf{n}(\mathbf{x}) \xi^{2}(\mathbf{x}, t) d S \\
& +M \Delta t\|\xi(\mathbf{x}, t)\|_{L^{2}\left(S_{n}^{(O)}\right)}^{2}+M \Delta t\left\|\xi\left(\mathbf{x}, t^{n+1}\right)\right\|_{L^{2}(\Omega)}^{2}+M \Delta t\left\|\xi\left(\mathbf{x}, t^{n}\right)\right\|_{L^{2}(\Omega)}^{2} \\
& +M \Delta t\left[h^{4}+(\Delta t)^{2}\right]\|u\|_{L^{\infty}\left(0, T ; H^{2}(\Omega)\right)}^{2}+M(\Delta t)^{2}\left\|u_{t}\right\|_{L^{2}\left(t^{n}, t^{n+1} ; H^{1}(\Omega)\right)}^{2} \\
& +M h^{4}\left\|u_{t}\right\|_{L^{2}\left(t^{n}, t^{n+1} ; H^{2}(\Omega)\right)}^{2}+\lambda M \Delta t\left[h^{4}+(\Delta t)^{2}\right]\|u\|_{L^{\infty}\left(0, T ; H^{3}(\Omega)\right)}^{2} \\
& +M(\Delta t)^{2}\left[\|f\|_{L^{2}\left(t^{n}, t^{n+1} ; L^{2}(\Omega)\right)}^{2}+\left\|f_{\tau}\right\|_{L^{2}\left(t^{n}, t^{n+1} ; L^{2}(\Omega)\right)}^{2}\right] .
\end{aligned}
$$

Summing the preceding equation over $n$, we obtain

$$
\begin{aligned}
& \left\|\xi\left(\mathbf{x}, t^{n+1}\right)\right\|_{L^{2}(\Omega)}^{2}+\frac{2}{3} \int_{0}^{t^{n+1}} \int_{\Gamma^{(O)}} \mathbf{v}(\mathbf{x}, t) \cdot \mathbf{n}(\mathbf{x}) \xi^{2}(\mathbf{x}, t) d S \\
& \leq \int_{y_{1}}^{d} \int_{x_{1}}^{b} \xi^{2}\left(\mathbf{x}, t^{n+1}\right) d \mathbf{x}+\frac{\Delta x}{2} \int_{y_{1}}^{d} \xi^{2}\left(x_{1}, y, t^{n+1}\right) d y+\frac{\Delta y}{2} \int_{x_{1}}^{b} \xi^{2}\left(x, y_{1}, t^{n+1}\right) d x \\
& \quad+\frac{\Delta x \Delta y}{4} \xi^{2}\left(\mathbf{x}_{1,1}, t^{n+1}\right)+\frac{2}{3} \int_{0}^{t^{n+1}} \int_{\Gamma^{(O)}} \mathbf{v}(\mathbf{x}, t) \cdot \mathbf{n}(\mathbf{x}) \xi^{2}(\mathbf{x}, t) d S \\
& \leq \\
& \quad M \Delta t \sum_{k=0}^{n+1}\left\|\xi\left(\mathbf{x}, t^{k}\right)\right\|_{L^{2}(\Omega)}^{2}+M \Delta t \int_{S_{n}^{(O)}} \mathbf{v}(\mathbf{x}, t) \cdot \mathbf{n}(\mathbf{x}) \xi^{2}(\mathbf{x}, t) d S \\
& \quad+M\left[h^{4}+(\Delta t)^{2}\right]\|u\|_{L^{\infty}\left(0, T ; H^{2}(\Omega)\right)}^{2}+M(\Delta t)^{2}\left\|u_{t}\right\|_{L^{2}\left(0, T ; H^{1}(\Omega)\right)}^{2} \\
& \quad+M h^{4}\left\|u_{t}\right\|_{L^{2}\left(0, T ; H^{2}(\Omega)\right)}^{2}+\lambda M\left[h^{4}+(\Delta t)^{2}\right]\|u\|_{L^{\infty}\left(0, T ; H^{3}(\Omega)\right)}^{2} \\
& \quad+M(\Delta t)^{2}\left[\|f\|_{L^{2}\left(0, T ; L^{2}(\Omega)\right)}^{2}+\left\|f_{\tau}\right\|_{L^{2}\left(0, T ; L^{2}(\Omega)\right)}^{2}\right]
\end{aligned}
$$

Taking $\Delta t$ sufficiently small such that $M \Delta t \leq 1 / 2$ and applying Gronwall's inequality to the previous equation, we obtain the following estimate:

$$
\begin{aligned}
\|\xi\|_{\hat{L}^{\infty}\left(0, T ; L^{2}(\Omega)\right)}+\|\xi\|_{L^{2}\left(0, T ; L^{2}\left(\Gamma^{(O)}\right)\right)} \\
\leq M\left[h^{2}+\Delta t\right]\left[\|u\|_{L^{\infty}\left(0, T ; H^{2}(\Omega)\right)}+\lambda\|u\|_{L^{\infty}\left(0, T ; H^{3}(\Omega)\right)}\right] \\
\quad+M \Delta t\left\|u_{t}\right\|_{L^{2}\left(0, T ; H^{1}(\Omega)\right)}+M h^{2}\left\|u_{t}\right\|_{L^{2}\left(0, T ; H^{2}(\Omega)\right)} \\
\quad+M \Delta t\left[\|f\|_{L^{2}\left(0, T ; L^{2}(\Omega)\right)}+\left\|f_{\tau}\right\|_{L^{2}\left(0, T ; L^{2}(\Omega)\right)}\right]
\end{aligned}
$$


where the discrete norm $\|\cdot\|_{\hat{L}^{\infty}\left(0, T ; L^{2}(\Omega)\right)}$ is defined in (3.1). $\lambda=1$ if $C r<1$ and 0 otherwise.

Combining (4.16) with the estimate (3.2), we have proven the main theorem.

THEOREM 1. Let $u(\mathbf{x}, t)$ be the exact solution of (2.1) satisfying $u \in L^{\infty}(0, T$; $\left.H^{3}(\Omega)\right)$ and $u_{t} \in L^{2}\left(0, T ; H^{2}(\Omega)\right)$, and let $U\left(\mathbf{x}, t^{n}\right)$ be the numerical solution given by the ELLAM scheme (2.21). Then the following optimal-order $L^{2}$ error estimate holds:

$$
\begin{aligned}
\| U- & u\left\|_{\hat{L}^{\infty}\left(0, T ; L^{2}(\Omega)\right)}+\right\| U-u \|_{L^{2}\left(0, T ; L^{2}\left(\Gamma^{(O)}\right)\right)} \\
\leq & M\left[h^{2}+\Delta t\right]\left[\|u\|_{L^{\infty}\left(0, T ; H^{2}(\Omega)\right)}+\|u\|_{L^{\infty}\left(0, T ; H^{3}(\Omega)\right)}\right] \\
& +M \Delta t\left\|u_{t}\right\|_{L^{2}\left(0, T ; H^{1}(\Omega)\right)}+M h^{2}\left\|u_{t}\right\|_{L^{2}\left(0, T ; H^{2}(\Omega)\right)} \\
& +M \Delta t\left[\|f\|_{L^{2}\left(0, T ; L^{2}(\Omega)\right)}+\left\|f_{\tau}\right\|_{L^{2}\left(0, T ; L^{2}(\Omega)\right)}\right]
\end{aligned}
$$

where $\lambda=1$ if $C r<1$ and 0 otherwise.

REMARK 1. For simplicity of presentation, we have derived an optimal-order $L^{2}$ error estimate (4.17) for the ELLAM scheme (2.21) in two space dimensions. Notice that a similar error expansion to (3.6) for the blending or Boolean interpolation holds for higher space dimensions. The error estimate (4.17) holds for the ELLAM scheme defined in higher space dimensions.

5. Proof of Lemma 1. The use of the nonconventional test functions $\hat{w}$ in the ELLAM scheme (2.21) requires a very tight estimate (5.1) to bound the second term on the right-hand side of (4.3), which is proved in Lemma 1 below.

LEMMA 1. Let $\xi$ and $\hat{\xi}$ be defined in (3.11)-(3.12). Then the following estimate holds for the second term on the right-hand side of (4.3):

$$
\begin{aligned}
& \left|\int_{\Omega \backslash \Omega^{(I)}\left(t^{n+1}\right)} \xi\left(\mathbf{x}^{*}, t^{n}\right) \hat{\xi}\left(\mathbf{x}, t^{n+1}\right) d \mathbf{x}\right| \\
& \leq \frac{1}{2} \int_{\Omega_{4}} \xi^{2}\left(\mathbf{x}, t^{n+1}\right) d \mathbf{x}+\frac{1}{2} \int_{\left(\Omega \backslash \Omega^{(O)}\left(t^{n}\right)\right) \cap\left(\left[x_{1}, b\right] \times\left[y_{1}, d\right]\right)} \xi^{2}\left(\mathbf{x}, t^{n}\right) d \mathbf{x} \\
& +\frac{\Delta x}{4} \int_{y_{1}}^{d} K^{(3)}\left(x_{1}, y\right) \xi^{2}\left(x_{1}, y, t^{n+1}\right) d y+\frac{\Delta y}{4} \int_{x_{1}}^{b} K^{(4)}\left(x, y_{1}\right) \xi^{2}\left(x, y_{1}, t^{n+1}\right) d x \\
& +\frac{\Delta x}{4} \int_{y_{1}}^{d^{*}\left(x_{1}, d\right)} \xi^{2}\left(x_{1}, y, t^{n}\right) d y+\frac{\Delta y}{4} \int_{x_{1}}^{b^{*}\left(b, y_{1}\right)} \xi^{2}\left(x, y_{1}, t^{n}\right) d x \\
& +\frac{\Delta x \Delta y}{8} K^{(3)}\left(\mathbf{x}_{1,1}\right) K^{(4)}\left(\mathbf{x}_{1,1}\right) \xi^{2}\left(\mathbf{x}_{1,1}, t^{n+1}\right)+\frac{\Delta x \Delta y}{8} \xi^{2}\left(\mathbf{x}_{1,1}, t^{n}\right) \\
& +M \Delta t\left\|\xi\left(\mathbf{x}, t^{n+1}\right)\right\|_{L^{2}(\Omega)}^{2}+M \Delta t\left\|\xi\left(\mathbf{x}, t^{n}\right)\right\|_{L^{2}(\Omega)}^{2},
\end{aligned}
$$

where $K^{(3)}(\mathbf{x})$ and $K^{(4)}(\mathbf{x})$ are defined in $(4.5)$.

Proof. We prove this lemma by considering two different cases.

Case 1 . $C r<1$. In this case, the curves $\tilde{a}(y)$ and $\tilde{c}(x)$ defined by

$$
\begin{aligned}
& a=r_{1}\left(t^{n} ; \tilde{a}(y), y, t^{n+1}\right)=\tilde{a}(y)-V_{1}\left(\tilde{a}(y), y, t^{n+1}\right) \Delta t, \\
& c=r_{2}\left(t^{n} ; x, \tilde{c}(x), t^{n+1}\right)=\tilde{c}(x)-V_{2}\left(x, \tilde{c}(x), t^{n+1}\right) \Delta t
\end{aligned}
$$

fall in the regions $\left[a, x_{1}\right] \times[c, d]$ and $[a, b] \times\left[c, y_{1}\right]$, respectively, where

$$
\mathbf{r}\left(\theta ; \mathbf{x}, t^{n+1}\right)=\left(r_{1}\left(\theta ; x, y, t^{n+1}\right), r_{2}\left(\theta ; x, y, t^{n+1}\right)\right) .
$$


Hence, the domain $\Omega \backslash \Omega^{(I)}\left(t^{n+1}\right)$ is decomposed as

$$
\Omega \backslash \Omega^{(I)}\left(t^{n+1}\right)=\Omega_{1} \cup \Omega_{2} \cup \Omega_{3} \cup \Omega_{4}
$$

with $\Omega_{1}$ through $\Omega_{4}$ being defined in (4.7).

Moreover, the $K^{(3)}(\mathbf{x})$ and $K^{(4)}(\mathbf{x})$ defined in (4.5) are now reduced to

$$
K^{(3)}(\mathbf{x})=\left(1-\frac{V_{1}\left(\mathbf{x}, t^{n+1}\right) \Delta t}{\Delta x}\right)^{2}, \quad K^{(4)}(\mathbf{x})=\left(1-\frac{V_{2}\left(\mathbf{x}, t^{n+1}\right) \Delta t}{\Delta y}\right)^{2} .
$$

In this section we also frequently use the following relations:

$$
\begin{aligned}
V_{1}\left(x, y, t^{n+1}\right) \Delta t-V_{1}\left(x_{1}, y, t^{n+1}\right) \Delta t & =\mathcal{O}(h \Delta t), & x \in\left[a, x_{1}\right], & y \in[c, d], \\
V_{1}\left(x, y, t^{n+1}\right) \Delta t-V_{1}\left(x_{1}, y_{1}, t^{n+1}\right) \Delta t & =\mathcal{O}(h \Delta t), & x \in\left[a, x_{1}\right], & y \in\left[c, y_{1}\right], \\
\tilde{a}(y)-a-V_{1}\left(x, y, t^{n+1}\right) \Delta t & =\mathcal{O}(h \Delta t) & &
\end{aligned}
$$

or, equivalently,

$$
\begin{aligned}
K^{(3)}(x, y)-K^{(3)}\left(x_{1}, y\right) & =\mathcal{O}(\Delta t), \quad x \in\left[a, x_{1}\right], \quad y \in[c, d], \\
K^{(3)}(x, y)-K^{(3)}\left(x_{1}, y_{1}\right) & =\mathcal{O}(\Delta t), \quad x \in\left[a, x_{1}\right], \quad y \in\left[c, y_{1}\right] .
\end{aligned}
$$

We split the left-hand side of (5.1) based on the decomposition (5.3):

$$
\begin{aligned}
& \int_{\Omega \backslash \Omega^{(I)}\left(t^{n+1}\right)} \xi\left(\mathbf{x}^{*}, t^{n}\right) \hat{\xi}\left(\mathbf{x}, t^{n+1}\right) d \mathbf{x} \\
& =\int_{\Omega_{1}} \xi\left(\mathbf{x}^{*}, t^{n}\right) \xi\left(x_{1}, y, t^{n+1}\right) d \mathbf{x}+\int_{\Omega_{2}} \xi\left(\mathbf{x}^{*}, t^{n}\right) \xi\left(\mathbf{x}_{1,1}, t^{n+1}\right) d \mathbf{x} \\
& \quad+\int_{\Omega_{3}} \xi\left(\mathbf{x}^{*}, t^{n}\right) \xi\left(x, y_{1}, t^{n+1}\right) d \mathbf{x}+\int_{\Omega_{4}} \xi\left(\mathbf{x}^{*}, t^{n}\right) \xi\left(\mathbf{x}, t^{n+1}\right) d \mathbf{x} .
\end{aligned}
$$

Applying the first equality in (3.13) to $\xi\left(x^{*}, y^{*}, t^{n}\right)$, we bound the first term on the right-hand side of (5.4) by

$$
\begin{aligned}
& \left|\int_{\Omega_{1}} \xi\left(\mathbf{x}^{*}, t^{n}\right) \xi\left(x_{1}, y, t^{n+1}\right) d \mathbf{x}\right| \\
& =\left|\int_{y_{1}}^{d} \xi\left(x_{1}, y, t^{n+1}\right) \xi\left(x_{1}, y^{*}, t^{n}\right)\left[\int_{\tilde{a}(y)}^{x_{1}} \frac{x^{*}-a}{\Delta x} d x\right] d y\right| \\
& \leq \int_{y_{1}}^{d}\left[\frac{\Delta x}{2} K^{(3)}\left(x_{1}, y\right)+M \Delta t h\right]\left|\xi\left(x_{1}, y, t^{n+1}\right) \xi\left(x_{1}, y^{*}, t^{n}\right)\right| d y \\
& \leq \frac{\Delta x}{4} \int_{y_{1}}^{d} K^{(3)}\left(x_{1}, y\right) \xi^{2}\left(x_{1}, y, t^{n+1}\right) d y+\frac{\Delta x}{4} \int_{y_{1}}^{d} K^{(3)}\left(x_{1}, y\right) \xi^{2}\left(x_{1}, y^{*}, t^{n}\right) d y \\
& \quad+M \Delta t\left\|\xi\left(\mathbf{x}, t^{n+1}\right)\right\|_{L^{2}(\Omega)}^{2}+M \Delta t\left\|\xi\left(\mathbf{x}, t^{n}\right)\right\|_{L^{2}(\Omega)}^{2},
\end{aligned}
$$

where in the first " $\leq$ " sign of $(5.5)$, we have used the fact

$$
\begin{aligned}
\int_{\tilde{a}(y)}^{x_{1}} \frac{x^{*}-a}{\Delta x} d x & =\int_{\tilde{a}(y)}^{x_{1}}\left[\frac{x-V_{1}\left(x, y, t^{n+1}\right) \Delta t-a}{\Delta x}\right] d x \\
& =\int_{\tilde{a}(y)}^{x_{1}}\left[\frac{x-V_{1}\left(x_{1}, y, t^{n+1}\right) \Delta t-a}{\Delta x}+\mathcal{O}(\Delta t)\right] d x
\end{aligned}
$$




$$
\begin{aligned}
& \leq \frac{\Delta x}{2} K^{(3)}\left(x_{1}, y\right)-\frac{\left(\tilde{a}(y)-a-V_{1}\left(x_{1}, y, t^{n+1}\right) \Delta t\right)^{2}}{2 \Delta x}+M \Delta t h \\
& \leq \frac{\Delta x}{2} K^{(3)}\left(x_{1}, y\right)+M \Delta t h .
\end{aligned}
$$

At the second " $\leq$ " sign, we have used the equivalence between the discrete and continuous $L^{2}$ norms. Namely, there are two positive constants $M_{2}$ and $M_{3}$ such that

$$
M_{2}\left\|\xi\left(\mathbf{x}, t^{n+1}\right)\right\|_{L^{2}(\Omega)}^{2} \leq \sum_{i=1}^{I} \Delta x \int_{c}^{d} \xi^{2}\left(x_{i}, y, t^{n+1}\right) d y \leq M_{3}\left\|\xi\left(\mathbf{x}, t^{n+1}\right)\right\|_{L^{2}(\Omega)}^{2} .
$$

The second term on the right-hand side of (5.5) is rewritten as

$$
\begin{aligned}
& \frac{\Delta x}{4} \int_{y_{1}}^{d} K^{(3)}\left(x_{1}, y\right) \xi^{2}\left(x_{1}, y^{*}, t^{n}\right) d y \\
&=\frac{\Delta x}{4} \int_{y^{*}\left(\mathbf{x}_{1,1}\right)+\mathcal{O}(\Delta t \Delta x)}^{d^{*}\left(x_{1}, d\right)+\mathcal{O}(\Delta t \Delta x)}\left[K^{(3)}\left(x_{1}, z\right)+\mathcal{O}(\Delta t)\right] \xi^{2}\left(x_{1}, z, t^{n}\right) d z \\
& \leq \frac{\Delta x}{4} \int_{y^{*}\left(\mathbf{x}_{1,1}\right)}^{d^{*}\left(x_{1}, d\right)} K^{(3)}\left(x_{1}, y\right) \xi^{2}\left(x_{1}, y, t^{n}\right) d y+M \Delta t\left\|\xi\left(\mathbf{x}, t^{n}\right)\right\|_{L^{2}(\Omega)}^{2} \\
& \leq \frac{\Delta x}{4} \int_{y^{*}\left(\mathbf{x}_{1,1}\right)}^{y_{1}}\left[K^{(3)}\left(\mathbf{x}_{1,1}\right)+\mathcal{O}(h \Delta t)\right] \xi^{2}\left(\mathbf{x}_{1,1}, t^{n}\right) \frac{(y-c)^{2}}{(\Delta y)^{2}} d y \\
&+\frac{\Delta x}{4} \int_{y_{1}}^{d^{*}\left(x_{1}, d\right)} K^{(3)}\left(x_{1}, y\right) \xi^{2}\left(x_{1}, y, t^{n}\right) d y+M \Delta t\left\|\xi\left(\mathbf{x}, t^{n}\right)\right\|_{L^{2}(\Omega)}^{2} \\
&= \frac{\Delta x \Delta y}{12} K^{(3)}\left(\mathbf{x}_{1,1}\right)\left[1-\left(K^{(4)}\left(\mathbf{x}_{1,1}\right)\right)^{3 / 2}\right] \xi^{2}\left(\mathbf{x}_{1,1}, t^{n}\right) \\
&+\frac{\Delta x}{4} \int_{y_{1}}^{d^{*}\left(x_{1}, d\right)} K^{(3)}\left(x_{1}, y\right) \xi^{2}\left(x_{1}, y, t^{n}\right) d y+M \Delta t\left\|\xi\left(\mathbf{x}, t^{n}\right)\right\|_{L^{2}(\Omega)}^{2} .
\end{aligned}
$$

Combining (5.5) and (5.7) yields an upper bound for the first term on the righthand side of (5.4):

$$
\begin{aligned}
& \left|\int_{\Omega_{1}} \xi\left(\mathbf{x}^{*}, t^{n}\right) \xi\left(x_{1}, y, t^{n+1}\right) d \mathbf{x}\right| \\
& \leq \frac{\Delta x}{4} \int_{y_{1}}^{d} K^{(3)}\left(x_{1}, y\right) \xi^{2}\left(x_{1}, y, t^{n+1}\right) d y+\frac{\Delta x}{4} \int_{y_{1}}^{d^{*}\left(x_{1}, d\right)} K^{(3)}\left(x_{1}, y\right) \xi^{2}\left(x_{1}, y, t^{n}\right) d y \\
(5.8) & +\frac{\Delta x \Delta y}{12} K^{(3)}\left(\mathbf{x}_{1,1}\right)\left[1-\left(K^{(4)}\left(\mathbf{x}_{1,1}\right)\right)^{3 / 2}\right] \xi^{2}\left(\mathbf{x}_{1,1}, t^{n}\right) \\
& +M \Delta t\left\|\xi\left(\mathbf{x}, t^{n+1}\right)\right\|_{L^{2}(\Omega)}^{2}+M \Delta t\left\|\xi\left(\mathbf{x}, t^{n}\right)\right\|_{L^{2}(\Omega)}^{2} .
\end{aligned}
$$

By symmetry the third term on the right-hand side of (5.4) is bounded by

$$
\begin{aligned}
& \left|\int_{\Omega_{3}} \xi\left(\mathbf{x}^{*}, t^{n}\right) \xi\left(x, y_{1}, t^{n+1}\right) d \mathbf{x}\right| \\
& \leq \frac{\Delta y}{4} \int_{x_{1}}^{b} K^{(4)}\left(x, y_{1}\right) \xi^{2}\left(x, y_{1}, t^{n+1}\right) d x+\frac{\Delta y}{4} \int_{x_{1}}^{b^{*}\left(b, y_{1}\right)} K^{(4)}\left(x, y_{1}\right) \xi^{2}\left(x, y_{1}, t^{n}\right) d x \\
(5.9) & +\frac{\Delta x \Delta y}{12} K^{(4)}\left(\mathbf{x}_{1,1}\right)\left[1-\left(K^{(3)}\left(\mathbf{x}_{1,1}\right)\right)^{3 / 2}\right] \xi^{2}\left(\mathbf{x}_{1,1}, t^{n}\right) \\
& +M \Delta t\left\|\xi\left(\mathbf{x}, t^{n+1}\right)\right\|_{L^{2}(\Omega)}^{2}+M \Delta t\left\|\xi\left(\mathbf{x}, t^{n}\right)\right\|_{L^{2}(\Omega)}^{2} .
\end{aligned}
$$


Let

$$
\tilde{a}_{m}:=\min \tilde{a}(y) \quad \text { and } \quad \tilde{c}_{m}:=\min \tilde{c}(x) .
$$

The second term on the right-hand side of (5.4) is bounded similarly to (5.5):

$$
\begin{aligned}
\int_{\Omega_{2}} & \xi\left(\mathbf{x}^{*}, t^{n}\right) \xi\left(\mathbf{x}_{1,1}, t^{n+1}\right) d \mathbf{x} \\
\leq & \left|\xi\left(\mathbf{x}_{1,1}, t^{n+1}\right) \xi\left(\mathbf{x}_{1,1}, t^{n}\right)\right|\left[\int_{\tilde{a}_{m}}^{x_{1}} \frac{x^{*}-a}{\Delta x} d x\right]\left[\int_{\tilde{c}_{m}}^{y_{1}} \frac{y^{*}-c}{\Delta y} d y\right] \\
\leq & \frac{\Delta x \Delta y}{4} K^{(3)}\left(\mathbf{x}_{1,1}\right) K^{(4)}\left(\mathbf{x}_{1,1}\right)\left|\xi\left(\mathbf{x}_{1,1}, t^{n+1}\right) \xi\left(\mathbf{x}_{1,1}, t^{n}\right)\right| \\
& +M \Delta t\left\|\xi\left(\mathbf{x}, t^{n+1}\right)\right\|_{L^{2}(\Omega)}^{2}+M \Delta t\left\|\xi\left(\mathbf{x}, t^{n}\right)\right\|_{L^{2}(\Omega)}^{2} \\
\leq & \frac{\Delta x \Delta y}{8} K^{(3)}\left(\mathbf{x}_{1,1}\right) K^{(4)}\left(\mathbf{x}_{1,1}\right)\left[\xi^{2}\left(\mathbf{x}_{1,1}, t^{n+1}\right)+\xi^{2}\left(\mathbf{x}_{1,1}, t^{n}\right)\right] \\
& +M \Delta t\left\|\xi\left(\mathbf{x}, t^{n+1}\right)\right\|_{L^{2}(\Omega)}^{2}+M \Delta t\left\|\xi\left(\mathbf{x}, t^{n}\right)\right\|_{L^{2}(\Omega)}^{2} .
\end{aligned}
$$

We now estimate the last term on the right-hand side of (5.4) and obtain

$$
\begin{aligned}
& \left|\int_{\Omega_{4}} \xi\left(\mathbf{x}^{*}, t^{n}\right) \xi\left(\mathbf{x}, t^{n+1}\right) d \mathbf{x}\right| \\
& \quad \leq \frac{1}{2} \int_{\Omega_{4}} \xi^{2}\left(\mathbf{x}, t^{n+1}\right) d \mathbf{x}+\frac{1}{2} \int_{\Omega_{4}} \xi^{2}\left(\mathbf{x}^{*}, t^{n}\right) d \mathbf{x} \\
& \quad \leq \frac{1}{2} \int_{\Omega_{4}} \xi^{2}\left(\mathbf{x}, t^{n+1}\right) d \mathbf{x}+\frac{1}{2} \int_{\Omega_{4}^{*}} \xi^{2}\left(\mathbf{x}^{*}, t^{n}\right) \operatorname{det} \mathbf{J}^{-1}\left(t^{n} ; \mathbf{x}, t^{n+1}\right) d \mathbf{x}^{*} \\
& \quad \leq \frac{1}{2} \int_{\Omega_{4}} \xi^{2}\left(\mathbf{x}, t^{n+1}\right) d \mathbf{x}+\frac{1+M \Delta t}{2} \int_{\Omega_{4}^{*}} \xi^{2}\left(\mathbf{x}, t^{n}\right) d \mathbf{x},
\end{aligned}
$$

where $\Omega_{4}^{*}$ is the image of $\Omega_{4}$ under the transformation (2.6) with $\theta=t^{n}$ :

$$
\begin{aligned}
\int_{\Omega_{4}^{*}} \xi^{2}\left(\mathbf{x}, t^{n}\right) d \mathbf{x} & =\int_{\left(\Omega \backslash \Omega^{(O)}\left(t^{n}\right)\right) \cap\left(\left[x_{1}, b\right] \times\left[y_{1}, d\right]\right)} \xi^{2}\left(\mathbf{x}, t^{n}\right) d \mathbf{x} \\
& +\int_{y_{1}}^{d^{*}\left(x_{1}, d\right)}\left[\int_{x^{*}\left(x_{1}, y\right)+\mathcal{O}(\Delta t \Delta x)}^{x_{1}} \xi^{2}\left(\mathbf{x}, t^{n}\right) d x\right] d y \\
& +\int_{y^{*}\left(\mathbf{x}_{1,1}\right)}^{y_{1}}\left[\int_{x^{*}\left(x_{1}, y\right)+\mathcal{O}(\Delta t \Delta x)}^{x_{1}} \xi^{2}\left(\mathbf{x}, t^{n}\right) d x\right] d y \\
& +\int_{x_{1}}^{b^{*}\left(b, y_{1}\right)}\left[\int_{y^{*}\left(x, y_{1}\right)+\mathcal{O}(\Delta t \Delta x)}^{y_{1}} \xi^{2}\left(\mathbf{x}, t^{n}\right) d y\right] d x
\end{aligned}
$$

We use (3.13) and (5.6) to bound the second term on the right-hand side of (5.13):

$$
\begin{aligned}
& \int_{y_{1}}^{d^{*}\left(x_{1}, d\right)}\left[\int_{x^{*}\left(x_{1}, y\right)+\mathcal{O}(\Delta t \Delta x)}^{x_{1}} \xi^{2}\left(\mathbf{x}, t^{n}\right) d x\right] d y \\
& =\int_{y_{1}}^{d^{*}\left(x_{1}, d\right)} \xi^{2}\left(x_{1}, y, t^{n}\right)\left[\int_{x^{*}\left(x_{1}, y\right)}^{x_{1}} \frac{(x-a)^{2}}{(\Delta x)^{2}} d x+\mathcal{O}(\Delta t h)\right] d y \\
& =\frac{\Delta x}{3} \int_{y_{1}}^{d^{*}\left(x_{1}, d\right)}\left[1-\left(K^{(3)}\left(x_{1}, y\right)\right)^{3 / 2}\right] \xi^{2}\left(x_{1}, y, t^{n}\right) d y+M \Delta t\left\|\xi\left(\mathbf{x}, t^{n}\right)\right\|_{L^{2}(\Omega)}^{2} .
\end{aligned}
$$


Likewise, the last term on the right-hand side of (5.13) is bounded by

$$
\begin{aligned}
& \int_{x_{1}}^{b^{*}\left(b, y_{1}\right)}\left[\int_{y^{*}\left(x, y_{1}\right)+\mathcal{O}(\Delta t \Delta x)}^{y_{1}} \xi^{2}\left(\mathbf{x}, t^{n}\right) d y\right] d x \\
& \quad \leq \frac{\Delta y}{3} \int_{x_{1}}^{b^{*}\left(b, y_{1}\right)}\left[1-\left(K^{(4)}\left(x, y_{1}\right)\right)^{3 / 2}\right] \xi^{2}\left(x, y_{1}, t^{n}\right) d x+M \Delta t\left\|\xi\left(\mathbf{x}, t^{n}\right)\right\|_{L^{2}(\Omega)}^{2} .
\end{aligned}
$$

The third term on the right-hand side of (5.13) is controlled by

$$
\begin{aligned}
& \int_{y^{*}\left(\mathbf{x}_{1,1}\right)}^{y_{1}}\left[\int_{x^{*}\left(x_{1}, y\right)+\mathcal{O}(\Delta t \Delta x)}^{x_{1}} \xi^{2}\left(\mathbf{x}, t^{n}\right) d x\right] d y \\
& \leq \int_{y^{*}\left(\mathbf{x}_{1,1}\right)}^{y_{1}}\left[\int_{x^{*}\left(\mathbf{x}_{1,1}\right)}^{x_{1}} \xi^{2}\left(\mathbf{x}, t^{n}\right) d x\right] d y+M \Delta t\left\|\xi\left(\mathbf{x}, t^{n}\right)\right\|_{L^{2}(\Omega)}^{2} \\
& =\xi^{2}\left(\mathbf{x}_{1,1}, t^{n}\right)\left[\int_{x^{*}\left(\mathbf{x}_{1,1}\right)}^{x_{1}} \frac{(x-a)^{2}}{(\Delta x)^{2}} d x\right]\left[\int_{y^{*}\left(\mathbf{x}_{1,1}\right)}^{y_{1}} \frac{(y-c)^{2}}{(\Delta y)^{2}} d y\right] \\
& +M \Delta t\left\|\xi\left(\mathbf{x}, t^{n}\right)\right\|_{L^{2}(\Omega)}^{2} \\
& =\frac{\Delta x \Delta y}{9}\left[1-\left(K^{(3)}\left(\mathbf{x}_{1,1}\right)\right)^{3 / 2}\right]\left[1-\left(K^{(4)}\left(\mathbf{x}_{1,1}\right)\right)^{3 / 2}\right] \xi^{2}\left(\mathbf{x}_{1,1}, t^{n}\right) \\
& +M \Delta t\left\|\xi\left(\mathbf{x}, t^{n}\right)\right\|_{L^{2}(\Omega)}^{2} .
\end{aligned}
$$

Combining (5.12)-(5.14), we bound the last term on the right-hand side of (5.4) by

$$
\begin{aligned}
& \left|\int_{\Omega_{4}} \xi\left(\mathbf{x}^{*}, t^{n}\right) \xi\left(\mathbf{x}, t^{n+1}\right) d \mathbf{x}\right| \\
& \leq \frac{1}{2} \int_{\Omega_{4}} \xi^{2}\left(\mathbf{x}, t^{n+1}\right) d \mathbf{x}+\frac{1}{2} \int_{\left(\Omega \backslash \Omega^{(O)}\left(t^{n}\right)\right) \cap\left(\left[x_{1}, b\right] \times\left[y_{1}, d\right]\right)} \xi^{2}\left(\mathbf{x}, t^{n}\right) d \mathbf{x} \\
(5.15) & +\frac{\Delta x \Delta y}{18}\left[1-\left(K^{(3)}\left(\mathbf{x}_{1,1}\right)\right)^{3 / 2}\right]\left[1-\left(K^{(4)}\left(\mathbf{x}_{1,1}\right)\right)^{3 / 2}\right] \xi^{2}\left(\mathbf{x}_{1,1}, t^{n}\right) \\
& +\frac{\Delta x}{6} \int_{y_{1}}^{d^{*}\left(x_{1}, d\right)}\left[1-\left(K^{(3)}\left(x_{1}, y\right)\right)^{3 / 2}\right] \xi^{2}\left(x_{1}, y, t^{n}\right) d y \\
& +\frac{\Delta y}{6} \int_{x_{1}}^{b^{*}\left(b, y_{1}\right)}\left[1-\left(K^{(4)}\left(x, y_{1}\right)\right)^{3 / 2}\right] \xi^{2}\left(x, y_{1}, t^{n}\right) d x+M \Delta t\left\|\xi\left(\mathbf{x}, t^{n}\right)\right\|_{L^{2}(\Omega)}^{2} .
\end{aligned}
$$

Combining (5.4), (5.8), (5.9), (5.11), and (5.15), we obtain the estimate

$$
\begin{aligned}
& \left|\int_{\Omega \backslash \Omega^{(I)}\left(t^{n+1}\right)} \xi\left(\mathbf{x}^{*}, t^{n}\right) \hat{\xi}\left(\mathbf{x}, t^{n+1}\right) d \mathbf{x}\right| \\
& \leq \frac{1}{2} \int_{\Omega_{4}} \xi^{2}\left(\mathbf{x}, t^{n+1}\right) d \mathbf{x}+\frac{1}{2} \int_{\left(\Omega \backslash \Omega^{(O)}\left(t^{n}\right)\right) \cap\left(\left[x_{1}, b\right] \times\left[y_{1}, d\right]\right)} \xi^{2}\left(\mathbf{x}, t^{n}\right) d \mathbf{x} \\
& \quad+\frac{\Delta x}{4} \int_{y_{1}}^{d} K^{(3)}\left(x_{1}, y\right) \xi^{2}\left(x_{1}, y, t^{n+1}\right) d y+\frac{\Delta y}{4} \int_{x_{1}}^{b} K^{(4)}\left(x, y_{1}\right) \xi^{2}\left(x, y_{1}, t^{n+1}\right) d x \\
& \quad+\frac{\Delta x \Delta y}{8} K^{(3)}\left(\mathbf{x}_{1,1}\right) K^{(4)}\left(\mathbf{x}_{1,1}\right) \xi^{2}\left(\mathbf{x}_{1,1}, t^{n+1}\right)+M \Delta t\left\|\xi\left(\mathbf{x}, t^{n+1}\right)\right\|_{L^{2}(\Omega)}^{2} \\
& \quad+\frac{\Delta x}{4} \int_{y_{1}}^{d^{*}\left(x_{1}, d\right)}\left\{K^{(3)}\left(x_{1}, y\right)+\frac{2}{3}\left[1-\left(K^{(3)}\left(x_{1}, y\right)\right)^{3 / 2}\right]\right\} \xi^{2}\left(x_{1}, y, t^{n}\right) d y
\end{aligned}
$$




$$
\begin{aligned}
& +\frac{\Delta y}{4} \int_{x_{1}}^{b^{*}\left(b, y_{1}\right)}\left\{K^{(4)}\left(x, y_{1}\right)+\frac{2}{3}\left[1-\left(K^{(4)}\left(x, y_{1}\right)\right)^{3 / 2}\right]\right\} \xi^{2}\left(x, y_{1}, t^{n}\right) d x \\
& +\frac{\Delta x \Delta y}{8}\left\{K^{(3)}\left(\mathbf{x}_{1,1}\right) K^{(4)}\left(\mathbf{x}_{1,1}\right)+\frac{2}{3} K^{(3)}\left(\mathbf{x}_{1,1}\right)\left[1-\left(K^{(4)}\left(\mathbf{x}_{1,1}\right)\right)^{3 / 2}\right]\right. \\
(5.16)+ & +\frac{4}{9}\left[1-\left(K^{(3)}\left(\mathbf{x}_{1,1}\right)\right)^{3 / 2}\right]\left[1-\left(K^{(4)}\left(\mathbf{x}_{1,1}\right)\right)^{3 / 2}\right] \\
& \left.+\frac{2}{3} K^{(4)}\left(\mathbf{x}_{1,1}\right)\left[1-\left(K^{(3)}\left(\mathbf{x}_{1,1}\right)\right)^{3 / 2}\right]\right\} \xi^{2}\left(\mathbf{x}_{1,1}, t^{n}\right)+M \Delta t\left\|\xi\left(\mathbf{x}, t^{n}\right)\right\|_{L^{2}(\Omega)}^{2} \\
\leq & \frac{1}{2} \int_{\Omega_{4}} \xi^{2}\left(\mathbf{x}, t^{n+1}\right) d \mathbf{x}+\frac{1}{2} \int_{\left(\Omega \backslash \Omega^{(O)}\left(t^{n}\right)\right) \cap\left(\left[x_{1}, b\right] \times\left[y_{1}, d\right]\right)} \xi^{2}\left(\mathbf{x}, t^{n}\right) d \mathbf{x} \\
& +\frac{\Delta x}{4} \int_{y_{1}}^{d} K^{(3)}\left(x_{1}, y\right) \xi^{2}\left(x_{1}, y, t^{n+1}\right) d y+\frac{\Delta y}{4} \int_{x_{1}}^{b} K^{(4)}\left(x, y_{1}\right) \xi^{2}\left(x, y_{1}, t^{n+1}\right) d x \\
& +\frac{\Delta x}{4} \int_{y_{1}}^{d^{*}\left(x_{1}, d\right)} \xi^{2}\left(x_{1}, y, t^{n}\right) d y+\frac{\Delta y}{4} \int_{x_{1}}^{b^{*}\left(b, y_{1}\right)} \xi^{2}\left(x, y_{1}, t^{n}\right) d x \\
& +\frac{\Delta x \Delta y}{8} K^{(3)}\left(\mathbf{x}_{1,1}\right) K^{(4)}\left(\mathbf{x}_{1,1}\right) \xi^{2}\left(\mathbf{x}_{1,1}, t^{n+1}\right)+\frac{\Delta x \Delta y}{8} \xi^{2}\left(\mathbf{x}_{1,1}, t^{n}\right) \\
& +M \Delta t\left\|\xi\left(\mathbf{x}, t^{n+1}\right)\right\|_{L^{2}(\Omega)}^{2}+M \Delta t\left\|\xi\left(\mathbf{x}, t^{n}\right)\right\|_{L^{2}(\Omega)}^{2},
\end{aligned}
$$

where we have used the facts

$$
\begin{array}{rlrl}
\alpha & +\frac{2}{3}\left(1-\alpha^{3 / 2}\right) \leq 1 & & \forall \alpha \in[0,1], \\
\alpha \beta & +\frac{2}{3} \alpha\left(1-\beta^{3 / 2}\right)+\frac{2}{3} \beta\left(1-\alpha^{3 / 2}\right)+\frac{4}{9}\left(1-\alpha^{3 / 2}\right)\left(1-\beta^{3 / 2}\right) & \\
& =\left[\alpha+\frac{2}{3}\left(1-\alpha^{3 / 2}\right)\right]\left[\beta+\frac{2}{3}\left(1-\beta^{3 / 2}\right)\right] \leq 1 & \forall \alpha, \beta \in[0,1] .
\end{array}
$$

Case 2. $C r \geq 1$. If $V_{1}(\mathbf{x}, t)$ and $V_{2}(\mathbf{x}, t)$ are constant, then $C r \geq 1$ implies that $\Omega_{1}, \Omega_{2}$, and $\Omega_{3}$ in the decomposition (5.3) are empty sets $\emptyset$ and $\Omega \backslash \Omega^{(I)}\left(t^{n+1}\right)$ falls into the region $\left[x_{1}, b\right] \times\left[y_{1}, d\right]$. Namely,

$$
\Omega \backslash \Omega^{(I)}\left(t^{n+1}\right) \subset\left[x_{1}, b\right] \times\left[y_{1}, d\right], \quad \text { and } \quad \Omega_{1}=\Omega_{2}=\Omega_{3}=\emptyset .
$$

Nevertheless, (5.17) is not necessarily true when $V_{1}(\mathbf{x}, t)$ and $V_{2}(\mathbf{x}, t)$ are variables, because $\tilde{a}(y)$ and $\tilde{c}(x)$ defined by (5.2) can fall in any one of $\left[a, x_{1}\right] \times\left[y_{1}, d\right],\left[a, x_{1}\right] \times$ $\left[c, y_{1}\right],\left[x_{1}, b\right] \times\left[c, y_{1}\right]$, or $\left[x_{1}, b\right] \times\left[y_{1}, d\right]$ for different $x$ and $y$.

Here we first assume (5.17) in deriving an estimate and then extend the estimate to a general case. Under the assumption (5.17), (5.13) is now reduced to

$$
\begin{aligned}
& \int_{\Omega_{4}^{*}} \xi^{2}\left(\mathbf{x}, t^{n}\right) d \mathbf{x} \\
& \quad=\int_{\left(\Omega \backslash \Omega^{(O)}\left(t^{n}\right)\right) \cap\left(\left[x_{1}, b\right] \times\left[y_{1}, d\right]\right)} \xi^{2}\left(\mathbf{x}, t^{n}\right) d \mathbf{x}+\int_{c}^{y_{1}}\left[\int_{a}^{x_{1}} \xi^{2}\left(\mathbf{x}, t^{n}\right) d x\right] d y \\
& \quad+\int_{y_{1}}^{d^{*}\left(x_{1}, d\right)}\left[\int_{a}^{x_{1}} \xi^{2}\left(\mathbf{x}, t^{n}\right) d x\right] d y+\int_{x_{1}}^{b^{*}\left(b, y_{1}\right)}\left[\int_{c}^{y_{1}} \xi^{2}\left(\mathbf{x}, t^{n}\right) d y\right] d x
\end{aligned}
$$

The last two terms on the right-hand side of (5.18) are bounded by

$$
\int_{y_{1}}^{d^{*}\left(x_{1}, d\right.}\left[\int_{a}^{x_{1}} \xi^{2}\left(\mathbf{x}, t^{n}\right) d x\right] d y+\int_{x_{1}}^{b^{*}\left(b, y_{1}\right)}\left[\int_{c}^{y_{1}} \xi^{2}\left(\mathbf{x}, t^{n}\right) d y\right] d x
$$




$$
\begin{aligned}
= & \int_{y_{1}}^{d^{*}\left(x_{1}, d\right)} \xi^{2}\left(x_{1}, y, t^{n}\right)\left[\int_{a}^{x_{1}} \frac{(x-a)^{2}}{(\Delta x)^{2}} d x\right] d y \\
& +\int_{x_{1}}^{b^{*}\left(b, y_{1}\right)} \xi^{2}\left(x, y_{1}, t^{n}\right)\left[\int_{c}^{y_{1}} \frac{(y-c)^{2}}{(\Delta y)^{2}} d y\right] d x \\
= & \frac{\Delta x}{3} \int_{y_{1}}^{d^{*}\left(x_{1}, d\right)} \xi^{2}\left(x_{1}, y, t^{n}\right) d y+\frac{\Delta y}{3} \int_{x_{1}}^{b^{*}\left(b, y_{1}\right)} \xi^{2}\left(x, y_{1}, t^{n}\right) d x
\end{aligned}
$$

The second term on the right-hand side of (5.18) is bounded by

$$
\begin{aligned}
& \int_{c}^{y_{1}}\left[\int_{a}^{x_{1}} \xi^{2}\left(\mathbf{x}, t^{n}\right) d x\right] d y \\
& =\xi^{2}\left(\mathbf{x}_{1,1}, t^{n}\right)\left[\int_{a}^{x_{1}} \frac{(x-a)^{2}}{(\Delta x)^{2}} d x\right]\left[\int_{c}^{y_{1}} \frac{(y-c)^{2}}{(\Delta y)^{2}} d y\right]=\frac{\Delta x \Delta y}{9} \xi^{2}\left(\mathbf{x}_{1,1}, t^{n}\right)
\end{aligned}
$$

Therefore, (5.4) and (5.18)-(5.19) yield

$$
\begin{aligned}
& \left|\int_{\Omega \backslash \Omega^{(I)}\left(t^{n+1}\right)} \xi\left(\mathbf{x}^{*}, t^{n}\right) \hat{\xi}\left(\mathbf{x}, t^{n+1}\right) d \mathbf{x}\right| \\
& \leq \leq \frac{1}{2} \int_{\Omega_{4}} \xi^{2}\left(\mathbf{x}, t^{n+1}\right) d \mathbf{x}+\frac{1+M \Delta t}{2} \int_{\Omega_{4}^{*}} \xi^{2}\left(\mathbf{x}, t^{n}\right) d \mathbf{x} \\
& \leq \leq \frac{1}{2} \int_{\Omega_{4}} \xi^{2}\left(\mathbf{x}, t^{n+1}\right) d \mathbf{x}+\frac{1}{2} \int_{\left(\Omega \backslash \Omega^{(O)}\left(t^{n}\right)\right) \cap\left(\left[x_{1}, b\right] \times\left[y_{1}, d\right]\right)} \xi^{2}\left(\mathbf{x}, t^{n}\right) d \mathbf{x} \\
& \quad+\frac{\Delta x}{6} \int_{y_{1}}^{d^{*}\left(x_{1}, d\right)} \xi^{2}\left(x_{1}, y, t^{n}\right) d y+\frac{\Delta y}{6} \int_{x_{1}}^{b^{*}\left(b, y_{1}\right)} \xi^{2}\left(x, y_{1}, t^{n}\right) d x \\
& \quad+\frac{\Delta x \Delta y}{18} \xi^{2}\left(\mathbf{x}_{1,1}, t^{n}\right)+M \Delta t\left\|\xi\left(\mathbf{x}, t^{n}\right)\right\|_{L^{2}(\Omega)}^{2} .
\end{aligned}
$$

When $C r \geq 1$ and (5.17) is not valid, the upper bound for the left-hand side of (5.1) will be a combination of (5.16) and (5.20). Namely, if $\tilde{a}(y)$ or $\tilde{c}(x)$ falls in $\left[x_{1}, b\right] \times$ $\left[y_{1}, d\right]$ locally, then (5.20) holds. Otherwise, (5.16) holds but with a modification on the limits of the integrals in the third, fourth, and seventh terms on the right-hand side of (5.16). Combining all the cases leads to the estimate presented in (5.1).

6. Proof of Lemma 2. Notice that standard techniques only lead to the following suboptimal-order estimate for the second term on the right-hand side of (4.10):

$$
\begin{aligned}
& \left|\int_{\Omega^{(I)}\left(t^{n+1}\right)} \eta\left(\mathbf{x}, t^{n+1}\right) \hat{\xi}\left(\mathbf{x}, t^{n+1}\right) d \mathbf{x}\right| \\
& \quad \leq M\left\|\xi\left(\mathbf{x}, t^{n+1}\right)\right\|_{L^{2}\left(\Omega^{(I)}\left(t^{n+1}\right)\right)}\left\|\eta\left(\mathbf{x}, t^{n+1}\right)\right\|_{L^{2}\left(\Omega^{(I)}\left(t^{n+1}\right)\right)} \\
& \quad \leq M h^{2}\left\|\xi\left(\mathbf{x}, t^{n+1}\right)\right\|_{L^{2}\left(\Omega^{(I)}\left(t^{n+1}\right)\right)}\left\|u\left(\mathbf{x}, t^{n+1}\right)\right\|_{H^{2}\left(\Omega^{(I)}\left(t^{n+1}\right)\right)} \\
& \quad \leq M \Delta t\left\|\xi\left(\mathbf{x}, t^{n+1}\right)\right\|_{L^{2}\left(\Omega^{(I)}\left(t^{n+1}\right)\right)}^{2}+M\left[h^{4}+(\Delta t)^{2}\right]\left\|u\left(\mathbf{x}, t^{n+1}\right)\right\|_{W^{2, \infty}(\Omega)}^{2}
\end{aligned}
$$

To prove an optimal-order estimate for the scheme (2.21), we need one extra $\Delta t$ in the second term on the right-hand side of the above estimate. However, we cannot use the estimate

$$
\left\|\xi\left(\mathbf{x}, t^{n+1}\right)\right\|_{L^{2}\left(\Omega^{(I)}\left(t^{n+1}\right)\right)}^{2} \leq M \Delta t\left\|\xi\left(\mathbf{x}, t^{n+1}\right)\right\|_{L^{\infty}\left(\Omega^{(I)}\left(t^{n+1}\right)\right)}^{2},
$$


since the right-hand side is out of control. Hence, we have to prove the following superconvergence estimates.

LEMma 2. The following estimates hold for $u(\mathbf{x}, t) \in L^{\infty}\left(0, T ; H^{3}(\Omega)\right)$ :

$$
\begin{aligned}
& \left|\int_{\Omega^{(I)}\left(t^{n+1}\right)} \eta\left(\mathbf{x}, t^{n+1}\right) \hat{\xi}\left(\mathbf{x}, t^{n+1}\right) d \mathbf{x}\right| \\
& \leq \varepsilon \Delta x \int_{y_{1}}^{d} K^{(5)}\left(x_{1}, y\right) \xi^{2}\left(x_{1}, y, t^{n+1}\right) d y+\varepsilon \Delta y \int_{x_{1}}^{b} K^{(6)}\left(x, y_{1}\right) \xi^{2}\left(x, y_{1}, t^{n+1}\right) d x \\
& \quad+\varepsilon \Delta x \Delta y\left[K^{(5)}\left(\mathbf{x}_{1,1}\right)+K^{(6)}\left(\mathbf{x}_{1,1}\right) \Delta x\right] \xi^{2}\left(\mathbf{x}_{1,1}, t^{n+1}\right)+M \Delta t\left\|\xi\left(\mathbf{x}, t^{n+1}\right)\right\|_{L^{2}(\Omega)}^{2} \\
& \quad+M \Delta t\left[h^{4}+(\Delta t)^{2}\right]\left\|u\left(\mathbf{x}, t^{n+1}\right)\right\|_{H^{3}(\Omega)}^{2} \quad \text { for } \quad C r<1 \\
& \quad \int_{\Omega^{(O)}\left(t^{n}\right)} \eta^{2}\left(\mathbf{x}, t^{n}\right) d \mathbf{x} \\
& \quad \leq M(\Delta t)^{3}\left\|u\left(\mathbf{x}, t^{n}\right)\right\|_{H^{2}(\Omega)}^{2}+\lambda M \Delta t\left[h^{4}+(\Delta t)^{2}\right]\left\|u\left(\mathbf{x}, t^{n}\right)\right\|_{H^{3}(\Omega)}^{2}
\end{aligned}
$$

where $\lambda=1$ if $\mathrm{Cr}<1$ and 0 otherwise.

Proof. Using the $a_{m}$ and $c_{m}$ in (5.10), we write the left-hand side of (6.1) as

$$
\begin{aligned}
& \int_{\Omega^{(I)}\left(t^{n+1}\right)} \eta\left(\mathbf{x}, t^{n+1}\right) \hat{\xi}\left(\mathbf{x}, t^{n+1}\right) d \mathbf{x} \\
& =\sum_{j=2}^{J} \int_{y_{j-1}}^{y_{j}} \int_{a}^{\tilde{a}(y)} \eta\left(\mathbf{x}, t^{n+1}\right) \xi\left(x_{1}, y, t^{n+1}\right) d \mathbf{x} \\
(6.3) \quad & +\int_{c_{m}}^{y_{1}} \int_{a}^{\tilde{a}(y)} \eta\left(\mathbf{x}, t^{n+1}\right) \xi\left(\mathbf{x}_{1,1}, t^{n+1}\right) d \mathbf{x} \\
& +\sum_{i=2}^{I} \int_{x_{i-1}}^{x_{i}} \int_{c}^{\tilde{c}(x)} \eta\left(\mathbf{x}, t^{n+1}\right) \xi\left(x, y_{1}, t^{n+1}\right) d \mathbf{x} \\
& +\int_{a_{m}}^{x_{1}} \int_{c}^{\tilde{c}(x)} \eta\left(\mathbf{x}, t^{n+1}\right) \xi\left(\mathbf{x}_{1,1}, t^{n+1}\right) d \mathbf{x}+\int_{c}^{c_{m}} \int_{a}^{a_{m}} \eta\left(\mathbf{x}, t^{n+1}\right) \xi\left(\mathbf{x}_{1,1}, t^{n+1}\right) d \mathbf{x} .
\end{aligned}
$$

as

Using (3.9) with $m=2$, we rewrite the first term on the right-hand side of (6.3)

$$
\begin{aligned}
& \sum_{j=2}^{J} \int_{y_{j-1}}^{y_{j}} \int_{a}^{\tilde{a}(y)} \eta\left(\mathbf{x}, t^{n+1}\right) \xi\left(x_{1}, y, t^{n+1}\right) d \mathbf{x} \\
& =\sum_{j=2}^{J} \int_{y_{j-1}}^{y_{j}} \int_{a}^{\tilde{a}(y)} \xi\left(x_{1}, y, t^{n+1}\right)\left\{\int_{y_{j-1}}^{y_{j}} \int_{a}^{x_{1}} K_{1, j}^{(2,1)}(\alpha, \beta ; x, y) u_{\alpha \alpha \beta}\left(\alpha, \beta, t^{n+1}\right) d \alpha d \beta\right. \\
& \left.(6.4) \quad+\int_{a}^{x_{1}} K_{1}^{(2)}(\alpha ; x) u_{\alpha \alpha}\left(\alpha, y, t^{n+1}\right) d \alpha+\int_{y_{j-1}}^{y_{j}} K_{j}^{(2)}(\beta ; y) u_{\beta \beta}\left(x, \beta, t^{n+1}\right) d \beta\right\} d x d y .
\end{aligned}
$$

From definitions (3.4), (3.5), and (3.7), we have the following upper bounds:

$$
\begin{aligned}
K_{i, j}^{(2,1)}(\alpha, \beta ; x, y) & \leq \Delta x, & (x, y) & \in \bar{\Omega}_{i, j}, \\
K_{j}^{(2)}(\beta ; y) & \leq \Delta y, & y & \in\left[y_{j-1}, y_{j}\right], \\
K_{1}^{(2)}(\alpha ; x) & \leq M \Delta t, & x & \in[a, \tilde{a}(y)], \quad y \in\left[c_{m}, d\right] .
\end{aligned}
$$


Using (5.6), we bound the first term on the right-hand side of (6.4) by

$$
\begin{aligned}
& \left|\sum_{j=2}^{J} \int_{y_{j-1}}^{y_{j}} \int_{a}^{\tilde{a}(y)} \xi\left(x_{1}, y, t^{n+1}\right)\left[\int_{y_{j-1}}^{y_{j}} \int_{a}^{x_{1}} K_{1, j}^{(2,1)}(\alpha, \beta ; x, y) u_{\alpha \alpha \beta}\left(\alpha, \beta, t^{n+1}\right) d \alpha d \beta\right] d x d y\right| \\
& \leq \sum_{j=2}^{J} \Delta x \int_{y_{j-1}}^{y_{j}} \int_{a}^{\tilde{a}(y)}\left|\xi\left(x_{1}, y, t^{n+1}\right)\right|\left[\int_{y_{j-1}}^{y_{j}} \int_{a}^{x_{1}}\left|u_{\alpha \alpha \beta}\left(\alpha, \beta, t^{n+1}\right)\right| d \alpha d \beta\right] d x d y \\
& \leq M \Delta t h^{2} \sum_{j=2}^{J} \int_{y_{j-1}}^{y_{j}}\left|\xi\left(x_{1}, y, t^{n+1}\right)\right| d y\left[\int_{y_{j-1}}^{y_{j}} \int_{a}^{x_{1}} u_{\alpha \alpha \beta}^{2}\left(\alpha, \beta, t^{n+1}\right) d \alpha d \beta\right]^{1 / 2} \\
& \leq M \Delta t h^{5 / 2} \sum_{j=2}^{J}\left[\int_{y_{j-1}}^{y_{j}} \xi^{2}\left(x_{1}, y, t^{n+1}\right) d y\right]^{1 / 2}\left[\int_{y_{j-1}}^{y_{j}} \int_{a}^{x_{1}} u_{\alpha \alpha \beta}^{2}\left(\alpha, \beta, t^{n+1}\right) d \alpha d \beta\right]^{1 / 2} \\
& \leq M \Delta t\left\|\xi\left(\mathbf{x}, t^{n+1}\right)\right\|_{L^{2}(\Omega)}^{2}+M \Delta t h^{4}\left\|u\left(\mathbf{x}, t^{n+1}\right)\right\|_{H^{3}(\Omega)}^{2} .
\end{aligned}
$$

$$
\begin{aligned}
& \left|\sum_{j=2}^{J} \int_{y_{j-1}}^{y_{j}} \int_{a}^{\tilde{a}(y)} \xi\left(x_{1}, y, t^{n+1}\right)\left[\int_{a}^{x_{1}} K_{1}^{(2)}(\alpha ; x) u_{\alpha \alpha}\left(\alpha, y, t^{n+1}\right) d \alpha\right] d x d y\right| \\
& \quad \leq M \Delta t \sum_{j=2}^{J} \int_{y_{j-1}}^{y_{j}} \int_{a}^{\tilde{a}(y)}\left|\xi\left(x_{1}, y, t^{n+1}\right)\right|\left[\int_{a}^{x_{1}}\left|u_{\alpha \alpha}\left(\alpha, y, t^{n+1}\right)\right| d \alpha\right] d x d y \\
& \quad \leq C(\Delta t)^{2} \sum_{j=2}^{J} \int_{y_{j-1}}^{y_{j}}\left|\xi\left(x_{1}, y, t^{n+1}\right)\right|\left[\int_{a}^{x_{1}}\left|u_{\alpha \alpha}\left(\alpha, y, t^{n+1}\right)\right| d \alpha\right] d y \\
& \quad \leq M \Delta t\left\|\xi\left(\mathbf{x}, t^{n+1}\right)\right\|_{L^{2}(\Omega)}^{2}+C(\Delta t)^{3}\left\|u\left(\mathbf{x}, t^{n+1}\right)\right\|_{H^{2}(\Omega)}^{2}
\end{aligned}
$$

In $(6.5), K_{j}^{(2)}(\beta ; y)$ is bounded by $\Delta y$ instead of $\Delta t$. Therefore, the technique in deriving (6.7) cannot be used to bound the third term on the right-hand side of (6.4). We treat this term in a different way. Note that $u_{\beta \beta}\left(x, \beta, t^{n+1}\right) \in H^{1}(a, b)$. By Sobolev's embedding theorem, $u_{\beta \beta}\left(x, \beta, t^{n+1}\right)$ is continuous with respect to $x \in[a, b]$. Thus, there exists an $\bar{x} \in[a, b]$ such that

$$
u_{\beta \beta}\left(\bar{x}, \beta, t^{n+1}\right)=\frac{1}{b-a} \int_{a}^{b} u_{\beta \beta}\left(\alpha, \beta, t^{n+1}\right) d \alpha
$$

and

$$
\begin{aligned}
\left|u_{\beta \beta}\left(x, \beta, t^{n+1}\right)\right| & =\left|u_{\beta \beta}\left(\bar{x}, \beta, t^{n+1}\right)+\int_{\bar{x}}^{x} u_{\alpha \beta \beta}\left(\alpha, \beta, t^{n+1}\right) d \alpha\right| \\
& \leq \frac{1}{b-a} \int_{a}^{b}\left|u_{\beta \beta}\left(\alpha, \beta, t^{n+1}\right)\right| d \alpha+\int_{a}^{b}\left|u_{\alpha \beta \beta}\left(\alpha, \beta, t^{n+1}\right)\right| d \alpha
\end{aligned}
$$

Therefore, the third term on the right-hand side of (6.4) is bounded by

$$
\begin{aligned}
& \left|\sum_{j=2}^{J} \int_{y_{j-1}}^{y_{j}} \int_{a}^{\tilde{a}(y)} \xi\left(x_{1}, y, t^{n+1}\right)\left\{\int_{y_{j-1}}^{y_{j}} K_{j}^{(2)}(\beta ; y) u_{\beta \beta}\left(x, \beta, t^{n+1}\right) d \beta\right\} d x d y\right| \\
& \quad \leq M \Delta y \sum_{j=2}^{J} \int_{y_{j-1}}^{y_{j}} \int_{a}^{\tilde{a}(y)}\left|\xi\left(x_{1}, y, t^{n+1}\right)\right|\left[\int_{y_{j-1}}^{y_{j}}\left|u_{\beta \beta}\left(x, \beta, t^{n+1}\right)\right| d \beta\right] d x d y
\end{aligned}
$$


$\leq M \Delta t \Delta y \sum_{j=2}^{J}\left\{\int_{y_{j-1}}^{y_{j}}\left|V_{1}\left(\tilde{a}(y), y, t^{n+1}\right)\right|\left|\xi\left(x_{1}, y, t^{n+1}\right)\right| d y\right.$

$$
\left.\left[\int_{y_{j-1}}^{y_{j}} \int_{a}^{b}\left|u_{\beta \beta}\left(\alpha, \beta, t^{n+1}\right)\right| d \alpha d \beta+\int_{y_{j-1}}^{y_{j}} \int_{a}^{b}\left|u_{\alpha \beta \beta}\left(\alpha, \beta, t^{n+1}\right)\right| d \alpha d \beta\right]\right\}
$$

$$
\begin{aligned}
& \leq M \Delta t(\Delta y)^{2}\left[\int_{y_{1}}^{d}\left|V_{1}\left(x_{1}, y, t^{n+1}\right)+\mathcal{O}(\Delta x)\right| \xi^{2}\left(x_{1}, y, t^{n+1}\right) d y\right]^{1 / 2}\left\|u\left(\mathbf{x}, t^{n+1}\right)\right\|_{H^{3}(\Omega)} \\
& \leq \varepsilon \Delta x \int_{y_{1}}^{d} K^{(5)}\left(x_{1}, y\right) \xi^{2}\left(x_{1}, y, t^{n+1}\right) d y \\
& \quad+M \Delta t\left\|\xi\left(\mathbf{x}, t^{n+1}\right)\right\|_{L^{2}(\Omega)}^{2}+M \Delta t h^{4}\left\|u\left(\mathbf{x}, t^{n+1}\right)\right\|_{H^{3}(\Omega)}^{2},
\end{aligned}
$$

where we have used the fact that for $C r<1$, the $K^{(5)}(\mathbf{x})$ and $K^{(6)}(\mathbf{x})$ in (4.5) are reduced to

$$
K^{(5)}(\mathbf{x})=\frac{\left|V_{1}\left(\mathbf{x}, t^{n+1}\right)\right| \Delta t}{\Delta x}, \quad K^{(6)}(\mathbf{x})=\frac{\left|V_{2}\left(\mathbf{x}, t^{n+1}\right)\right| \Delta t}{\Delta y} .
$$

As in the estimate of (6.4), we use (3.9) to bound the second term on the righthand side of (6.3):

$$
\begin{aligned}
& \left|\int_{c_{m}}^{y_{1}} \int_{a}^{\tilde{a}(y)} \eta\left(\mathbf{x}, t^{n+1}\right) \xi\left(\mathbf{x}_{1,1}, t^{n+1}\right) d \mathbf{x}\right| \\
& =\mid \xi\left(\mathbf{x}_{1,1}, t^{n+1}\right) \int_{c_{m}}^{y_{1}} \int_{a}^{\tilde{a}(y)}\left[\int_{c}^{y_{1}} \int_{a}^{x_{1}} K_{1,1}^{(2,1)}(\alpha, \beta ; x, y) u_{\alpha \alpha \beta}\left(\alpha, \beta, t^{n+1}\right) d \alpha d \beta\right. \\
& \left.(6.11)+\int_{a}^{x_{1}} K_{1}^{(2)}(\alpha ; x) u_{\alpha \alpha}\left(\alpha, y, t^{n+1}\right) d \alpha+\int_{c}^{y_{1}} K_{1}^{(2)}(\beta ; y) u_{\beta \beta}\left(x, \beta, t^{n+1}\right) d \beta\right] d x d y \mid \\
& =\left|\xi\left(\mathbf{x}_{1,1}, t^{n+1}\right)\right| \int_{c_{m}}^{y_{1}} \int_{a}^{\tilde{a}(y)}\left[\Delta x \int_{c}^{y_{1}} \int_{a}^{x_{1}}\left|u_{\alpha \alpha \beta}\left(\alpha, \beta, t^{n+1}\right)\right| d \alpha d \beta\right. \\
& \left.\quad+\int_{a}^{x_{1}} M \Delta t\left|u_{\alpha \alpha}\left(\alpha, y, t^{n+1}\right)\right| d \alpha+\int_{c}^{y_{1}} \Delta y\left|u_{\beta \beta}\left(x, \beta, t^{n+1}\right)\right| d \beta\right] d x d y \\
& \leq \varepsilon \Delta x K^{(5)}\left(\mathbf{x}_{1,1}\right) \xi^{2}\left(\mathbf{x}_{1,1}, t^{n+1}\right)+M \Delta t\left\|\xi\left(\mathbf{x}, t^{n+1}\right)\right\|_{L^{2}(\Omega)}^{2} \\
& \quad+M \Delta t\left[h^{4}+(\Delta t)^{2}\right]\left\|u\left(\mathbf{x}, t^{n+1}\right)\right\|_{H^{3}(\Omega)}^{2},
\end{aligned}
$$

where we have used (5.6) and (6.9).

Combining (6.4), (6.6), (6.7), (6.10), and (6.11) yields an upper bound for the first two terms on the right-hand side of (6.3). The remaining terms can be bounded similarly. Thus, we have proven (6.1).

We now turn to $(6.2)$ and first prove it for $C r<1$. We rewrite this term as

$$
\int_{\Omega^{(O)}\left(t^{n}\right)} \eta^{2}\left(\mathbf{x}, t^{n}\right) d \mathbf{x}=\int_{c}^{d} \int_{b^{*}(b, y)}^{b} \eta^{2}\left(\mathbf{x}, t^{n}\right) d x d y+\int_{a}^{b} \int_{d^{*}(x, d)}^{d} \eta^{2}\left(\mathbf{x}, t^{n}\right) d y d x
$$

Using (6.5) and the fact

$$
K_{I}^{(2)}(\alpha ; x) \leq M \Delta t, \quad x \in\left[b^{*}(b, y), b\right], \quad y \in[c, d]
$$


we bound the first term on the right-hand side of (6.1) by

$$
\begin{aligned}
& \int_{c}^{d} \int_{b^{*}(b, y)}^{b} \eta^{2}\left(\mathbf{x}, t^{n}\right) d x d y \\
& =\sum_{j=1}^{J} \int_{y_{j-1}}^{y_{j}} \int_{b^{*}(b, y)}^{b}\left\{\int_{y_{j-1}}^{y_{j}} \int_{x_{I-1}}^{b} K_{I, j}^{(2,1)}(\alpha, \beta ; x, y) u_{\alpha \alpha \beta}\left(\alpha, \beta, t^{n+1}\right) d \alpha d \beta\right. \\
& \left.+\int_{x_{I-1}}^{b} K_{I}^{(2)}(\alpha ; x) u_{\alpha \alpha}\left(\alpha, y, t^{n+1}\right) d \alpha+\int_{y_{j-1}}^{y_{j}} K_{j}^{(2)}(\beta ; y) u_{\beta \beta}\left(x, \beta, t^{n+1}\right) d \beta\right\}^{2} d x d y \\
& \leq M \sum_{j=1}^{J} \int_{y_{j-1}}^{y_{j}}\left\{\Delta t(\Delta x)^{3} \Delta y \int_{y_{j-1}}^{y_{j}} \int_{x_{I-1}}^{b} u_{\alpha \alpha \beta}^{2}\left(\alpha, \beta, t^{n+1}\right) d \alpha d \beta\right. \\
& +(\Delta t)^{3} \Delta x \int_{x_{I-1}}^{b} u_{\alpha \alpha}^{2}\left(\alpha, y, t^{n+1}\right) d \alpha+\Delta t(\Delta y)^{3} \int_{y_{j-1}}^{y_{j}}\left[\int_{a}^{b} u_{\beta \beta}^{2}\left(\alpha, \beta, t^{n+1}\right) d \alpha\right. \\
& \left.\left.+\int_{a}^{b} u_{\alpha \beta \beta}^{2}\left(\alpha, \beta, t^{n+1}\right) d \alpha\right] d \beta\right\}^{2} d x d y \\
& \leq M \Delta t\left[h^{4}+(\Delta t)^{2}\right]\left\|u\left(\mathbf{x}, t^{n+1}\right)\right\|_{H^{3}(\Omega)}^{2},
\end{aligned}
$$

where we have used (6.9) in estimating the $u_{\beta \beta}$ term.

When $C r \geq 1, h \leq M \Delta t$. Hence, we have

$$
\int_{\Omega^{(O)}\left(t^{n}\right)} \eta^{2}\left(\mathbf{x}, t^{n}\right) d \mathbf{x} \leq M h^{4}\left\|u\left(\mathbf{x}, t^{n}\right)\right\|_{H^{2}(\Omega)}^{2} \leq M(\Delta t)^{4}\left\|u\left(\mathbf{x}, t^{n}\right)\right\|_{H^{2}(\Omega)}^{2} .
$$

By symmetry, we can also obtain the same upper bound for the second term on the right-hand side of (6.12). Thus, we have proven (6.2).

7. Proof of Lemma 3. Standard techniques only generate the suboptimal-order estimate

$$
\begin{aligned}
& \left|\int_{\Omega \backslash \Omega^{(I)}\left(t^{n+1}\right)}\left[\eta\left(\mathbf{x}, t^{n}\right)-\eta\left(\mathbf{x}^{*}, t^{n}\right)\right] \hat{\xi}\left(\mathbf{x}, t^{n+1}\right) d \mathbf{x}\right| \\
& \quad \leq M \Delta t\left\|\xi\left(\mathbf{x}, t^{n+1}\right)\right\|_{L^{2}(\Omega)}^{2}+M \Delta t h^{2}\left\|u\left(\mathbf{x}, t^{n+1}\right)\right\|_{H^{2}(\Omega)}^{2},
\end{aligned}
$$

which then leads to a suboptimal-order estimate of the scheme (2.21) and does not reflect the strength of the ELLAM scheme. In this section we prove the following superconvergence estimate.

Lemma 3. If $C r<1$, the following estimate holds for $u(\mathbf{x}, t) \in L^{\infty}\left(0, T ; H^{3}(\Omega)\right)$ :

$$
\begin{aligned}
&\left|\int_{\Omega \backslash \Omega^{(I)}\left(t^{n+1}\right)}\left[\eta\left(\mathbf{x}, t^{n}\right)-\eta\left(\mathbf{x}^{*}, t^{n}\right)\right] \hat{\xi}\left(\mathbf{x}, t^{n+1}\right) d \mathbf{x}\right| \\
& \leq \varepsilon \int_{S_{n}^{(O)}} \mathbf{v} \cdot \mathbf{n} \xi^{2}(\mathbf{x}, t) d S+\varepsilon \Delta x \Delta y\left[K^{(5)}\left(\mathbf{x}_{1,1}\right)+K^{(6)}\left(\mathbf{x}_{1,1}\right)\right] \xi^{2}\left(\mathbf{x}_{1,1}, t^{n+1}\right) \\
&(7.1) \quad+\varepsilon \Delta x \int_{y_{1}}^{d} K^{(5)}\left(x_{1}, y\right) \xi^{2}\left(x_{1}, y, t^{n+1}\right) d y+\varepsilon \Delta y \int_{x_{1}}^{b} K^{(6)}\left(x, y_{1}\right) \xi^{2}\left(x, y_{1}, t^{n+1}\right) d x \\
& \quad+M \Delta t\left\|\xi\left(\mathbf{x}, t^{n+1}\right)\right\|_{L^{2}(\Omega)}^{2}+M \Delta t\left[h^{4}+(\Delta t)^{2}\right]\left\|u\left(\mathbf{x}, t^{n+1}\right)\right\|_{H^{3}(\Omega)}^{2},
\end{aligned}
$$


where $K^{(5)}(\mathbf{x})$ and $K^{(6)}(\mathbf{x})$ are defined in (4.5).

Proof. The left-hand side of (7.1) can be rewritten

$$
\begin{aligned}
& \int_{\Omega \backslash \Omega^{(I)}\left(t^{n+1}\right)}\left[\eta\left(\mathbf{x}, t^{n}\right)-\eta\left(\mathbf{x}^{*}, t^{n}\right)\right] \hat{\xi}\left(\mathbf{x}, t^{n+1}\right) d \mathbf{x} \\
& =\int_{\Omega \backslash \Omega^{(I)}\left(t^{n+1)}\right)}\left[\eta\left(x, y^{*}, t^{n}\right)-\eta\left(\mathbf{x}^{*}, t^{n}\right)\right] \hat{\xi}\left(\mathbf{x}, t^{n+1}\right) d \mathbf{x} \\
& \quad+\int_{\Omega \backslash \Omega^{(I)}\left(t^{n+1}\right)}\left[\eta\left(\mathbf{x}, t^{n}\right)-\eta\left(x, y^{*}, t^{n}\right)\right] \hat{\xi}\left(\mathbf{x}, t^{n+1}\right) d \mathbf{x} .
\end{aligned}
$$

We substitute the following expression into the second term on the right-hand side of (7.2):

$$
\begin{aligned}
\eta\left(x, y^{*}, t^{n}\right)-\eta\left(x^{*}, y^{*}, t^{n}\right) & =\int_{0}^{1} \frac{d}{d \theta} \eta\left(x^{*}+\theta\left(x-x^{*}\right), y^{*}, t^{n}\right) d \theta \\
& =\int_{0}^{1} \eta_{x}\left(x^{*}+\theta\left(x-x^{*}\right), y^{*}, t^{n}\right)\left(x-x^{*}\right) d \theta
\end{aligned}
$$

Then we integrate the resulting term by parts with respect to $x$ :

$$
\begin{aligned}
& \int_{\Omega \backslash \Omega^{(I)}\left(t^{n+1}\right)}\left[\eta\left(x, y, t^{n}\right)-\eta\left(x, y^{*}, t^{n}\right)\right] \hat{\xi}\left(x, y, t^{n+1}\right) d x d y \\
&=-\int_{0}^{1} \int_{\Omega \backslash \Omega^{(I)}\left(t^{n+1}\right)}\left[\eta\left(x^{*}+\theta\left(x-x^{*}\right), y^{*}, t^{n}\right)\left(x-x^{*}\right)_{x}\right] \hat{\xi}\left(x, y, t^{n+1}\right) d x d y d \theta \\
&(7.3) \quad-\int_{0}^{1} \int_{\Omega \backslash \Omega^{(I)}\left(t^{n+1}\right)}\left[\eta{ }_{y}\left(x^{*}+\theta\left(x-x^{*}\right), y^{*}, t^{n}\right) y_{y}^{*}\left(x-x^{*}\right)\right] \hat{\xi}\left(x, y, t^{n+1}\right) d x d y d \theta \\
& \quad-\int_{0}^{1} \int_{\Omega \backslash \Omega^{(I)}\left(t^{n+1}\right)}\left[\eta\left(x^{*}+\theta\left(x-x^{*}\right), y^{*}, t^{n}\right)\left(x-x^{*}\right)\right] \hat{\xi}_{x}\left(x, y, t^{n+1}\right) d x d y d \theta \\
& \quad+\int_{0}^{1} \int_{\tilde{c}(b)}^{d}\left[\eta\left(b^{*}+\theta\left(b-b^{*}\right), y^{*}, t^{n}\right)\left(b-b^{*}\right)\right] \xi\left(b, y, t^{n+1}\right) d y d \theta \\
&-\int_{0}^{1} \int_{\tilde{c}}^{d}\left[\eta\left(a+\theta(\tilde{a}(y)-a), y^{*}, t^{n}\right)(\tilde{a}-a)\right] \xi\left(x_{1}, y, t^{n+1}\right) d y d \theta,
\end{aligned}
$$

where in the last term we have used the second and fourth equalities in (3.13).

Using (4.6) and the fact that

$$
\left(x-x^{*}\right)_{x}=V_{1 x}\left(x, y, t^{n+1}\right) \Delta t, \quad y_{x}^{*}=-V_{2 x}\left(x, y, t^{n+1}\right) \Delta t,
$$

the first and second terms on the right-hand side of (7.3) are bounded by

$$
\begin{aligned}
& \mid \int_{0}^{1} \int_{\Omega \backslash \Omega^{(I)}\left(t^{n+1}\right)}\left[\eta\left(x^{*}+\theta\left(x-x^{*}\right), y^{*}, t^{n}\right)\left(x-x^{*}\right)_{x}\right] \hat{\xi}\left(x, y, t^{n+1}\right) d x d y d \theta \\
(7.4) & -\int_{0}^{1} \int_{\Omega \backslash \Omega^{(I)}\left(t^{n+1}\right)}\left[\eta_{y}\left(x^{*}+\theta\left(x-x^{*}\right), y^{*}, t^{n}\right) y_{y}^{*}\left(x-x^{*}\right)\right] \hat{\xi}\left(x, y, t^{n+1}\right) d x d y d \theta \mid \\
& \leq M \Delta t\left\|\hat{\xi}\left(\mathbf{x}, t^{n+1}\right)\right\|_{L^{2}(\Omega)}\left(\left\|\eta\left(\mathbf{x}, t^{n}\right)\right\|_{L^{2}(\Omega)}+\Delta t\left\|\eta_{x}\left(\mathbf{x}, t^{n}\right)\right\|_{L^{2}(\Omega)}\right) \\
& \leq M \Delta t\left\|\xi\left(\mathbf{x}, t^{n+1}\right)\right\|_{L^{2}(\Omega)}^{2}+M \Delta t\left(h^{4}+(\Delta t)^{2} h^{2}\right)\left\|u\left(\mathbf{x}, t^{n}\right)\right\|_{H^{2}(\Omega)}^{2} .
\end{aligned}
$$


For $C r<1, t_{n, I C-1}=t^{n+1}$. The last equality in (3.13) gives $\xi(\mathbf{x}, t)=\xi\left(\mathbf{x}, t^{n+1}\right)$ for $t \in\left[t^{n}, t^{n+1}\right]$. The fourth term on the right-hand side of (7.3) is bounded by

$$
\begin{aligned}
\left|\int_{0}^{1} \int_{\tilde{c}(b)}^{d}\left[\eta\left(b^{*}+\theta\left(b-b^{*}\right), y^{*}, t^{n}\right)\left(b-b^{*}\right)\right] \xi\left(b, y, t^{n+1}\right) d y d \theta\right| \\
=\Delta t\left|\int_{0}^{1} \int_{\tilde{c}(b)}^{d} \eta\left(b^{*}+\theta\left(b-b^{*}\right), y^{*}, t^{n}\right) V_{1}\left(b, y, t^{n+1}\right) \xi\left(b, y, t^{n+1}\right) d y d \theta\right| \\
=\Delta t \mid \int_{\tilde{c}(b)}^{d} \eta\left(b, y^{*}, t^{n}\right) V_{1}\left(b, y, t^{n+1}\right) \xi\left(b, y, t^{n+1}\right) d y d \theta \\
\quad-\int_{0}^{1} \int_{\tilde{c}(b)}^{d}\left[\int_{b^{*}+\theta\left(b-b^{*}\right)}^{b} \eta_{z}\left(z, y^{*}, t^{n}\right) d z\right] V_{1}\left(b, y, t^{n+1}\right) \xi\left(b, y, t^{n+1}\right) d y d \theta \mid \\
\leq M \Delta t\left[\int_{c}^{d} V_{1}\left(b, y, t^{n+1}\right) \xi^{2}\left(b, y, t^{n+1}\right) d y\right]^{1 / 2}\left[\left\|\eta\left(b, y, t^{n}\right)\right\|_{L^{2}(c, d)}\right. \\
\left.\quad+(\Delta t)^{1 / 2}\left\|\eta_{x}\left(\mathbf{x}, t^{n}\right)\right\|_{L^{2}(\Omega)}\right] \\
\leq \varepsilon \int_{t^{n}}^{t^{n+1}} \int_{c}^{d} \mathbf{v} \cdot \mathbf{n} \xi^{2}(\mathbf{x}, t) d S+M \Delta t\left[h^{4}\left\|u\left(b, y, t^{n}\right)\right\|_{H^{2}(c, d)}^{2}\right. \\
\left.\quad+\Delta t h^{2}\left\|u\left(\mathbf{x}, t^{n}\right)\right\|_{H^{2}(\Omega)}^{2}\right] \\
\leq \varepsilon \int_{t^{n}}^{t^{n+1}} \int_{c}^{d} \mathbf{v} \cdot \mathbf{n} \xi^{2}(\mathbf{x}, t) d S+M \Delta t\left[h^{4}+(\Delta t)^{2}\right]\left\|u\left(\mathbf{x}, t^{n}\right)\right\|_{H^{3}(\Omega)}^{2},
\end{aligned}
$$

where, at the last step, we have used the trace theorem (4.13).

Since $V_{1}\left(\tilde{a}(y), y, t^{n+1}\right)-V_{1}\left(x_{1}, y, t^{n+1}\right)=\mathcal{O}(\Delta x)$, we can bound the last term on the right-hand side of (7.3) similarly. Noting that $V_{1}\left(\tilde{a}(y), y, t^{n+1}\right)-V_{1}\left(x_{1}, y, t^{n+1}\right)=$ $\mathcal{O}(\Delta x)$, we have

$$
\begin{aligned}
& \left|\int_{0}^{1} \int_{\tilde{c}}^{d}\left[\eta\left(a+\theta(\tilde{a}(y)-a), y^{*}, t^{n}\right)(\tilde{a}-a)\right] \xi\left(x_{1}, y, t^{n+1}\right) d y d \theta\right| \\
& =\Delta t \mid \int_{\tilde{c}}^{d}\left[\eta\left(a, y^{*}, t^{n}\right)+\int_{0}^{1} \int_{a}^{a+\theta(\tilde{a}(y)-a)} \eta_{z}\left(z, y^{*}, t^{n}\right) d z d \theta\right] \\
& \quad\left[V_{1}\left(x_{1}, y, t^{n+1}\right)+\left(V_{1}\left(\tilde{a}(y), y, t^{n+1}\right)-V_{1}\left(x_{1}, y, t^{n+1}\right)\right)\right] \xi\left(x_{1}, y, t^{n+1}\right) d y \mid \\
& \leq \varepsilon \Delta x \int_{y_{1}}^{d} K^{(5)}\left(x_{1}, y\right) \xi^{2}\left(x_{1}, y, t^{n+1}\right) d y+\varepsilon \Delta x \Delta y K^{(5)}\left(\mathbf{x}_{1,1}\right) \xi^{2}\left(\mathbf{x}_{1,1}, t^{n+1}\right) \\
& \quad+M \Delta t\left\|\xi\left(\mathbf{x}, t^{n+1}\right)\right\|_{L^{2}(\Omega)}^{2}+M \Delta t\left[h^{4}\left\|u\left(a, y, t^{n}\right)\right\|_{H^{2}(c, d)}^{2}+\Delta t h^{2}\left\|u\left(\mathbf{x}, t^{n}\right)\right\|_{H^{2}(\Omega)}^{2}\right] \\
& \leq \varepsilon \Delta x \int_{y_{1}}^{d} K^{(5)}\left(x_{1}, y\right) \xi^{2}\left(x_{1}, y, t^{n+1}\right) d y+\varepsilon \Delta x \Delta y K^{(5)}\left(\mathbf{x}_{1,1}\right) \xi^{2}\left(\mathbf{x}_{1,1}, t^{n+1}\right) \\
& \quad+M \Delta t\left\|\xi\left(\mathbf{x}, t^{n+1}\right)\right\|_{L^{2}(\Omega)}^{2}+M \Delta t\left[h^{4}+(\Delta t)^{2}\right]\left\|u\left(\mathbf{x}, t^{n}\right)\right\|_{H^{3}(\Omega)}^{2},
\end{aligned}
$$

where at the first " $\leq$ " sign we have used (5.6) and at the second " $\leq$ " sign we have used the trace theorem (4.13). 
The second and fourth equalities in (3.13) imply $\hat{\xi}_{x}\left(x, y, t^{n+1}\right)=0$ for $x \in\left[a, x_{1}\right]$. Hence,

$$
\begin{aligned}
\int_{0}^{1} \int_{\Omega \backslash \Omega^{(I)}\left(t^{n+1}\right)}\left[\eta\left(x^{*}+\theta\left(x-x^{*}\right), y^{*}, t^{n}\right)\left(x-x^{*}\right)\right] \hat{\xi}_{x}\left(\mathbf{x}, t^{n+1}\right) d \mathbf{x} d \theta \\
=\Delta t \int_{0}^{1} \int_{x_{1}}^{b} \int_{\tilde{c}(x)}^{d} \eta\left(x^{*}+\theta\left(x-x^{*}\right), y^{*}, t^{n}\right) V_{1}\left(\mathbf{x}, t^{n+1}\right) \xi_{x}\left(\mathbf{x}, t^{n+1}\right) d \mathbf{x} d \theta \\
=\Delta t \int_{x_{1}}^{b} \int_{\tilde{c}(x)}^{d} V_{1}\left(\mathbf{x}, t^{n+1}\right) \xi_{x}\left(\mathbf{x}, t^{n+1}\right)\left[\eta\left(\mathbf{x}, t^{n}\right)\right. \\
\left.\quad+\int_{0}^{1} \int_{0}^{1} \frac{d}{d \gamma} \eta\left(x+\gamma(1-\theta)\left(x^{*}-x\right), y+\gamma\left(y^{*}-y\right), t^{n}\right) d \gamma d \theta\right] d \mathbf{x} \\
=\Delta t \int_{x_{1}}^{b} \int_{c}^{d} V_{1}\left(\mathbf{x}, t^{n+1}\right) \xi_{x}\left(\mathbf{x}, t^{n+1}\right) \eta\left(\mathbf{x}, t^{n}\right) d \mathbf{x} \\
-\Delta t \int_{x_{1}}^{b} \int_{c}^{\tilde{c}(x)} V_{1}\left(\mathbf{x}, t^{n+1}\right) \xi_{x}\left(\mathbf{x}, t^{n+1}\right) \eta\left(\mathbf{x}, t^{n}\right) d \mathbf{x} \\
+\Delta t \int_{0}^{1} \int_{0}^{1} \int_{x_{1}}^{b} \int_{\tilde{c}(x)}^{d} V_{1}\left(\mathbf{x}, t^{n+1}\right) \xi_{x}\left(\mathbf{x}, t^{n+1}\right) \\
\quad\left[(1-\theta)\left(x^{*}-x\right) \eta_{x}\left(x+\gamma(1-\theta)\left(x^{*}-x\right), y+\gamma\left(y^{*}-y\right), t^{n}\right)\right. \\
\left.\quad+\left(y^{*}-y\right) \eta_{y}\left(x+\gamma(1-\theta)\left(x^{*}-x\right), y+\gamma\left(y^{*}-y\right), t^{n}\right)\right] d \mathbf{x} d \gamma d \theta
\end{aligned}
$$

Using the inverse inequality in (3.2), we bound the third term on the right-hand side of (7.5) by

$$
\begin{aligned}
\mid \Delta t & \int_{0}^{1} \int_{0}^{1} \int_{x_{1}}^{b} \int_{\tilde{c}(x)}^{d} V_{1}\left(\mathbf{x}, t^{n+1}\right) \xi_{x}\left(\mathbf{x}, t^{n+1}\right) \\
& \quad\left[(1-\theta)\left(x^{*}-x\right) \eta_{x}\left(x+\gamma(1-\theta)\left(x^{*}-x\right), y+\gamma\left(y^{*}-y\right), t^{n}\right)\right. \\
& \left.+\left(y^{*}-y\right) \eta_{y}\left(x+\gamma(1-\theta)\left(x^{*}-x\right), y+\gamma\left(y^{*}-y\right), t^{n}\right)\right] d \mathbf{x} d \gamma d \theta \mid \\
\leq & M(\Delta t)^{2} h\left\|\xi_{x}\left(\mathbf{x}, t^{n+1}\right)\right\|_{L^{2}(\Omega)}\left\|u\left(\mathbf{x}, t^{n}\right)\right\|_{H^{2}(\Omega)} \\
\leq & M \Delta t\left\|\xi\left(\mathbf{x}, t^{n+1}\right)\right\|_{L^{2}(\Omega)}^{2}+M(\Delta t)^{3}\left\|u\left(\mathbf{x}, t^{n}\right)\right\|_{H^{2}(\Omega)}^{2} .
\end{aligned}
$$

Recalling the first equality in (3.13), we have $\xi_{x}\left(x, c, t^{n+1}\right)=0$. Using the inverse inequality in (3.2) and the fact that $\Delta t \leq M \Delta x$ for $C r<1$, we bound the second term on the right-hand side of (7.5) by

$$
\begin{aligned}
& \left|\Delta t \int_{x_{1}}^{b} \int_{c}^{\tilde{c}(x)} \eta\left(\mathbf{x}, t^{n}\right) V_{1}\left(\mathbf{x}, t^{n+1}\right) \xi_{x}\left(\mathbf{x}, t^{n+1}\right) d \mathbf{x}\right| \\
& \leq M \Delta t\left[\int_{x_{1}}^{b} \int_{c}^{\tilde{c}(x)} \xi_{x}^{2}\left(x, y_{1}, t^{n+1}\right) \frac{(y-c)^{2}}{(\Delta y)^{2}} d y d x\right]^{1 / 2}\left\|\eta\left(\mathbf{x}, t^{n}\right)\right\|_{L^{2}(\Omega)} \\
& \leq M(\Delta t)^{5 / 2}(\Delta y)^{-1} h^{2}\left[\int_{x_{1}}^{b} \xi_{x}^{2}\left(x, y_{1}, t^{n+1}\right) d x\right]^{1 / 2}\left\|u\left(\mathbf{x}, t^{n}\right)\right\|_{H^{2}(\Omega)}
\end{aligned}
$$




$$
\begin{aligned}
& \leq M(\Delta t)^{2}\left\|\xi\left(\mathbf{x}, t^{n+1}\right)\right\|_{L^{2}(\Omega)}\left\|u\left(\mathbf{x}, t^{n}\right)\right\|_{H^{2}(\Omega)} \\
& \leq M \Delta t\left\|\xi\left(\mathbf{x}, t^{n+1}\right)\right\|_{L^{2}(\Omega)}^{2}+M(\Delta t)^{3}\left\|u\left(\mathbf{x}, t^{n}\right)\right\|_{H^{2}(\Omega)}^{2}
\end{aligned}
$$

We sum the first term on the right-hand side of (7.5) by parts and obtain

$$
\begin{aligned}
\Delta t & \int_{x_{1}}^{b} \int_{c}^{d} V_{1}\left(\mathbf{x}, t^{n+1}\right) \eta\left(\mathbf{x}, t^{n}\right) \xi_{x}\left(\mathbf{x}, t^{n+1}\right) d \mathbf{x} \\
= & \frac{\Delta t}{\Delta x} \sum_{i=2}^{I} \int_{x_{i-1}}^{x_{i}} \int_{c}^{d} V_{1}\left(\mathbf{x}, t^{n+1}\right) \eta\left(\mathbf{x}, t^{n}\right)\left[\xi\left(x_{i}, y, t^{n+1}\right)-\xi\left(x_{i-1}, y, t^{n+1}\right)\right] d \mathbf{x} \\
= & \frac{\Delta t}{\Delta x} \int_{x_{I-1}}^{b} \int_{c}^{d} V_{1}\left(\mathbf{x}, t^{n+1}\right) \eta\left(\mathbf{x}, t^{n}\right) \xi\left(b, y, t^{n+1}\right) d \mathbf{x} \\
- & \frac{\Delta t}{\Delta x} \int_{x_{1}}^{x_{2}} \int_{c}^{d} V_{1}\left(\mathbf{x}, t^{n+1}\right) \eta\left(\mathbf{x}, t^{n}\right) \xi\left(x_{1}, y, t^{n+1}\right) d \mathbf{x} \\
& -\frac{\Delta t}{\Delta x} \sum_{i=2}^{I-1} \int_{c}^{d} \int_{x_{i-1}}^{x_{i}}\left[V_{1}\left(x+\Delta x, y, t^{n+1}\right)-V_{1}\left(\mathbf{x}, t^{n+1}\right) d z\right] \\
& \eta\left(\mathbf{x}, t^{n}\right) \xi\left(x_{i}, y, t^{n+1}\right) d \mathbf{x} \\
& -\frac{\Delta t}{\Delta x} \sum_{i=2}^{I-1} \int_{c}^{d} \int_{x_{i-1}}^{x_{i}}\left[\eta\left(x+\Delta x, y, t^{n}\right)-\eta\left(\mathbf{x}, t^{n}\right)\right] \\
& V_{1}\left(x+\Delta x, y, t^{n+1}\right) \xi\left(x_{i}, y, t^{n+1}\right) d \mathbf{x} .
\end{aligned}
$$

Using (5.6), we bound the third term on the right-hand side of (7.6) by

$$
\left|\frac{\Delta t}{\Delta x} \sum_{i=2}^{I-1} \int_{c}^{d} \int_{x_{i-1}}^{x_{i}}\left[V_{1}\left(x+\Delta x, y, t^{n+1}\right)-V_{1}\left(\mathbf{x}, t^{n+1}\right)\right] \eta\left(\mathbf{x}, t^{n}\right) \xi\left(x_{i}, y, t^{n+1}\right) d \mathbf{x}\right|
$$

$$
\begin{aligned}
& \leq M \Delta t \sum_{i=2}^{I-1} \int_{c}^{d} \int_{x_{i-1}}^{x_{i}}\left|\eta\left(\mathbf{x}, t^{n}\right) \xi\left(x_{i}, y, t^{n+1}\right)\right| d \mathbf{x} \\
& \leq M \Delta t\left\|\xi\left(\mathbf{x}, t^{n+1}\right)\right\|_{L^{2}(\Omega)}\left\|\eta\left(\mathbf{x}, t^{n}\right)\right\|_{L^{2}(\Omega)} \\
& \leq M \Delta t\left\|\xi\left(\mathbf{x}, t^{n+1}\right)\right\|_{L^{2}(\Omega)}^{2}+M \Delta t h^{4}\left\|u\left(\mathbf{x}, t^{n}\right)\right\|_{H^{2}(\Omega)}^{2} .
\end{aligned}
$$

Introducing $\psi(x, y, t)$ by

$$
\psi(x, y, t):=u(x+\Delta x, y, t)-u(x, y, t)=\int_{0}^{\Delta x} u_{\alpha}(\alpha+x, y, t) d \alpha
$$

we have

$$
\begin{aligned}
& \eta\left(x+\Delta x, y, t^{n}\right)-\eta\left(x, y, t^{n}\right) \\
& \quad=\left(\Pi^{\mathrm{I}}-\mathrm{I}\right) u\left(x+\Delta x, y, t^{n}\right)-\left(\Pi^{\mathrm{I}}-\mathrm{I}\right) u\left(x, y, t^{n}\right)=\left(\Pi^{\mathrm{I}}-\mathrm{I}\right) \psi\left(x, y, t^{n}\right) .
\end{aligned}
$$

We combine the preceding estimate with (3.2) to obtain

$$
\begin{aligned}
& \sum_{i=1}^{I-1} \int_{c}^{d} \int_{x_{i-1}}^{x_{i}}\left[\eta\left(x+\Delta x, y, t^{n}\right)-\eta\left(\mathbf{x}, t^{n}\right)\right]^{2} d \mathbf{x} \\
& (7.8)=\sum_{j=1}^{J} \sum_{i=1}^{I-1} \int_{y_{j-1}}^{y_{j}} \int_{x_{i-1}}^{x_{i}}\left[\left.\left(\Pi^{\mathrm{I}}-\mathrm{I}\right) \psi\left(\mathbf{x}, t^{n}\right)\right|_{\left[x_{i-1}, x_{i}\right] \times\left[y_{j-1}, y_{j}\right]}\right]^{2} d \mathbf{x} \\
& =\left\|\left(\Pi^{\mathrm{I}}-\mathrm{I}\right) \psi\left(\mathbf{x}, t^{n}\right)\right\|_{L^{2}(\Omega)}^{2} \leq M h^{4}\left\|\psi\left(\mathbf{x}, t^{n}\right)\right\|_{H^{2}(\Omega)}^{2} \leq M h^{6}\left\|u\left(\mathbf{x}, t^{n}\right)\right\|_{H^{3}(\Omega)}^{2} .
\end{aligned}
$$


Using (7.8), we bound the last term on the right-hand side of (7.6) by

$$
\begin{aligned}
& \left|\frac{\Delta t}{\Delta x} \sum_{i=2}^{I-1} \int_{c}^{d} \int_{x_{i-1}}^{x_{i}}\left[\eta\left(x+\Delta x, y, t^{n}\right)-\eta\left(\mathbf{x}, t^{n}\right)\right] V_{1}\left(x+\Delta x, y, t^{n+1}\right) \xi\left(x_{i}, y, t^{n+1}\right) d \mathbf{x}\right| \\
& \quad \leq \frac{M \Delta t}{\Delta x}\left[\sum_{i=2}^{I-1} \int_{c}^{d} \int_{x_{i-1}}^{x_{i}}\left[\eta\left(x+\Delta x, y, t^{n}\right)-\eta\left(x, y, t^{n}\right)\right]^{2} d x d y\right]^{1 / 2}\left\|\xi\left(\mathbf{x}, t^{n+1}\right)\right\|_{L^{2}(\Omega)} \\
& \quad \leq M \Delta t h^{2}\left\|\xi\left(\mathbf{x}, t^{n+1}\right)\right\|_{L^{2}(\Omega)}\left\|u\left(\mathbf{x}, t^{n}\right)\right\|_{H^{3}(\Omega)} \\
& \quad \leq M \Delta t\left\|\xi\left(\mathbf{x}, t^{n+1}\right)\right\|_{L^{2}(\Omega)}^{2}+M \Delta t h^{4}\left\|u\left(\mathbf{x}, t^{n}\right)\right\|_{H^{3}(\Omega)}^{2},
\end{aligned}
$$

where at the first " $\leq$ " sign, we have used (5.6).

For $C r<1$, the last equality in (3.13) implies $\xi(\mathbf{x}, t)=\xi\left(\mathbf{x}, t^{n+1}\right)$ for $t \in\left[t^{n}, t^{n+1}\right]$. Hence, we bound the first term on the right-hand side of (7.6) by

$$
\begin{aligned}
& \left|\frac{\Delta t}{\Delta x} \int_{x_{I-1}}^{b} \int_{c}^{d} V_{1}\left(\mathbf{x}, t^{n+1}\right) \eta\left(\mathbf{x}, t^{n}\right) \xi\left(b, y, t^{n+1}\right) d \mathbf{x}\right| \\
& \quad \leq \frac{M \Delta t}{\Delta x}\left\|\eta\left(\mathbf{x}, t^{n}\right)\right\|_{L^{2}\left(\left[x_{I-1}, b\right] \times[c, d]\right)}\left[\int_{c}^{d} \int_{x_{I-1}}^{b} V_{1}\left(b, y, t^{n+1}\right) \xi^{2}\left(b, y, t^{n+1}\right) d \mathbf{x}\right]^{1 / 2} \\
& \quad \leq \frac{M(\Delta t)^{1 / 2}}{(\Delta x)^{1 / 2}} h^{2}\left\|u\left(\mathbf{x}, t^{n}\right)\right\|_{H^{2}\left(\left[x_{I-1}, b\right] \times[c, d]\right)}\left[\int_{t^{n}}^{t^{n+1}} \int_{c}^{d} \mathbf{v} \cdot \mathbf{n} \xi^{2}(\mathbf{x}, t) d S\right]^{1 / 2} \\
& \quad \leq \varepsilon \int_{t^{n}}^{t^{n+1}} \int_{c}^{d} \mathbf{v} \cdot \mathbf{n} \xi^{2}(\mathbf{x}, t) d S+M \Delta t h^{4}\left\|u\left(\mathbf{x}, t^{n}\right)\right\|_{H^{3}(\Omega)}^{2},
\end{aligned}
$$

where at the last step we have used (6.9).

Similarly, the second term on the right-hand side of (7.6) is bounded by

$$
\left|\frac{\Delta t}{\Delta x} \int_{x_{1}}^{x_{2}} \int_{c}^{d} V_{1}\left(\mathbf{x}, t^{n+1}\right) \eta\left(\mathbf{x}, t^{n}\right) \xi\left(x_{1}, y, t^{n+1}\right) d \mathbf{x}\right|
$$

$$
\begin{aligned}
& \leq \frac{M \Delta t}{\Delta x}\left\|\eta\left(\mathbf{x}, t^{n}\right)\right\|_{L^{2}\left(\left[x_{1}, x_{2}\right] \times[c, d]\right)}\left[\Delta x \int_{c}^{d}\left|V_{1}\left(x_{1}, y, t^{n+1}\right)\right| \xi^{2}\left(x_{1}, y, t^{n+1}\right) d y\right]^{1 / 2} \\
& \leq \frac{M(\Delta t)^{1 / 2}}{(\Delta x)^{1 / 2}} h^{2}\left\|u\left(\mathbf{x}, t^{n}\right)\right\|_{H^{2}\left(\left[x_{1}, x_{2}\right] \times[c, d]\right)}\left[\Delta t \int_{c}^{d}\left|V_{1}\left(x_{1}, y, t\right)\right| \xi^{2}\left(x_{1}, y, t\right) d y\right]^{1 / 2} \\
& \leq \varepsilon \Delta x \int_{c}^{d} K^{(5)}\left(x_{1}, y\right) \xi^{2}\left(x_{1}, y, t\right) d y+M \Delta t h^{4}\left\|u\left(\mathbf{x}, t^{n}\right)\right\|_{H^{3}(\Omega)}^{2}
\end{aligned}
$$

where at the last step we have used (6.9) again.

Combining (7.3)-(7.9), we have bounded the first term on the right-hand side of (7.2). By symmetry, we can bound the second term on the right-hand side of (7.2) in the same way. Thus, we have proven Lemma 3.

8. Proof of Lemma 4. In this section we derive an optimal-order estimate for the local truncation error term $E(\hat{\xi})$ defined by $(2.16)$.

Lemma 4. Assume $u(\mathbf{x}, t) \in L^{\infty}\left(0, T ; H^{1}(\Omega)\right)$. Then the following estimate holds:

$$
|E(\hat{\xi})| \leq \varepsilon \int_{S_{n}^{(O)}} \mathbf{v} \cdot \mathbf{n} \xi^{2}(\mathbf{x}, t) d S+\varepsilon \int_{\Omega^{(I)}\left(t^{n+1}\right) \cap\left(\left[x_{1}, b\right] \times\left[y_{1}, d\right]\right)} \xi^{2}\left(\mathbf{x}, t^{n+1}\right) d \mathbf{x}
$$




$$
\begin{aligned}
& +\varepsilon \Delta x \int_{y_{1}}^{d} K^{(5)}\left(x_{1}, y\right) \xi^{2}\left(x_{1}, y, t^{n+1}\right) d y+\varepsilon \Delta y \int_{x_{1}}^{b} K^{(6)}\left(x, y_{1}\right) \xi^{2}\left(x, y_{1}, t^{n+1}\right) d x \\
& +\varepsilon \Delta x \Delta y K^{(5)}\left(\mathbf{x}_{1,1}\right) K^{(6)}\left(\mathbf{x}_{1,1}\right) \xi^{2}\left(\mathbf{x}_{1,1}, t^{n+1}\right)+M \Delta t\left\|\xi\left(\mathbf{x}, t^{n+1}\right)\right\|_{L^{2}(\Omega)}^{2} \\
& +M \Delta t\|\xi(\mathbf{x}, t)\|_{L^{2}\left(S_{n}^{(O)}\right)}^{2}+M(\Delta t)^{3}\|u\|_{L^{\infty}\left(0, T ; H^{1}(\Omega)\right)}^{2} \\
& +M(\Delta t)^{2}\left[\|f\|_{L^{2}\left(t^{n}, t^{n+1} ; L^{2}(\Omega)\right)}^{2}+\left\|f_{\tau}\right\|_{L^{2}\left(t^{n}, t^{n+1} ; L^{2}(\Omega)\right)}^{2}\right]
\end{aligned}
$$

where $K^{(5)}(\mathbf{x})$ and $K^{(6)}(\mathbf{x})$ are defined in $(4.5)$ and $f_{\tau}$ is the derivative of $f$ along the (approximate) characteristics.

Proof. From (2.13), (2.14), and (2.16), the last term on the right-hand side of (4.1) is rewritten as

$$
\begin{aligned}
E(\hat{\xi})= & \int_{\Omega} \int_{t^{*}(\mathbf{x})}^{t^{n+1}}\left[f\left(\mathbf{r}\left(\theta ; \mathbf{x}, t^{n+1}\right), \theta\right) \operatorname{det} \mathbf{J}\left(\theta ; \mathbf{x}, t^{n+1}\right)-f\left(\mathbf{x}, t^{n+1}\right)\right] \hat{\xi}\left(\mathbf{x}, t^{n+1}\right) \\
& \times e^{-R\left(\mathbf{x}, t^{n+1}\right)\left(t^{n+1}-\theta\right)} d \theta d \mathbf{x} \\
+ & \int_{S_{n}^{(O)}} \int_{t^{*}(\mathbf{x}, t)}^{t} \mathbf{v} \cdot \mathbf{n}[f(\mathbf{r}(\theta ; \mathbf{x}, t), \theta) \operatorname{det} \mathbf{J}(\theta ; \mathbf{x}, t)-f(\mathbf{x}, t)] \xi(\mathbf{x}, t) \\
& \quad \times e^{-R(\mathbf{x}, t)(t-\theta)} d \theta d S \\
+ & \int_{t^{n}}^{t^{n+1}} \int_{\Omega} u(\mathbf{y}, \theta)\left[\hat{\xi}_{\theta}(\mathbf{y}, \theta)+\mathbf{v}(\mathbf{y}, \theta) \cdot \nabla \hat{\xi}(\mathbf{y}, \theta)-R(\mathbf{y}, \theta) \hat{\xi}(\mathbf{y}, \theta)\right] d \mathbf{y} d \theta
\end{aligned}
$$

where we have used $\mathbf{y}$ and $\theta$ as the dummy variables in the last integral and reserve $\mathbf{x}$ and $t$ for variables in $\Omega$ at time $t^{n+1}$ or the space-time outflow boundary $S_{n}^{(O)}$.

The first term on the right-hand side of $(8.1)$ is bounded by

$$
\begin{aligned}
& \mid \int_{\Omega} \int_{t^{*}(\mathbf{x})}^{t^{n+1}}\left[f\left(\mathbf{r}\left(\theta ; \mathbf{x}, t^{n+1}\right), \theta\right) \operatorname{det} \mathbf{J}\left(\theta ; \mathbf{x}, t^{n+1}\right)-f\left(\mathbf{x}, t^{n+1}\right)\right] \hat{\xi}\left(\mathbf{x}, t^{n+1}\right) \\
& \quad \times e^{-R\left(\mathbf{x}, t^{n+1}\right)\left(t^{n+1}-\theta\right)} d \theta d \mathbf{x} \mid \\
& \leq M \int_{\Omega} \int_{t^{*}(\mathbf{x})}^{t^{n+1}}\left|\operatorname{det} \mathbf{J}\left(\theta ; \mathbf{x}, t^{n+1}\right)-1\right|\left|f\left(\mathbf{r}\left(\theta ; \mathbf{x}, t^{n+1}\right), \theta\right) \hat{\xi}\left(\mathbf{x}, t^{n+1}\right)\right| d \theta d \mathbf{x} \\
& \quad+M \int_{\Omega} \int_{t^{*}(\mathbf{x})}^{t^{n+1}}\left|\int_{\theta}^{t^{n+1}} \frac{d f}{d \gamma}\left(\mathbf{r}\left(\gamma ; \mathbf{x}, t^{n+1}\right), \gamma\right) d \gamma\right|\left|\hat{\xi}\left(\mathbf{x}, t^{n+1}\right)\right| d \theta d \mathbf{x} \\
& \leq M(\Delta t)^{3 / 2}\left[\|f\|_{L^{2}\left(t^{n}, t^{n+1} ; L^{2}(\Omega)\right)}+\left\|f_{\tau}\right\|_{L^{2}\left(t^{n}, t^{n+1} ; L^{2}(\Omega)\right)}\right]\left\|\xi\left(\mathbf{x}, t^{n+1}\right)\right\|_{L^{2}(\Omega)} \\
& \leq M \Delta t\left\|\xi\left(\mathbf{x}, t^{n+1}\right)\right\|_{L^{2}(\Omega)}^{2}+C(\Delta t)^{2}\left[\|f\|_{L^{2}\left(t^{n}, t^{n+1} ; L^{2}(\Omega)\right)}^{2}+\left\|f_{\tau}\right\|_{L^{2}\left(t^{n}, t^{n+1} ; L^{2}(\Omega)\right)}^{2}\right]
\end{aligned}
$$

The second term on the right-hand side of (8.1) is bounded similarly:

$$
\begin{aligned}
& \left|\int_{S_{n}^{(O)}} \int_{t^{*}(\mathbf{x}, t)}^{t} \mathbf{v} \cdot \mathbf{n}[f(\mathbf{r}(\theta ; \mathbf{x}, t), \theta) \operatorname{det} \mathbf{J}(\theta ; \mathbf{x}, t)-f(\mathbf{x}, t)] \xi(\mathbf{x}, t) e^{-R(\mathbf{x}, t)(t-\theta)} d \theta d S\right| \\
& \quad \leq M \Delta t\|\xi(\mathbf{x}, t)\|_{L^{2}\left(S_{n}^{(O)}\right)}^{2}+C(\Delta t)^{2}\left[\|f\|_{L^{2}\left(t^{n}, t^{n+1} ; L^{2}(\Omega)\right)}^{2}+\left\|f_{\tau}\right\|_{L^{2}\left(t^{n}, t^{n+1} ; L^{2}(\Omega)\right)}^{2}\right] .
\end{aligned}
$$

At first glance, the last term on the right-hand side of (8.1) does not seem to contain any convergence factors. Nevertheless, the fact that the test functions $w$ 
defined by (2.8) satisfy the adjoint equation (2.4) approximately implies convergence rates. To see this, recall that the test functions satisfy

$$
\begin{array}{ll}
\xi_{\theta}\left(\mathbf{r}\left(\theta ; \mathbf{x}, t^{n+1}\right), \theta\right)+\mathbf{v}\left(\mathbf{x}, t^{n+1}\right) \cdot \nabla w\left(\mathbf{r}\left(\theta ; \mathbf{x}, t^{n+1}\right), \theta\right) & \\
- & R\left(\mathbf{x}, t^{n+1}\right) w\left(\mathbf{r}\left(\theta ; \mathbf{x}, t^{n+1}\right), \theta\right)=0, \quad \theta \in\left[t^{*}(\mathbf{x}), t^{n+1}\right], \quad \mathbf{x} \in \bar{\Omega} \\
\xi_{\theta}(\mathbf{r}(\theta ; \mathbf{x}, t), \theta)+\mathbf{v}(\mathbf{x}, t) \cdot \nabla w(\mathbf{r}(\theta ; \mathbf{x}, t), \theta) & \\
-R(\mathbf{x}, t) w(\mathbf{r}(\theta ; \mathbf{x}, t), \theta)=0, & \theta \in\left[t^{n}, t\right], \quad(\mathbf{x}, t) \in S_{n}^{(O)} .
\end{array}
$$

Hence, the last term on the right-hand side of (8.1) is rewritten as

$$
\begin{aligned}
\int_{t^{n}}^{t^{n+1}} & \int_{\Omega} u(\mathbf{y}, \theta)\left[\hat{\xi}_{\theta}(\mathbf{y}, \theta)+\mathbf{v}(\mathbf{y}, \theta) \cdot \nabla \hat{\xi}(\mathbf{y}, \theta)-R(\mathbf{y}, \theta) \hat{\xi}(\mathbf{y}, \theta)\right] d \mathbf{y} d \theta \\
= & \int_{t^{n}}^{t^{n+1}} \int_{\Omega \backslash \Omega(\theta)} u\left(\mathbf{r}\left(\theta ; \mathbf{x}, t^{n+1}\right), \theta\right)\left[\hat{\xi}_{\theta}+\mathbf{v} \cdot \nabla \hat{\xi}-R \hat{\xi}\right]\left(\mathbf{r}\left(\theta ; \mathbf{x}, t^{n+1}\right), \theta\right) d \mathbf{r} d \theta \\
(8.3)+ & \int_{t^{n}}^{t^{n+1}} \int_{\Omega(\theta)} u(\mathbf{r}(\theta ; \mathbf{x}, t), \theta)\left[\xi_{\theta}+\mathbf{v} \cdot \nabla \xi-R \xi\right](\mathbf{r}(\theta ; \mathbf{x}, t), \theta) d \mathbf{r} d \theta \\
= & -\int_{t^{n}}^{t^{n+1}} \int_{\Omega \backslash \Omega(\theta)}\left[\mathbf{v}\left(\mathbf{x}, t^{n+1}\right)-\mathbf{v}\left(\mathbf{r}\left(\theta ; \mathbf{x}, t^{n+1}\right), \theta\right)\right] \cdot(u \nabla \hat{\xi})\left(\mathbf{r}\left(\theta ; \mathbf{x}, t^{n+1}\right), \theta\right) d \mathbf{r} d \theta \\
& -\int_{t^{n}}^{t^{n+1}} \int_{\Omega(\theta)}[\mathbf{v}(\mathbf{x}, t)-\mathbf{v}(\mathbf{r}(\theta ; \mathbf{x}, t), \theta)] \cdot(u \nabla \xi)(\mathbf{r}(\theta ; \mathbf{x}, t), \theta) d \mathbf{r} d \theta \\
& +\int_{t^{n}}^{t^{n+1}} \int_{\Omega \backslash \Omega(\theta)}\left[R\left(\mathbf{x}, t^{n+1}\right)-R\left(\mathbf{r}\left(\theta ; \mathbf{x}, t^{n+1}\right), \theta\right)\right](u \hat{\xi})\left(\mathbf{r}\left(\theta ; \mathbf{x}, t^{n+1}\right), \theta\right) d \mathbf{r} d \theta \\
& +\int_{t^{n}}^{t^{n+1}} \int_{\Omega(\theta)}[R(\mathbf{x}, t)-R(\mathbf{r}(\theta ; \mathbf{x}, t), \theta)](u \xi)(\mathbf{r}(\theta ; \mathbf{x}, t), \theta) d \mathbf{r} d \theta .
\end{aligned}
$$

The last two terms on the right-hand side of (8.3) are bounded by

$$
\begin{aligned}
& \mid \int_{t^{n}}^{t^{n+1}} \int_{\Omega \backslash \Omega(\theta)}\left[R\left(\mathbf{x}, t^{n+1}\right)-R\left(\mathbf{r}\left(\theta ; \mathbf{x}, t^{n+1}\right), \theta\right)\right](u \hat{\xi})\left(\mathbf{r}\left(\theta ; \mathbf{x}, t^{n+1}\right), \theta\right) d \mathbf{r} d \theta \\
& \quad+\int_{t^{n}}^{t^{n+1}} \int_{\Omega(\theta)}[R(\mathbf{x}, t)-R(\mathbf{r}(\theta ; \mathbf{x}, t), \theta)](u \xi)(\mathbf{r}(\theta ; \mathbf{x}, t), \theta) d \mathbf{r} d \theta \mid
\end{aligned}
$$

$\leq M \Delta t\left\{(\Delta t)^{1 / 2}\left\|\xi\left(\mathbf{x}, t^{n+1}\right)\right\|_{L^{2}(\Omega)}+\left[\int_{S_{n}^{(O)}} \mathbf{v} \cdot \mathbf{n} \xi^{2}(\mathbf{x}, t) d S\right]^{1 / 2}\right\}$

$$
\begin{aligned}
& \|u\|_{L^{2}\left(t^{n}, t^{n+1} ; L^{2}(\Omega)\right)} \\
\leq & \varepsilon \int_{S_{n}^{(O)}} \mathbf{v} \cdot \mathbf{n} \xi^{2}(\mathbf{x}, t) d S+M \Delta t\left\|\xi\left(\mathbf{x}, t^{n+1}\right)\right\|_{L^{2}(\Omega)}^{2} \\
& +M(\Delta t)^{2}\|u\|_{L^{2}\left(t^{n}, t^{n+1} ; L^{2}(\Omega)\right)}^{2} .
\end{aligned}
$$

Since we cannot hide the term $M \Delta t\left\|\nabla \xi\left(\mathbf{x}, t^{n+1}\right)\right\|_{L^{2}(\Omega)}^{2}$, we treat the first and second terms on the right-hand side of (8.3) in a different way. We obtain

$-\int_{t^{n}}^{t^{n+1}} \int_{\Omega \backslash \Omega(\theta)}\left[\mathbf{v}\left(\mathbf{x}, t^{n+1}\right)-\mathbf{v}\left(\mathbf{r}\left(\theta ; \mathbf{x}, t^{n+1}\right), \theta\right)\right] \cdot(u \nabla \hat{\xi})\left(\mathbf{r}\left(\theta ; \mathbf{x}, t^{n+1}\right), \theta\right) d \mathbf{r} d \theta$ 


$$
\begin{aligned}
& -\int_{t^{n}}^{t^{n+1}} \int_{\Omega(\theta)}[\mathbf{v}(\mathbf{x}, t)-\mathbf{v}(\mathbf{r}(\theta ; \mathbf{x}, t), \theta)] \cdot(u \nabla \xi)(\mathbf{r}(\theta ; \mathbf{x}, t), \theta) d \mathbf{r} d \theta \\
= & \int_{t^{n}}^{t^{n+1}} \int_{\Omega \backslash \Omega(\theta)} \nabla \cdot\left[\mathbf{v}\left(\mathbf{x}, t^{n+1}\right)-\mathbf{v}\left(\mathbf{r}\left(\theta ; \mathbf{x}, t^{n+1}\right), \theta\right)\right](u \hat{\xi})\left(\mathbf{r}\left(\theta ; \mathbf{x}, t^{n+1}\right), \theta\right) d \mathbf{r} d \theta \\
(8.5)+ & \int_{t^{n}}^{t^{n+1}} \int_{\Omega(\theta)} \nabla \cdot[\mathbf{v}(\mathbf{x}, t)-\mathbf{v}(\mathbf{r}(\theta ; \mathbf{x}, t), \theta)](u \xi)(\mathbf{r}(\theta ; \mathbf{x}, t), \theta) d \mathbf{r} d \theta \\
& +\int_{t^{n}}^{t^{n+1}} \int_{\Omega \backslash \Omega(\theta)}\left[\mathbf{v}\left(\mathbf{x}, t^{n+1}\right)-\mathbf{v}\left(\mathbf{r}\left(\theta ; \mathbf{x}, t^{n+1}\right), \theta\right)\right] \cdot(\nabla u \hat{\xi})\left(\mathbf{r}\left(\theta ; \mathbf{x}, t^{n+1}\right), \theta\right) d \mathbf{r} d \theta \\
& +\int_{t^{n}}^{t^{n+1}} \int_{\Omega(\theta)}[\mathbf{v}(\mathbf{x}, t)-\mathbf{v}(\mathbf{r}(\theta ; \mathbf{x}, t), \theta)] \cdot(\nabla u \xi)(\mathbf{r}(\theta ; \mathbf{x}, t), \theta) d \mathbf{r} d \theta \\
& -\int_{t^{n}}^{t^{n+1}} \int_{\partial(\Omega \backslash \Omega(\theta))}\left[\mathbf{v}\left(\mathbf{x}, t^{n+1}\right)-\mathbf{v}\left(\mathbf{r}\left(\theta ; \mathbf{x}, t^{n+1}\right), \theta\right)\right] \cdot \mathbf{n}(u \hat{\xi})\left(\mathbf{r}\left(\theta ; \mathbf{x}, t^{n+1}\right), \theta\right) d s d \theta \\
& -\int_{t^{n}}^{t^{n+1}} \int_{\partial(\Omega(\theta))}[\mathbf{v}(\mathbf{x}, t)-\mathbf{v}(\mathbf{r}(\theta ; \mathbf{x}, t), \theta)] \cdot \mathbf{n}(u \xi)(\mathbf{r}(\theta ; \mathbf{x}, t), \theta) d s d \theta .
\end{aligned}
$$

The first through fourth terms on the right-hand side of the previous equation are bounded as in (8.4):

$$
\begin{aligned}
& \mid \int_{t^{n}}^{t^{n+1}} \int_{\Omega \backslash \Omega(\theta)} \nabla \cdot\left[\mathbf{v}\left(\mathbf{x}, t^{n+1}\right)-\mathbf{v}\left(\mathbf{r}\left(\theta ; \mathbf{x}, t^{n+1}\right), \theta\right)\right](u \hat{\xi})\left(\mathbf{r}\left(\theta ; \mathbf{x}, t^{n+1}\right), \theta\right) d \mathbf{r} d \theta \\
& \quad+\int_{t^{n}}^{t^{n+1}} \int_{\Omega(\theta)} \nabla \cdot[\mathbf{v}(\mathbf{x}, t)-\mathbf{v}(\mathbf{r}(\theta ; \mathbf{x}, t), \theta)](u \hat{\xi})(\mathbf{r}(\theta ; \mathbf{x}, t), \theta) d \mathbf{r} d \theta \\
& \quad+\int_{t^{n}}^{t^{n+1}} \int_{\Omega \backslash \Omega(\theta)}\left[\mathbf{v}\left(\mathbf{x}, t^{n+1}\right)-\mathbf{v}\left(\mathbf{r}\left(\theta ; \mathbf{x}, t^{n+1}\right), \theta\right)\right] \cdot(\nabla u \hat{\xi})\left(\mathbf{r}\left(\theta ; \mathbf{x}, t^{n+1}\right), \theta\right) d \mathbf{r} d \theta \\
& \quad+\int_{t^{n}}^{t^{n+1}} \int_{\Omega(\theta)}[\mathbf{v}(\mathbf{x}, t)-\mathbf{v}(\mathbf{r}(\theta ; \mathbf{x}, t), \theta)] \cdot(\nabla u \hat{\xi})(\mathbf{r}(\theta ; \mathbf{x}, t), \theta) d \mathbf{r} d \theta \mid \\
& \leq \varepsilon \int_{S_{n}^{(O)}} \mathbf{v} \cdot \mathbf{n} \xi^{2}(\mathbf{x}, t) d S+M \Delta t\left\|\xi\left(\mathbf{x}, t^{n+1}\right)\right\|_{L^{2}(\Omega)}^{2}+M(\Delta t)^{2}\|u\|_{L^{2}\left(t^{n}, t^{n+1} ; H^{1}(\Omega)\right)}
\end{aligned}
$$

Notice that the two integrals on $\partial(\Omega \backslash \Omega(\theta))-S_{n}^{(I)}$ and $\partial(\Omega(\theta))-S_{n}^{(O)}$ cancel each other out. We bound the last two terms on the right-hand side of (8.5) by

$$
\begin{aligned}
& \mid \int_{t^{n}}^{t^{n+1}} \int_{\partial(\Omega \backslash \Omega(\theta))}\left[\mathbf{v}\left(\mathbf{x}, t^{n+1}\right)-\mathbf{v}\left(\mathbf{r}\left(\theta ; \mathbf{x}, t^{n+1}\right), \theta\right)\right] \cdot \mathbf{n}(u \hat{\xi})\left(\mathbf{r}\left(\theta ; \mathbf{x}, t^{n+1}\right), \theta\right) d s d \theta \\
& \quad+\int_{t^{n}}^{t^{n+1}} \int_{\partial(\Omega(\theta))}[\mathbf{v}(\mathbf{x}, t)-\mathbf{v}(\mathbf{r}(\theta ; \mathbf{x}, t), \theta)] \cdot \mathbf{n}(u \xi)(\mathbf{r}(\theta ; \mathbf{x}, t), \theta) d s d \theta \mid \\
& \leq M \Delta t \int_{S_{n}^{(I)}}\left|u(\mathbf{x}, t) \xi\left(\mathbf{x}^{+}, t^{+}\right)\right| d S+M \Delta t \int_{S_{n}^{(O)}}|u(\mathbf{x}, t) \xi(\mathbf{x}, t)| d S \\
& \leq \varepsilon \int_{S_{n}^{(O)}} \mathbf{v} \cdot \mathbf{n} \xi^{2}(\mathbf{x}, t) d S+\varepsilon \int_{\Omega^{(I)}\left(t^{n+1}\right)} \xi^{2}\left(\mathbf{x}, t^{n+1}\right) d \mathbf{x} \\
& \quad+M(\Delta t)^{3}\|u\|_{L^{\infty}\left(0, T ; H^{1}(\Omega)\right)}^{2}
\end{aligned}
$$




$$
\begin{aligned}
& \leq \varepsilon \int_{S_{n}^{(O)}} \mathbf{v} \cdot \mathbf{n} \xi^{2}(\mathbf{x}, t) d S+\varepsilon \int_{\Omega^{(I)}\left(t^{n+1}\right) \cap\left(\left[x_{1}, b\right] \times\left[y_{1}, d\right]\right)} \xi^{2}\left(\mathbf{x}, t^{n+1}\right) d \mathbf{x} \\
& +\varepsilon \Delta x \int_{y_{1}}^{d} K^{(5)}\left(x_{1}, y\right) \xi^{2}\left(x_{1}, y, t^{n+1}\right) d y+\varepsilon \Delta y \int_{x_{1}}^{b} K^{(6)}\left(x, y_{1}\right) \xi^{2}\left(x, y_{1}, t^{n+1}\right) d x \\
& +\varepsilon \Delta x \Delta y K^{(5)}\left(\mathbf{x}_{1,1}\right) K^{(6)}\left(\mathbf{x}_{1,1}\right) \xi^{2}\left(\mathbf{x}_{1,1}, t^{n+1}\right)+M \Delta t\left\|\xi\left(\mathbf{x}, t^{n+1}\right)\right\|_{L^{2}(\Omega)}^{2} \\
& +M(\Delta t)^{3}\|u\|_{L^{\infty}\left(0, T ; H^{1}(\Omega)\right)}^{2}
\end{aligned}
$$

Combining (8.1)-(8.6), we have proven Lemma 4.

9. Numerical experiments. In this section we perform numerical experiments to verify the theoretically proven optimal-order $L^{2}$ convergence rates. The test example is the transport of a two-dimensional rotating Gaussian pulse. The spatial domain is $\Omega=(-0.5,0.5) \times(-0.5,0.5)$, the rotating field is imposed as $V_{1}(x, y)=-4 y$, and $V_{2}(x, y)=4 x$. The reaction coefficient is $R=0.4 \cos (2 t)$. The time interval is $[0, T]=[0, \pi / 2]$, which is the time period required for one complete rotation. The initial condition $u_{0}(x, y)$ is given by

$$
u_{0}(x, y):=\exp \left(-\frac{\left(x-x_{c}\right)^{2}+\left(y-y_{c}\right)^{2}}{2 \sigma^{2}}\right)
$$

where $x_{c}, y_{c}$, and $\sigma$ are the centered and standard deviations, respectively. The corresponding analytical solution for (2.1) with $f=0$ is given by

$$
u(x, y, t)=\exp \left(-\frac{\left(\bar{x}-x_{c}\right)^{2}+\left(\bar{y}-y_{c}\right)^{2}}{2 \sigma^{2}}-0.2 \sin (2 t)\right)
$$

where $\bar{x}=x \cos (4 t)+y \sin (4 t)$ and $\bar{y}=-x \sin (4 t)+y \cos (4 t)$.

In the numerical experiments, the data are chosen as follows: $x_{c}=-0.25, y_{c}=0$, $\sigma=0.0447$, which gives $2 \sigma^{2}=0.0040$. This problem provides an example for a homogeneous two-dimensional reactive transport equation with a variable velocity field and a known analytical solution. This example has been widely used to test for numerical artifacts of different schemes, such as numerical stability and numerical dispersion, spurious oscillations, and phase errors. Our previous results $[28,29]$ showed that the ELLAM scheme yields accurate numerical solutions even though large time steps are used. Moreover, the numerical solutions are free of numerical diffusion, spurious oscillation, and phase errors.

In this section we use a linear regression to fit the convergence rates and the associated constants in the error estimates

$$
\|U(\mathbf{x}, T)-u(\mathbf{x}, T)\|_{L^{p}(\Omega)} \leq M_{\alpha} h^{\alpha}+M_{\beta}(\Delta t)^{\beta}, \quad p=1,2 .
$$

We perform two kinds of computations. The first tests the spatial convergence rate of the ELLAM scheme (2.21), where we fix a small time step $\Delta t$ and compute the constant $M_{\alpha}$ and the rate $\alpha$ with respect to $h$; the other tests the temporal convergence rate, where we choose a small grid size $h$ and calculate the constant $M_{\beta}$ and the rate $\beta$ with respect to $\Delta t$. The results are presented in Tables 9.1 and 9.2, fitting the pairs $\left(M_{\alpha}, \alpha\right)$ and $\left(M_{\beta}, \beta\right)$, respectively. For simplicity in these tables we present only the errors $\|U(\mathbf{x}, T)-u(\mathbf{x}, T)\|_{L^{p}(\Omega)}$ instead of the uniform-in-time error $\|U(\mathbf{x}, T)-u(\mathbf{x}, T)\|_{\hat{L}^{\infty}\left(0, T ; L^{p}(\Omega)\right)}$, since the latter is expected to be reached by $\|U(\mathbf{x}, T)-u(\mathbf{x}, T)\|_{L^{p}(\Omega)}$ at the final time $t=T$. The results show that the ELLAM 
TABLE 9.1

Convergence rates in space, test for $M_{\alpha}$ and $\alpha$.

\begin{tabular}{|c|c|c|c|}
\hline$\Delta t$ & $\Delta x=\Delta y$ & $\|U(\mathbf{x}, T)-u(\mathbf{x}, T)\|_{L^{2}(\Omega)}$ & $\|U(\mathbf{x}, T)-u(\mathbf{x}, T)\|_{L^{1}(\Omega)}$ \\
\hline$\pi / 60$ & $1 / 40$ & $4.4422 \times 10^{-3}$ & $7.3693 \times 10^{-4}$ \\
\hline$\pi / 60$ & $1 / 48$ & $2.6739 \times 10^{-3}$ & $4.5730 \times 10^{-4}$ \\
\hline$\pi / 60$ & $1 / 56$ & $1.8331 \times 10^{-3}$ & $3.2512 \times 10^{-4}$ \\
\hline$\pi / 60$ & $1 / 64$ & $1.2876 \times 10^{-3}$ & $2.4048 \times 10^{-4}$ \\
\hline & & $M_{\alpha}=69.12, \alpha=2.62$ & $M_{\alpha}=4.57, \alpha=2.37$ \\
\hline
\end{tabular}

TABLE 9.2

Convergence rates in time, test for $M_{\beta}$ and $\beta$.

\begin{tabular}{|c|c|c|c|}
\hline$\Delta t$ & $\Delta x=\Delta y$ & $\|U(\mathbf{x}, T)-u(\mathbf{x}, T)\|_{L^{2}(\Omega)}$ & $\|U(\mathbf{x}, T)-u(\mathbf{x}, T)\|_{L^{1}(\Omega)}$ \\
\hline$\pi / 28$ & $1 / 64$ & $2.1875 \times 10^{-2}$ & $4.1510 \times 10^{-3}$ \\
\hline$\pi / 32$ & $1 / 64$ & $1.8225 \times 10^{-2}$ & $3.6232 \times 10^{-3}$ \\
\hline$\pi / 36$ & $1 / 64$ & $1.7047 \times 10^{-2}$ & $3.2190 \times 10^{-3}$ \\
\hline$\pi / 40$ & $1 / 64$ & $1.4469 \times 10^{-2}$ & $2.8931 \times 10^{-3}$ \\
\hline & & $M_{\beta}=0.85, \beta=1.10$ & $M_{\beta}=0.12, \beta=1.01$ \\
\hline
\end{tabular}

scheme (2.21) possesses second-order accuracy in space and first-order accuracy in time as predicted by Theorem 1 in section 4 . Moreover, we notice that in the numerical experiments $M_{\beta}$ is much smaller than $M_{\alpha}$. This reflects the fact that the solutions of (2.1) are not smooth in space but are much smoother along characteristics, and it justifies the use of the Lagrangian coordinates in the ELLAM schemes.

\section{REFERENCES}

[1] J. W. Barrett And K. W. Morton, Approximate symmetrization and Petrov-Galerkin methods for diffusion-convection problems, Comput. Methods Appl. Mech. Engrg., 45 (1984), pp. 97-122.

[2] J. BEAR, Hydraulics of Groundwater, McGraw-Hill, New York, 1979.

[3] A. Brooks And T. J. R. Hughes, Streamline upwind Petrov-Galerkin formulations for convection dominated flows with particular emphasis on the incompressible Navier-Stokes equations, Comput. Methods Appl. Mech. Engrg., 32 (1982), pp. 199-259.

[4] M. A. Celia, T. F. Russell, I. Herrera, And R. E. Ewing, An Eulerian-Lagrangian localized adjoint method for the advection-diffusion equation, Adv. in Water Resources, 13 (1990), pp. 187-206.

[5] I. Christie, D. F. Griffiths, A. R. Mitchell, And O. C. Zienkiewicz, Finite element methods for second order differential equations with significant first derivatives, Internat. J. Numer. Methods Engrg., 10 (1976), pp. 1389-1396.

[6] P. G. Ciarlet, The Finite Element Method for Elliptic Problems, North-Holland, Amsterdam, 1978.

[7] P. Colella, A direct Eulerian MUSCL scheme for gas dynamics, SIAM J. Sci. Statist. Comput., 6 (1985), pp. 104-117.

$\rightarrow$ M. G. CRANDAll AND A. MAJDA, Monotone difference approximations for scalar conservation laws, Math. Comp., 34 (1980), pp. 1-21. 
[9] C. N. Dawson, T. F. Dupont, And M. F. WheEler, The rate of convergence of the modified method of characteristics for linear advection equation in one dimension, in Mathematics for Large Scale Computing, J. C. Diaz, ed., Marcel Dekker, New York, 1989, pp. 115-126.

[10] C. N. Dawson, T. F. Russell, And M. F. Wheeler, Some improved error estimates for the modified method of characteristics, SIAM J. Numer. Anal., 26 (1989), pp. 1487-1512.

[11] F.-J. Delvos AND W. SchempP, Boolean Methods in Interpolation and Approximation, Longman Scientific \& Technical, Harlow, UK, 1989.

$[1 \rightarrow$ J. Douglas, JR. AND T. F. Russell, Numerical methods for convection-dominated diffusion problems based on combining the method of characteristics with finite element or finite difference procedures, SIAM J. Numer. Anal., 19 (1982), pp. 871-885.

[13] R. E. EwIng, ED., The Mathematics of Reservoir Simulation, Frontiers Appl. Math. 1, SIAM, Philadelphia, 1984.

[14] R. E. EWING AND H. WANG, Eulerian-Lagrangian localized adjoint methods for linear advection or advection-reaction equations and their convergence analysis, Comput. Mech., 12 (1993), pp. 97-121.

[15] R. E. EWING AND H. WANG, An optimal-order error estimate for Eulerian-Lagrangian localized adjoint methods for variable-coefficient advection-reaction problems, SIAM J. Numer. Anal., 33 (1996), pp. 318-348.

[16] C. L. FARMER, A moving point method for arbitrary Peclet number multi-dimensional convection-diffusion equations, IMA J. Numer. Anal., 5 (1980), pp. 465-480.

[17] A. Harten, B. Engquist, S. Osher, And S. Chakravarthy, Uniformly high order accurate essentially nonoscillatory schemes, III, J. Comput. Phys., 71 (1987), pp. 231-241.

[18] C. Johnson, Numerical Solutions of Partial Differential Equations by the Finite Element Method, Cambridge University Press, Cambridge, 1987.

[19] C. Johnson, A. Szepessy, And P. Hansbo, On the convergence of shock-capturing streamline diffusion finite element methods for hyperbolic conservation laws, Math. Comp., 54 (1990), pp. 107-129.

[20] P. Lancaster and K. Šalkauskas, Curve and Surface Fitting, An Introduction, Academic Press, New York, 1986.

[21] R. J. LeVEque, Numerical Methods for Conservation Laws, Birkhäuser Verlag, Basel, 1992.

[22] K. W. Morton, A. Priestley, And E. Süli, Stability of the Lagrangian-Galerkin method with nonexact integration, RAIRO Modél. Math. Anal. Numér., 22 (1988), pp. 123-151.

[23] O. Pironneau, On the transport-diffusion algorithm and its application to the Navier-Stokes equations, Numer. Math., 38 (1982), pp. 309-332.

[24] C. SHU AND S. Osher, Efficient implementation of essentially non-oscillatory shock capturing schemes, J. Comput. Phys., 77 (1988), pp. 439-471.

$[\because \rightarrow$ P. K. SwEBY, High resolution schemes using flux limiters for hyperbolic conservation laws, SIAM J. Numer. Anal., 21 (1984), pp. 995-1011.

[26] B. VAN LEER, On the relation between the upwind-differencing schemes of Godunov, EngquistOsher and Roe, SIAM J. Sci. Statist. Comput., 5 (1984), pp. 1-20.

[27] E. VARoglu AND W. D. L. Finn, Finite elements incorporating characteristics for onedimensional diffusion-convection equation, J. Comput. Phys., 34 (1980), pp. 371-389.

[28] H. Wang, M. Al-Lawatia, and S. A. Telyakovskiy, A Runge-Kutta characteristic method for first-order linear hyperbolic equations, Numer. Methods Partial Differential Equations, 13 (1997), pp. 617-661.

[29] H. Wang, R. E. Ewing, G. Qin, S. L. Lyons, M. Al-Lawatia, And S. Man, A family of Eulerian-Lagrangian localized adjoint methods for multi-dimensional advection-reaction equations, J. Comput. Phys., 152 (1999), pp. 120-163. 